\title{
Squeezed Coherent States in Non-Unitary Approach and Relation to Sub- and Super-Poissonian Statistics
}

\author{
Alfred Wünsche \\ Institut für Physik, Humboldt-Universität, Newtonstr, Berlin, Germany \\ Email: alfred.wuensche@physik.hu-berlin.de
}

How to cite this paper: Wünsche, A. (2017) Squeezed Coherent States in Non-Unitary Approach and Relation to Sub- and Super-Poissonian Statistics. Advances in Pure Mathematics, 7, 706-757.

https://doi.org/10.4236/apm.2017.712044

Received: November 13, 2017

Accepted: December 26, 2017

Published: December 29, 2017

Copyright $\odot 2017$ by author and Scientific Research Publishing Inc. This work is licensed under the Creative Commons Attribution International License (CC BY 4.0).

http://creativecommons.org/licenses/by/4.0/

\section{(c) (i) Open Access}

\begin{abstract}
After developing the concept of displaced squeezed vacuum states in the nonunitary approach and establishing the connection to the unitary approach we calculate their quasiprobabilities and expectation values $a^{\dagger k} a$ in general form. Then we consider the displacement of the squeezed vacuum states and calculate their photon statistics and their quasiprobabilities. The expectation values of the displaced states are related to the expectation values of the undisplaced states and are calculated for some simplest cases which are sufficient to discuss their categorization as sub-Poissonian and super-Poissonian statistics. A large set of these states do not belong to sub- or to super-Poissonian states but are also not Poissonian states. We illustrate in examples their photon distributions. This shows that the notions of sub- and of super-Poissonian statistics and their use for the definition of nonclassicality of states are problematic. In Appendix A we present the most important relations for $S U(1,1)$ treatment of squeezing and the disentanglement of their operators. Some initial members of sequences of expectation values for squeezed vacuum states are collected in Appendix E.
\end{abstract}

\section{Keywords}

$S U(1,1)$ Group of Squeezing and Rotation, Wigner Quasiprobability, Unitary Approach to Squeezing, Nonclassical States, Uncertainty Matrix, Distance of States, Jacobi, Ultraspherical, Legendre and Hermite Polynomials, Poisson Statistics

\section{Introduction}

Besides the number states $|n\rangle$ and the coherent states $|\alpha\rangle$ the squeezed 
coherent states or, what is the same, the displaced squeezed vacuum states belong to the most interesting states in quantum optics for which, practically, all interesting parameters and quasiprobabilities may be calculated in closed exact way. The coherent states are the vacuum states $|n=0\rangle$ displaced by a complex parameter $\beta$ in the phase space (for one mode). The higher number states $|n\rangle$ with $n \neq 0$ are the discrete excitations of the ground state $|n=0\rangle$ of a harmonic oscillator and they also can be displaced and squeezed but this we do not consider in present article. All minimum uncertainty states belong to the squeezed coherent states and therefore some aspects of these states were already considered in the early years of the development of quantum mechanics although not under this name, for example, by Schrödinger [1], Pauli [2] and Louisell [3]. The name "squeezed states" appeared in the eighties by Walls [4] and others and numerous articles and reviews are published since this time, e.g., [5]-[14] and, e.g., [15] [16] [17] [18] [19].

In the narrow sense the squeezing operations form together with rotations in a plane (the two-dimensional phase plane) the Lie group $S U(1,1)$ with 3 real parameters. This Lie group possesses different realizations in quantum optics of a single mode and also a basic nontrivial realization in a two-mode system. We will deal with in this article a single mode where the basic operators of the Lie group $S U(1,1)$ are realized by quadratic combinations of the annihilation and creation operators $\left(a, a^{\dagger}\right)$ of this mode but in Appendix A we represent in detail the basic relations for $S U(1,1)$. Besides this, the Lie group $S U(1,1)$ may find application within a single mode also for the treatment of phase states and as mentioned possesses a basic realization in a two-mode system (e.g., [15]). Dynamical squeezing appears if the Hamiltonian or Liouvillean of a process is described by quadratic combinations of annihilation and creation operators.

The main purpose of this paper is the representation of the formalism of $S U(1,1)$ squeezing in two approaches concerning the complex squeezing parameter which we call non-unitary and unitary approach and the calculation of expectation values and of the basic quasiprobabilities for squeezed vacuum and squeezed coherent states. The squeezed coherent states are well appropriate to demonstrate some problems of the distinction of sub- and super-Poissonian photon statistics because the whole set of these states can be not assigned to only one of these two kinds of statistics and it requires substantial efforts to find out to which of these statistics it belongs in a special case. The cases when they are neither sub- nor super-Poissonian statistics may be very far from a Poisson statistics that can be seen by the distance parameter. This shows in an example of nonclassical states the problems of classification of statistics in quantum optics in this way and is discussed in Section 11.

\section{Squeezed Vacuum States in Non-Unitary Approach and Their Photon Statistics}

In this section we begin with the discussion of squeezed vacuum states in the non-unitary approach. For their definition we apply the non-unitary operator 
$\exp \left(-\frac{\zeta}{2} a^{\dagger 2}\right)$ with $\zeta$ as a complex parameter $(|\zeta|<1)$ onto the vacuum state $|0\rangle$. As usual, $\left(a, a^{\dagger}\right)$ denote a pair of annihilation and creation operators of a single boson mode with the commutation relations $\left[a, a^{\dagger}\right] \equiv a a^{\dagger}-a^{\dagger} a=I$, ( $I$ unity operator) and they act onto the number states n) which are orthonormalized and complete

$$
\begin{array}{r}
a|n\rangle=\sqrt{n}|n-1\rangle, \quad a^{\dagger}|n\rangle=\sqrt{n+1}|n+1\rangle, \quad N|n\rangle \equiv a^{\dagger} a|n\rangle=n|n\rangle, \\
\langle m \mid n\rangle=\delta_{m, n}, \quad \sum_{n=0}^{\infty}|n\rangle\langle n|=I, \quad| n\rangle=\frac{a^{\dagger n}}{\sqrt{n !}}|0\rangle, \quad(n=0,1,2, \cdots) .
\end{array}
$$

Now, we define the squeezed vacuum states $|0, \zeta\rangle$ in the non-unitary approach by

$$
|0, \zeta\rangle \propto \exp \left(-\frac{\zeta}{2} a^{\dagger 2}\right)|0\rangle=\sum_{m=0}^{\infty} \frac{(-1)^{m}}{2^{m} m !} \zeta^{m} a^{\dagger 2 m}|0\rangle=\sum_{m=0}^{\infty} \frac{(-1)^{m} \sqrt{(2 m) !}}{2^{m} m !} \zeta^{m}|2 m\rangle,
$$

where the zero in the notation $|0, \zeta\rangle$ is arranged for the substitution by a complex displacement parameter $\beta$ in the later generalization to displaced vacuum states $|\beta, \zeta\rangle$ (see Figure 1 and from Section 7 on).

Since $\mathrm{e}^{-\frac{\zeta}{2} a^{+2}}$ is not a unitary operator the right-hand side of (2) is not normalized and using the Taylor series $\sum_{m=0}^{\infty} \frac{(2 m) !}{2^{2 m} m !^{2}} q^{m}=\frac{1}{\sqrt{1-q}}$ we find the normalization factor

$$
\begin{aligned}
& |0, \zeta\rangle=\left(1-\zeta \zeta^{*}\right)^{\frac{1}{4}} \exp \left(-\frac{\zeta}{2} a^{\dagger 2}\right)|0\rangle \\
& =\left(1-\zeta \zeta^{*}\right)^{\frac{1}{4}} \sum_{m=0}^{\infty} \frac{(-1)^{m} \sqrt{(2 m) !}}{2^{m} m !} \zeta^{m}|2 m\rangle,\langle 0, \zeta \mid 0, \zeta\rangle=1,|\zeta| \equiv \sqrt{\zeta \zeta^{*}}<1 .
\end{aligned}
$$

The complex parameter $\zeta$ is restricted in the non-unitary approach for normalizable states to $|\zeta|<1$ but can be continued to non-normalizable states for $|\zeta| \geq 1$. In the unitary approach (2) we apply a unitary operator $\exp \left(\frac{\zeta^{\prime *}}{2} a^{2}-\frac{\zeta^{\prime}}{2} a^{\dagger 2}\right)$ to the vacuum state $|0\rangle$ according to

$$
|0, \zeta\rangle \equiv \exp \left\{\frac{\operatorname{Arth}|\zeta|}{|\zeta|}\left(\frac{\zeta^{*}}{2} a^{2}-\frac{\zeta}{2} a^{\dagger 2}\right)\right\}|0\rangle \equiv \exp \left(\frac{\zeta^{\prime *}}{2} a^{2}-\frac{\zeta^{\prime}}{2} a^{\dagger 2}\right)|0\rangle \text {. }
$$

The connection between the two parameters $\zeta$ and $\zeta^{\prime}$ is given by ${ }^{1}$

$$
\zeta^{\prime}=\frac{\operatorname{Arth}(|\zeta|)}{|\zeta|} \zeta, \quad \zeta^{\prime *}=\frac{\operatorname{Arth}(|\zeta|)}{|\zeta|} \zeta^{*}, \quad\left|\zeta^{\prime}\right|=\operatorname{Arth}|\zeta|, \quad \frac{\zeta^{\prime}}{\left|\zeta^{\prime}\right|}=\frac{\zeta}{|\zeta|},
$$

${ }^{1}$ Note that the operators $S\left(\zeta^{\prime^{*}}, 0, \zeta^{\prime}\right) \equiv \exp \left(\frac{\zeta^{\prime *}}{2} a^{2}-\frac{\zeta^{\prime}}{2} a^{\dagger 2}\right)$ with complex $\zeta^{\prime}$ do not form a group that means the product of two such operators with different parameters is, in general, not an operator of this type but by a small extension one comes to the $S U(1,1)$ group of squeezing and rotation operators; see Appendix A. 

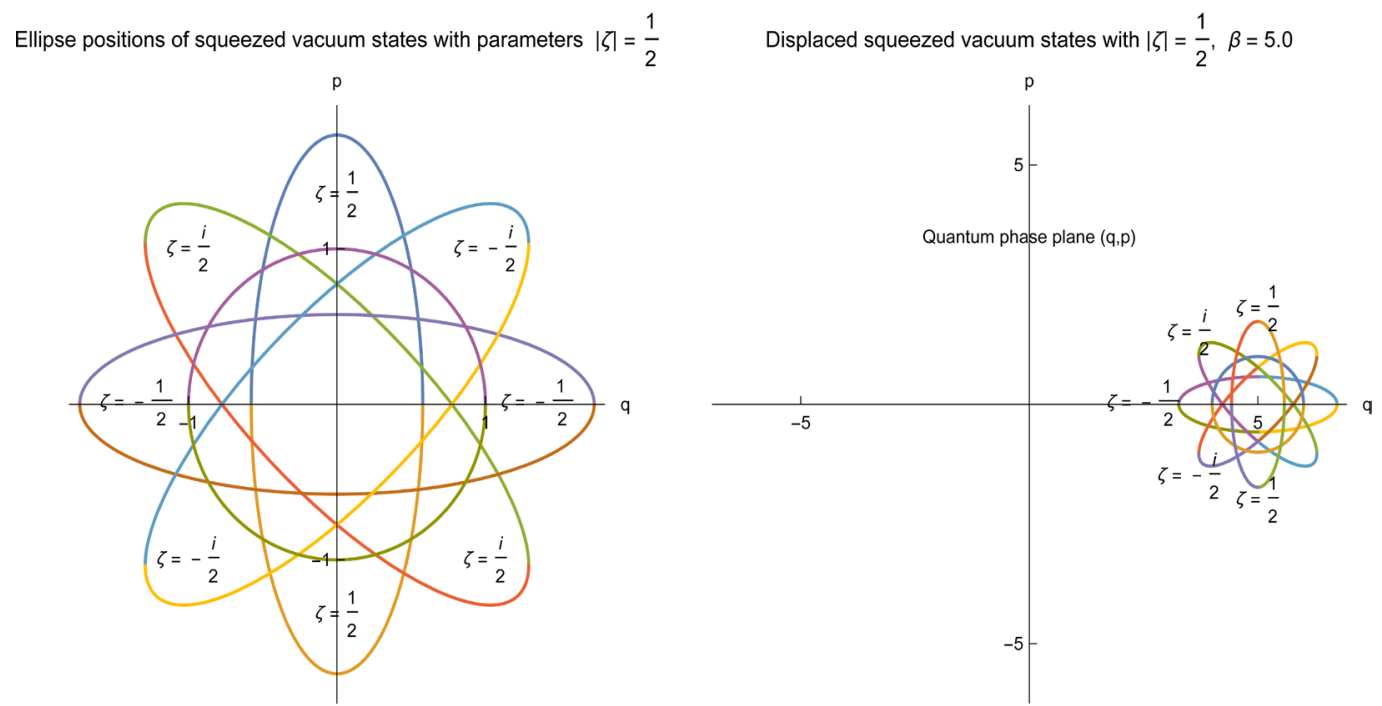

Figure 1. Position of ellipses of squeezed vacuum states in dependence on the phase of squeezing parameter $\zeta$. The drawn squeezing ellipses for $|\zeta|=\frac{1}{2}$ are a contour of equal height of the Wigner quasiprobability $W(q, p)$. The mean value $\bar{N}$ of the number operator $N$ depends for the squeezed vacuum states only on the modulus $|\zeta|$ of the squeezing parameter $\zeta$ and is $\bar{N}=\frac{|\zeta|^{2}}{1-|\zeta|^{2}}$ and the variance is $\overline{(\Delta N)^{2}}=\frac{2|\zeta|^{2}}{\left(1-|\zeta|^{2}\right)^{2}}$ that means $\bar{N}=\frac{1}{3}$ and $\overline{(\Delta N)^{2}}=\frac{8}{9}$ in our case. This shows that all squeezed vacuum states possess a super-Poissonian photon statistics. In the second picture we have shown a displacement of squeezed vacuum states in the quantum phase plane (see from Section 7 on). The squeezing parameter remains the same under displacements. The circle on the left figure corresponds to the vacuum state and on the right figure to a coherent state.

$$
\zeta=\frac{\operatorname{th}\left(\left|\zeta^{\prime}\right|\right)}{\left|\zeta^{\prime}\right|} \zeta^{\prime}, \quad \zeta^{*}=\frac{\operatorname{th}\left(\left|\zeta^{\prime}\right|\right)}{\left|\zeta^{\prime}\right|} \zeta^{\prime *}, \quad|\zeta|=\operatorname{th}\left(\left|\zeta^{\prime}\right|\right)
$$

The parameter $\zeta^{\prime}$ is stretched in comparison to $\zeta$ and takes on the whole complex plane for normalizable states but $\zeta$ and $\zeta^{\prime}$ possess the same directions in the complex plane. It is easy to rewrite the formulae derived in the following from parameters $\left(\zeta, \zeta^{*}\right)$ to parameters $\left(\zeta^{\prime}, \zeta^{\prime *}\right)$ using (5), for example

$$
\frac{1}{1-|\zeta|^{2}}=\operatorname{ch}^{2}\left(\left|\zeta^{\prime}\right|\right), \frac{|\zeta|^{2}}{1-|\zeta|^{2}}=\operatorname{sh}^{2}\left(\left|\zeta^{\prime}\right|\right), \quad \sqrt{\frac{1 \pm|\zeta|}{1 \mp|\zeta|}}=\exp \left( \pm\left|\zeta^{\prime}\right|\right)
$$

The complex parameter $\zeta$ has often some advantages in comparison to the complex parameter $\zeta^{\prime}$ concerning compactness of formulae but sometimes, e.g., in the dynamics to quadratic Hamiltonians in $\left(a, a^{\dagger}\right)$, the representation by $\zeta^{\prime}$ is to prefer and $\zeta$ in literature notations $|\alpha, \zeta\rangle$ corresponds mostly to our $\zeta^{\prime}$. In Appendix A we consider the relations in detail.

The equivalence of the two approaches is given by the following general disentanglement of the unitary squeezing operator in (almost) normal ordering 


$$
\begin{aligned}
& \exp \left\{\frac{\operatorname{Arth}|\zeta|}{|\zeta|}\left(\frac{\zeta^{*}}{2} a^{2}-\frac{\zeta}{2} a^{\dagger 2}\right)\right\} \\
& =\exp \left(-\frac{\zeta}{2} a^{\dagger 2}\right)\left(1-\zeta \zeta^{*}\right)^{\frac{1}{4}\left(a a^{\dagger}+a^{\dagger} a\right)} \exp \left(\frac{\zeta^{*}}{2} a^{2}\right) .
\end{aligned}
$$

The basic relations for squeezing operators between unitary and non-unitary approach were developed already earlier (e.g., [18]) for the $S U(1,1)$ group and we collect the most important relations in Appendix A.

From (2.3) follow the probabilities $p_{n}$ to the photon statistics of squeezed vacuum states

$$
\begin{aligned}
p_{n} & \equiv\langle n \mid 0, \zeta\rangle\langle 0, \zeta \mid n\rangle \\
& =\left\{\begin{array}{l}
p_{2 m}=\sqrt{1-|\zeta|^{2}} \frac{(2 m) !}{2^{2 m} m !^{2}}|\zeta|^{2 m}=\sqrt{1-|\zeta|^{2}} \frac{(2 m-1) ! !}{2^{m} m !}|\zeta|^{2 m} \\
p_{2 m+1}=0 .
\end{array}\right.
\end{aligned}
$$

The sum over the $p_{n}$ are normalized according to

$$
\sum_{n=0}^{\infty} p_{n}=\sqrt{1-|\zeta|^{2}} \sum_{m=0}^{\infty} \frac{(2 m) !}{2^{2 m} m !^{2}}|\zeta|^{2 m}=1,
$$

as one affirms from the Taylor series of the function $\left(1-|\zeta|^{2}\right)^{-\frac{1}{2}}$. Only the probabilities for even $n=2 m$ are non-vanishing and the probabilities $p_{2 m}$ a strictly decreasing $\left(p_{2(m+1)} \leq p_{2 m}\right)$ for increasing $m$.

From the commutation relations

$$
\begin{aligned}
& \exp \left(-\frac{\zeta}{2} a^{\dagger 2}\right) a \exp \left(\frac{\zeta}{2} a^{\dagger 2}\right) \\
& =a-\frac{\zeta}{2}\left[a^{\dagger 2}, a\right]+\frac{1}{2 !}\left(\frac{\zeta}{2}\right)^{2}\left[a^{\dagger 2},\left[a^{\dagger 2}, a\right]\right]-\cdots=a+\zeta a^{\dagger},
\end{aligned}
$$

in connection with $a|0\rangle=0$ follows that the states $|0, \zeta\rangle$ are eigenstates of the operator $a+\zeta a^{\dagger}$ to the eigenvalue zero

$$
0=\exp \left(-\frac{\zeta}{2} a^{\dagger 2}\right) a|0\rangle=\left(a+\zeta a^{\dagger}\right) \exp \left(-\frac{\zeta}{2} a^{\dagger 2}\right)|0\rangle
$$

that means

$$
\left(a+\zeta a^{\dagger}\right)|0, \zeta\rangle=0, \quad \Leftrightarrow \quad a|0, \zeta\rangle=-\zeta a^{\dagger}|0, \zeta\rangle
$$

In representation by canonical operators $(Q, P)$ this is equivalent to

$$
((1+\zeta) Q+\mathrm{i}(1-\zeta) P)|0, \zeta\rangle=0, \quad \Leftrightarrow \quad P|0, \zeta\rangle=\mathrm{i} \frac{1+\zeta}{1-\zeta} Q|0, \zeta\rangle .
$$

Thus both the states $a|0, \zeta\rangle$ and $a^{\dagger}|0, \zeta\rangle$ as well as the states $Q|0, \zeta\rangle$ and $P|0, \zeta\rangle$ are linearly dependent. Furthermore, from (2.12) follows

$$
a^{\dagger k} a|0, \zeta\rangle=-\zeta a^{\dagger k+1}|0, \zeta\rangle, \quad a^{l}|0, \zeta\rangle=-\zeta a^{l-1} a^{\dagger}|0, \zeta\rangle .
$$


If one forms the scalar products of these relations by multiplication with $\langle 0, \zeta|$ one obtains the expectation values of $\overline{a^{\dagger k} a}$ and $-\zeta \overline{a^{\dagger k+1}}$ and the expectation values $\overline{a^{l}}$ and $-\zeta \overline{a^{l-1} a^{\dagger}}$, respectively, with equality relations between them. By differentiations of (2.3) with respect to $\zeta$ and to $\zeta^{*}$ in connection with (2.14) for $k=1$ we find

$$
N|0, \zeta\rangle=-\zeta a^{\dagger 2}|0, \zeta\rangle=2\left(\zeta \frac{\partial}{\partial \zeta}-\zeta^{*} \frac{\partial}{\partial \zeta^{*}}\right)|0, \zeta\rangle
$$

as a further interesting relation which can also be written as eigenvalue equation for $|0, \zeta\rangle$ to eigenvalue zero.

Another interesting characteristics of a state is its (Hilbert-Schmidt) distance to the nearest coherent state which in case of squeezed vacuum states $|0, \zeta\rangle$ is the vacuum state $|0\rangle$. It may be considered as a measure of nonclassicality of a state [20] [21] [22]. For this distance one finds (see (5) for $\zeta$ and $\zeta^{\prime}$ )

$$
\begin{aligned}
d(|0, \zeta\rangle\langle 0, \zeta|,| 0\rangle\langle 0|) & =\sqrt{2(1-\langle 0 \mid 0, \zeta\rangle\langle 0, \zeta \mid 0\rangle)} \\
& =\sqrt{2\left(1-\sqrt{1-|\zeta|^{2}}\right)}=\sqrt{2\left(1-\frac{1}{\operatorname{ch}\left(\left|\zeta^{\prime}\right|\right)}\right)} \\
& =|\zeta|\left(1+\frac{1}{8}|\zeta|^{2}+\cdots\right)=\left|\zeta^{\prime}\right|\left(1-\frac{5}{24}\left|\zeta^{\prime}\right|^{2}+\cdots\right) .
\end{aligned}
$$

It depends only on the modulus of $\zeta$. For strong squeezing $|\zeta| \rightarrow 1$ this distance goes to $\sqrt{2}$ that is the largest distance for normalized states in Hilbert space and means orthogonality of the two states (Figure 2).

\section{Wave Functions of Squeezed Vacuum States and Uncertainty Matrix}

The wave functions of squeezed vacuum states are the scalar products $\langle q \mid 0, \zeta\rangle$ and $\langle p \mid 0, \zeta\rangle$ with the eigenstates $|q\rangle$ and $|p\rangle$ of the operators $Q$ and $P$. Their number representations are

$$
\begin{aligned}
& |q\rangle=\frac{1}{(\hbar \pi)^{\frac{1}{4}}} \exp \left(-\frac{q^{2}}{2 \hbar}\right) \sum_{n=0}^{\infty} \frac{1}{\sqrt{2^{n} n !}} \mathrm{H}_{n}\left(\frac{q}{\sqrt{\hbar}}\right)|n\rangle, \\
& |p\rangle=\frac{1}{(\hbar \pi)^{\frac{1}{4}}} \exp \left(-\frac{p^{2}}{2 \hbar}\right) \sum_{n=0}^{\infty} \frac{\mathrm{i}^{n}}{\sqrt{2^{n} n !}} \mathrm{H}_{n}\left(\frac{p}{\sqrt{\hbar}}\right)|n\rangle,
\end{aligned}
$$

where $\mathrm{H}_{n}(z)$ denotes the Hermite polynomials. They are not normalizable as it is well known and are only normalized by means of the delta function

$$
\begin{aligned}
& \left\langle q \mid q^{\prime}\right\rangle=\delta\left(q-q^{\prime}\right), \quad\left\langle p \mid p^{\prime}\right\rangle=\delta\left(p-p^{\prime}\right), \\
& \langle q \mid p\rangle=\frac{1}{\sqrt{2 \hbar \pi}} \exp \left(\mathrm{i} \frac{q p}{\hbar}\right)=\langle p \mid q\rangle^{*} .
\end{aligned}
$$

Using the number-state representation (3.3) of squeezed vacuum states we find by applying the first of the generating functions (3.3) for even Hermite polynomials 


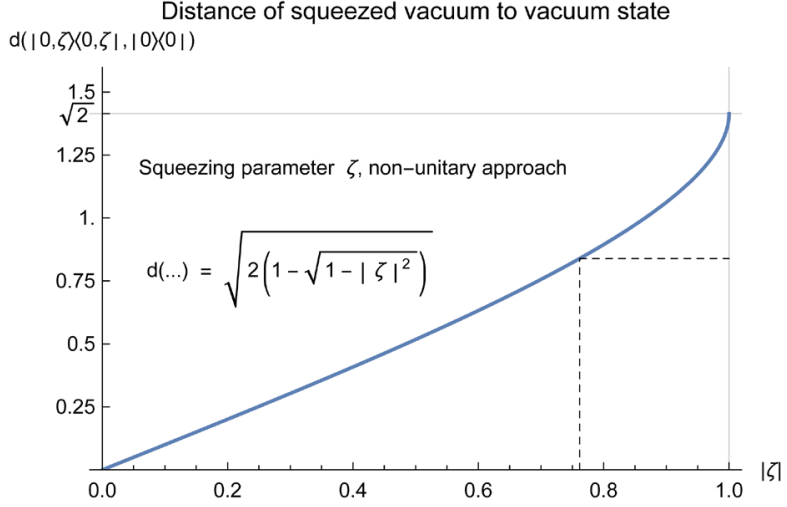

Distance of squeezed vacuum to vacuum state $d(\mid 0, \zeta) \times 0, \zeta|| 0\rangle,\langle 0|)$

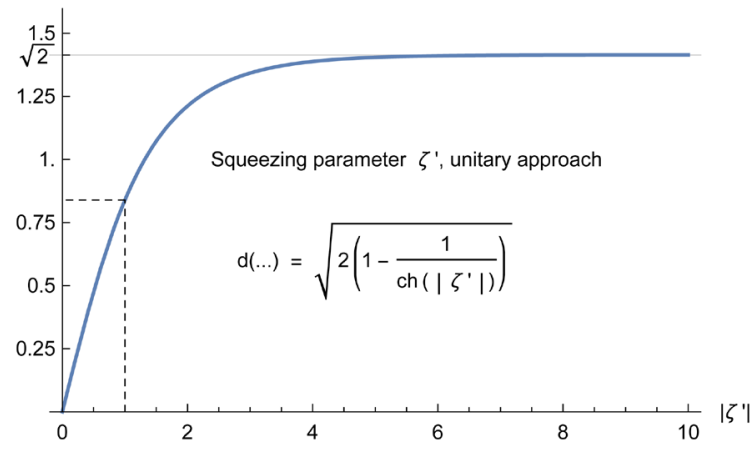

Figure 2. Distance of squeezed vacuum states to vacuum state in dependence on squeezing parameters $\zeta$ and $\zeta^{\prime}$ in non-unitary and in unitary approach. The figures show that for $|\zeta| \rightarrow 1$ the parameter $\left|\zeta^{\prime}\right|$ is stretched up to $\infty$ in comparison to $|\zeta|$. The maximal distance of two pure normalized states in Hilbert space is $\sqrt{2}$.

$$
\begin{aligned}
\langle q \mid 0, \zeta\rangle & =\left(\frac{1-\zeta \zeta^{*}}{\hbar \pi}\right)^{\frac{1}{4}} \exp \left(-\frac{q^{2}}{2 \hbar}\right) \sum_{m=0}^{\infty} \frac{(-1)^{m} \zeta^{m}}{2^{2 m} m !} \mathrm{H}_{2 m}\left(\frac{q}{\sqrt{2 \hbar}}\right) \\
& =\left(\frac{1-\zeta \zeta^{*}}{\hbar \pi}\right)^{\frac{1}{4}} \frac{1}{\sqrt{1-\zeta}} \exp \left(-\frac{(1+\zeta) q^{2}}{(1-\zeta) 2 \hbar}\right) \\
\langle p \mid 0, \zeta\rangle & =\left(\frac{1-\zeta \zeta^{*}}{\hbar \pi}\right)^{\frac{1}{4}} \exp \left(-\frac{p^{2}}{2 \hbar}\right) \sum_{m=0}^{\infty} \frac{\zeta^{m}}{2^{2 m} m !} \mathrm{H}_{2 m}\left(\frac{p}{\sqrt{2 \hbar}}\right) \\
& =\left(\frac{1-\zeta \zeta^{*}}{\hbar \pi}\right)^{\frac{1}{4}} \frac{1}{\sqrt{1+\zeta}} \exp \left(-\frac{(1-\zeta) p^{2}}{(1+\zeta) 2 \hbar}\right)
\end{aligned}
$$

From this follows

$$
\begin{gathered}
W(q) \equiv\langle q \mid 0, \zeta\rangle\langle 0, \zeta \mid q\rangle=\sqrt{\frac{1-\zeta \zeta^{*}}{(1-\zeta)\left(1-\zeta^{*}\right) \hbar \pi}} \exp \left(-\frac{\left(1-\zeta \zeta^{*}\right) q^{2}}{(1-\zeta)\left(1-\zeta^{*}\right) \hbar}\right), \\
W(p) \equiv\langle p \mid 0, \zeta\rangle\langle 0, \zeta \mid p\rangle=\sqrt{\frac{1-\zeta \zeta^{*}}{(1+\zeta)\left(1+\zeta^{*}\right) \hbar \pi}} \exp \left(-\frac{\left(1-\zeta \zeta^{*}\right) p^{2}}{(1+\zeta)\left(1+\zeta^{*}\right) \hbar}\right),
\end{gathered}
$$


with the normalization

$$
\int_{-\infty}^{+\infty} \mathrm{d} q W(q)=\int_{-\infty}^{+\infty} \mathrm{d} p W(p)=1 .
$$

The functions $W(q)$ and $W(p)$ are the Wigner quasiprobability $W(q, p)$ integrated over one of the canonical variable $p$ or $q$. The functions (3.4) remain invariant by interchanging the squeezing parameter $\zeta$ with its complex conjugate $\zeta \leftrightarrow \zeta^{*}$. This shows in an example that, in general, a state (here $|0, \zeta\rangle)$ cannot uniquely be reconstructed from $W(q)$ and $W(p)$ alone.

The functions $W(q)$ and $W(p)$ are two normalized Gaussian distribution with the expectation values

$$
\bar{Q}=0, \quad \bar{P}=0, \quad \overline{(\Delta Q)^{2}}=\frac{(1-\zeta)\left(1-\zeta^{*}\right)}{1-\zeta \zeta^{*}} \frac{\hbar}{2}, \quad \overline{(\Delta P)^{2}}=\frac{(1+\zeta)\left(1+\zeta^{*}\right)}{1-\zeta \zeta^{*}} \frac{\hbar}{2}
$$

The product of their uncertainties $\overline{(\Delta Q)^{2}}$ and $\overline{(\Delta P)^{2}}$ (note inequality $z^{2}+z^{* 2} \leq 2 z z^{*}$ for arbitrary complex $z=x+i y$ in contrast to $x^{2}+y^{2} \geq 2 x y$ for arbitrary real $x$ and $y$ )

$$
\overline{(\Delta Q)^{2}} \frac{(\Delta P)^{2}}{\left(1-\zeta \zeta^{*}\right)^{2}} \frac{\left(1-\zeta^{2}\right)\left(1-\zeta^{* 2}\right)}{4} \geq \frac{\hbar^{2}}{4}
$$

It depends on the phase $\chi$ of the complex squeezing parameter $\zeta=|\zeta| \mathrm{e}^{\mathrm{i} \chi}$ that means on the position of the principal axes of squeezing in comparison to the axes of the canonical coordinates $(q, p)$ (see Figure 1). In contrast, the sum of the uncertainties

$$
\overline{(\Delta Q)^{2}}+\overline{(\Delta P)^{2}}=\frac{1+\zeta \zeta^{*}}{1-\zeta \zeta^{*}} \hbar
$$

does not depend on the phase of the squeezing parameter $\zeta$. For real squeezing parameter $\zeta=\zeta^{*}$ we find for the uncertainties

$$
\overline{(\Delta Q)^{2}}=\frac{1-\zeta}{1+\zeta} \frac{\hbar}{2}, \quad \overline{(\Delta P)^{2}}=\frac{1+\zeta}{1-\zeta} \frac{\hbar}{2}, \quad\left(\zeta=\zeta^{*}\right)
$$

and their product is equal to $\frac{\hbar^{2}}{4}$ the minimal possible one. The principal axes of the squeezing ellipses are then in direction of the coordinate axes $(q, p)$ (see Figure 1). Clearly, it is not satisfying to consider squeezed vacuum states $|0, \zeta\rangle$ with real $\zeta$ as minimum uncertainty states and such with complex $\zeta$ which are only rotated in the phase plane (Figure 1), in general, not as minimum uncertainty states. The satisfying solution of this problem is to consider in addition the uncertainty correlation $\frac{1}{2} \overline{\Delta Q \Delta P+\Delta P \Delta Q}$ [23] [24].

The uncertainty correlation $\frac{1}{2} \overline{Q P+P Q}$ arises in a natural way as nondiagonal elements of the (symmetrical) uncertainty matrix $\mathrm{S}$ if we consider the cumulant expansion of the Wigner quasiprobability $W(q, p)$ or the corresponding expansion of its Fourier transform $\tilde{W}(u, v)$ [24] 


$$
\begin{aligned}
\mathrm{S} & \equiv\left(\begin{array}{cc}
\overline{(\Delta Q)^{2}} & \frac{1}{2} \overline{\Delta Q \Delta P+\Delta P \Delta Q} \\
\frac{1}{2} \overline{\Delta Q \Delta P+\Delta P \Delta Q} & \overline{(\Delta P)^{2}}
\end{array}\right) \\
& =\left(\begin{array}{cc}
\overline{\Delta a \Delta a^{\dagger}+\Delta a^{\dagger} \Delta a}+\left(\overline{(\Delta a)^{2}}+\overline{\left(\Delta a^{\dagger}\right)^{2}}\right) & -\mathrm{i}\left(\overline{(\Delta a)^{2}}-\overline{\left(\Delta a^{\dagger}\right)^{2}}\right) \\
-\mathrm{i}\left(\overline{(\Delta a)^{2}}-\overline{\left(\Delta a^{\dagger}\right)^{2}}\right) & \overline{\Delta a \Delta a^{\dagger}+\Delta a^{\dagger} \Delta a}-\left(\overline{(\Delta a)^{2}}+\overline{\left(\Delta a^{\dagger}\right)^{2}}\right)
\end{array}\right)
\end{aligned}
$$

It is also called variance matrix [23] and is related to the covariance matrix [10] (3.p. 61). The trace of the uncertainty matrix $s$ denoted by $\langle S\rangle$

$$
\langle\mathrm{S}\rangle=\overline{(\Delta Q)^{2}}+\overline{(\Delta P)^{2}}=\left(\overline{\Delta a \Delta a^{\dagger}+\Delta a^{\dagger} \Delta a}\right) \hbar,
$$

is the uncertainty sum and the determinant of the matrix $S$ denoted by [S] is essentially the uncertainty product but modified by the uncertainty correlations

$$
\begin{aligned}
{[\mathrm{S}] } & =\overline{(\Delta Q)^{2}} \overline{(\Delta P)^{2}}-\frac{1}{4}(\overline{\Delta Q \Delta P+\Delta P \Delta Q})^{2} \\
& =\left\{\left(\overline{\Delta a \Delta a^{\dagger}+\Delta a^{\dagger} \Delta a}\right)^{2}-4 \overline{(\Delta a)^{2}} \overline{\left(\Delta a^{\dagger}\right)^{2}}\right\} \frac{\hbar^{2}}{4} \geq \frac{\hbar^{2}}{4} .
\end{aligned}
$$

The chain of inequalities which generalizes the basic uncertainty relation of quantum mechanics is ([18] [24])

$$
\frac{\hbar}{2} \leq \sqrt{[\mathrm{S}]} \leq \sqrt{\overline{(\Delta Q)^{2}} \overline{(\Delta P)^{2}}} \leq\langle\mathrm{S}\rangle .
$$

Both quantities $\langle S\rangle$ and $[S]$ are invariant with respect to rotations and displacement of the states in the quantum phase plane and $\langle S\rangle$ is additionally invariant with respect to squeezing [24].

For squeezed vacuum states $|0, \zeta\rangle$ we find using their number representation (2.2)

$$
\frac{1}{2}(\overline{\Delta Q \Delta P+\Delta P \Delta Q})=-\mathrm{i}\left(\overline{(\Delta a)^{2}}-\overline{\left(\Delta a^{\dagger}\right)^{2}}\right) \frac{\hbar}{2}=\mathrm{i} \frac{\zeta-\zeta^{*}}{1-\zeta \zeta^{*}} \frac{\hbar}{2},
$$

and therefore for the modified uncertainty product [S] in (3.12) using the explicit expressions (3.7) and (3.14)

$$
[\mathrm{S}]=\frac{\hbar^{2}}{4}
$$

This modified uncertainty product for squeezed vacuum states does no more depend on the position of the principal axes of squeezing ellipses in phase plane of canonical coordinates $(q, p)$ shown in Figure 1 . It is the minimum possible one characterizing the states as minimum uncertainty states in this generalized sense.

By a rotation of the canonical coordinates $(q, p)$ to new canonical coordinates $\left(q^{\prime}, p^{\prime}\right)$ one may bring them in the position of the principal axes of the squeezing ellipses and since this is fully obvious we do not give the explicit 
transformation relations. However, this suggests that it is better not to exclude squeezed vacuum states with arbitrary positions of the squeezing ellipses from the minimum uncertainty states.

\section{Bargmann Representation and Quasiprobabilities for Squeezed Vacuum States}

The Bargmann representation of a state is a representation by an analytic function which, in particular, leads immediately to the Husimi-Kano quasiprobability $Q\left(\alpha, \alpha^{*}\right)$ [25] (chap. 7) and [26]. For this purpose we calculate the scalar product of a state with the analytic (but non-normalized) coherent states $\| \alpha\rangle \equiv \exp \left(\frac{\alpha \alpha^{*}}{2}\right)|\alpha\rangle=\sum_{n=0}^{\infty} \frac{\alpha^{n}}{\sqrt{n !}}|n\rangle$ with arbitrary complex $\alpha$. For the squeezed vacuum state $|0, \zeta\rangle$ this provides its Bargmann representations

$$
\begin{aligned}
\exp \left(\frac{\alpha \alpha^{*}}{2}\right)\langle 0, \zeta \mid \alpha\rangle & =\left(1-\zeta \zeta^{*}\right)^{\frac{1}{4}} \sum_{m=0}^{\infty} \frac{(-1)^{m}}{2^{m} m !} \zeta^{* m} \alpha^{2 m} \\
& =\left(1-\zeta \zeta^{*}\right)^{\frac{1}{4}} \exp \left(-\frac{\zeta^{*}}{2} \alpha^{2}\right)=\left(\exp \left(\frac{\alpha \alpha^{*}}{2}\right)\langle\alpha \mid 0, \zeta\rangle\right)^{*}
\end{aligned}
$$

From this one obtains the Husimi-Kano quasiprobability $Q\left(\alpha, \alpha^{*}\right)$ for density operator $\rho=|0, \zeta\rangle\langle 0, \zeta|$

$$
\begin{aligned}
& Q\left(\alpha, \alpha^{*}\right) \equiv \frac{\langle\alpha \mid 0, \zeta\rangle\langle 0, \zeta \mid \alpha\rangle}{\pi}=\frac{\sqrt{1-\zeta \zeta^{*}}}{\pi} \exp \left\{-\left(\alpha \alpha^{*}+\frac{\zeta^{*}}{2} \alpha^{2}+\frac{\zeta}{2} \alpha^{* 2}\right)\right\}, \\
& \int \frac{\mathrm{i}}{2} \mathrm{~d} \alpha \wedge \mathrm{d} \alpha^{*} Q\left(\alpha, \alpha^{*}\right)=1 .
\end{aligned}
$$

In representation by real canonical variables $(q, p)$ this is

$$
\begin{aligned}
& Q(q, p)=\frac{\sqrt{1-\zeta \zeta^{*}}}{2 \hbar \pi} \exp \left\{-\frac{2}{\hbar}\left(\left(1+\frac{\zeta+\zeta^{*}}{2}\right) q^{2}+\left(1-\frac{\zeta+\zeta^{*}}{2}\right) p^{2}-\mathrm{i}\left(\zeta-\zeta^{*}\right)\right) q p\right\}, \\
& \int \mathrm{d} q \wedge \mathrm{d} p Q(q, p)=1 .
\end{aligned}
$$

There are different possibilities to calculate the Wigner quasprobability [27] [28]. In most convenient way the Wigner quasiprobability $W\left(\alpha, \alpha^{*}\right)$ of an arbitrary squeezed state can be calculated from the Wigner quasiprobability $W_{0}\left(\alpha, \alpha^{*}\right)$ of the corresponding non-squeezed state using the relation (4.9) of Appendix B. The parameters for our non-unitary approach (4.4) are derived in (4.18) where we have to set $\xi=\zeta^{*}$ and have

$$
W\left(\alpha, \alpha^{*}\right)=W_{0}\left(\frac{\alpha+\zeta \alpha^{*}}{\sqrt{1-\zeta \zeta^{*}}}, \frac{\alpha^{*}+\zeta^{*} \alpha}{\sqrt{1-\zeta \zeta^{*}}}\right) .
$$

Taking into account the well-known Wigner quasiprobability for the vacuum state 


$$
W_{0}\left(\alpha, \alpha^{*}\right)=\frac{2}{\pi} \exp \left(-2 \alpha \alpha^{*}\right), \quad(\rho=|0\rangle\langle 0|),
$$

we obtain

$$
\begin{aligned}
& W\left(\alpha, \alpha^{*}\right)=\frac{2}{\pi} \exp \left\{-2 \frac{\left(\alpha+\zeta \alpha^{*}\right)\left(\alpha^{*}+\zeta^{*} \alpha\right)}{1-\zeta \zeta^{*}}\right\} \\
& =\frac{2}{\pi} \exp \left\{-2 \frac{\left(1+\zeta \zeta^{*}\right) \alpha \alpha^{*}+\zeta^{*} \alpha^{2}+\zeta \alpha^{* 2}}{1-\zeta \zeta^{*}}\right\}, \quad \int \frac{\mathrm{i}}{2} \mathrm{~d} \alpha \wedge \mathrm{d} \alpha^{*} W\left(\alpha, \alpha^{*}\right)=1 .
\end{aligned}
$$

In representation by the real canonical variables $(q, p)$

$$
\begin{aligned}
& W(q, p) \\
& =\frac{1}{\hbar \pi} \exp \left\{-\frac{(1+\zeta)\left(1+\zeta^{*}\right) q^{2}+(1-\zeta)\left(1-\zeta^{*}\right) p^{2}-\mathrm{i}\left(\zeta-\zeta^{*}\right) 2 q p}{\left(1-\zeta \zeta^{*}\right) \hbar}\right\} \\
& =\frac{1}{\hbar \pi} \exp \left\{-\frac{((1+\zeta) q+\mathrm{i}(1-\zeta) p)\left(\left(1+\zeta^{*}\right) q-\mathrm{i}\left(1-\zeta^{*}\right) p\right)}{\left(1-\zeta \zeta^{*}\right) \hbar}\right\}, \\
& \int \mathrm{d} q \wedge \mathrm{d} p W(q, p)=1 .
\end{aligned}
$$

Another often easy way to calculate the Wigner quasiprobability $W\left(\alpha, \alpha^{*}\right)$ is to make first the normal ordering of the operator involved in the representation (4.1) that leads to [22]

$$
\begin{aligned}
W\left(\alpha, \alpha^{*}\right) & =\left\langle\rho \exp \left(-a \frac{\partial}{\partial \alpha}-a^{\dagger} \frac{\partial}{\partial \alpha^{*}}\right)\right\rangle \delta\left(\alpha, \alpha^{*}\right) \\
& =\left\langle\rho \exp \left(-a^{\dagger} \frac{\partial}{\partial \alpha^{*}}\right) \exp \left(-a \frac{\partial}{\partial \alpha}\right)\right\rangle \exp \left(\frac{1}{2} \frac{\partial^{2}}{\partial \alpha \partial \alpha^{*}}\right) \delta\left(\alpha, \alpha^{*}\right) \\
& =\left\langle\rho \exp \left(-a^{\dagger} \frac{\partial}{\partial \alpha^{*}}\right) \exp \left(-a \frac{\partial}{\partial \alpha}\right)\right\rangle \frac{2}{\pi} \exp \left(-2 \alpha \alpha^{*}\right)
\end{aligned}
$$

For example, one obtains then immediately from it the already used Wigner quasiprobability for the vacuum state (4.5) since the application of the operator $\exp \left(-a \frac{\partial}{\partial \alpha}\right)$ to the state $|0\rangle$ and of $\exp \left(-a^{\dagger} \frac{\partial}{\partial \alpha^{*}}\right)$ from the right to $\langle 0|$ reproduces them. In the real representation one may use the following equivalent definitions where the first corresponds to the definition given by Wigner [27] [28] for pure states (written here with density operator $\rho$ and with Dirac's notations for states)

$$
\begin{aligned}
W(q, p) & =\frac{1}{\hbar \pi} \int_{-\infty}^{+\infty} \mathrm{d} x\langle q-x|\rho| q+x\rangle \exp \left(\mathrm{i} \frac{2 p x}{\hbar}\right) \\
& =\frac{1}{\hbar \pi} \int_{-\infty}^{+\infty} \mathrm{d} y\langle p+y|\rho| p-y\rangle \exp \left(\mathrm{i} \frac{2 q y}{\hbar}\right) .
\end{aligned}
$$

We checked (4.7) by these formulae using the wave functions $\langle q \mid 0, \zeta\rangle$ and $\langle p \mid 0, \zeta\rangle$ derived in (3.3).

Without presenting its detailed calculation let us give the more general 
quasiprobabilities $F_{r}\left(\alpha, \alpha^{*}\right)$ with the parameter $r$ and defined by [29] (*) means convolution)

$$
\begin{aligned}
F_{r}\left(\alpha, \alpha^{*}\right) & =\exp \left(\frac{r}{2} \frac{\partial^{2}}{\partial \alpha \partial \alpha^{*}}\right) W\left(\alpha, \alpha^{*}\right) \\
& =\frac{2}{\pi \sqrt{r^{2}}} \exp \left(-\frac{2 \alpha \alpha^{*}}{r}\right) * W\left(\alpha, \alpha^{*}\right),
\end{aligned}
$$

Our result for the squeezed vacuum states $|0, \zeta\rangle$ is

$$
\begin{aligned}
F_{r}\left(\alpha, \alpha^{*}\right)= & \frac{1}{\pi} \sqrt{\left(\frac{1+r}{2}\right)^{2}-\left(\frac{1-r}{2}\right)^{2} \zeta \zeta^{*}} \\
& \times \exp \left\{-\frac{\left(\frac{1+r}{2}+\frac{1-r}{2} \zeta \zeta^{*}\right) \alpha \alpha^{*}+\frac{\zeta^{*}}{2} \alpha^{2}+\frac{\zeta}{2} \alpha^{* 2}}{\left(\frac{1+r}{2}\right)^{2}-\left(\frac{1-r}{2}\right)^{2} \zeta \zeta^{*}}\right\} .
\end{aligned}
$$

For $r=0$ one obtains the Wigner quasiprobability $W\left(\alpha, \alpha^{*}\right)$, for $r=+1$ the Husimi-Kano quasiprobability $Q\left(\alpha, \alpha^{*}\right)$ and for $r=-1$ the GlauberSudarshan quasiprobability $P\left(\alpha, \alpha^{*}\right)$. The Glauber-Sudarshan quasiprobability $P\left(\alpha, \alpha^{*}\right)$ for squeezed vacuum states is a singular generalized function and makes for $\zeta=0$ the transition to the delta function $\delta\left(\alpha, \alpha^{*}\right)$. The representation of (4.10) by real canonical variables $(q, p)$ is easily to make. Furthermore, with some calculation one may bring all quasiprobabilities in representation by real variable to principal axes form that, however, we do not demonstrate here.

\section{Expectation Values of Powers of Number Operator and Related Ordered Operators for Squeezed Vacuum States}

We now calculate expectation values for the squeezed vacuum states. In particular interesting are the expectation values of ordered powers of the annihilation and creation operators $\left(a, a^{\dagger}\right)$. We begin with the special expectation values $\overline{a^{\dagger k} a^{k}}$ since from these expectation values one may calculate the expectation values $\overline{N^{l}}$ of the number operator $N$.

Using the number representation (2.3) of the squeezed vacuum states $|0, \zeta\rangle$ we proceed quickly for the expectation values $\overline{a^{\dagger k} a^{k}}$ which depend only on $|\zeta| \equiv \sqrt{\zeta \zeta^{*}}$ to the following intermediate result

$$
\begin{aligned}
\overline{a^{\dagger k} a^{k}} & \equiv\left\langle 0, \zeta\left|a^{\dagger k} a^{k}\right| 0, \zeta\right\rangle=\sqrt{1-|\zeta|^{2}} \sum_{m=0}^{\infty} \frac{(2 m) !^{2}}{2^{2 m} m !^{2}(2 m-k) !}|\zeta|^{2 m} \\
& =\sqrt{1-|\zeta|^{2}}|\zeta|^{k} \frac{\partial^{k}}{\partial|\zeta|^{k}} \sum_{m=0}^{\infty} \frac{(2 m) !}{2^{2 m} m !^{2}}|\zeta|^{2 m} \\
& =\sqrt{1-|\zeta|^{2}}|\zeta|^{k} \frac{\partial^{k}}{\partial|\zeta|^{k}} \frac{1}{\sqrt{1-|\zeta|^{2}}},
\end{aligned}
$$


where we used that the last sum on the right-hand side is the Taylor series of $\left(1-|\zeta|^{2}\right)^{-\frac{1}{2}}$. This result can be expressed by the Ultraspherical polynomials $\mathrm{P}_{n}^{(\alpha, \alpha)}(u)$ as special case $\alpha=\beta$ of the Jacobi polynomials $\mathrm{P}_{n}^{(\alpha, \beta)}(u)$ (e.g., [30] [31]) in two essentially different ways. We represent this in a slightly more general form as needed here in Appendix C. The result specialized for (5.1) is

$$
\begin{aligned}
\overline{a^{\dagger k} a^{k}} & =k !\left(-\frac{2|\zeta|}{1-|\zeta|^{2}}\right)^{k} \mathrm{P}_{k}^{\left(-\frac{1}{2}-k,-\frac{1}{2}-k\right)}(|\zeta|) \\
& =k !\left(-\frac{\mathrm{i}|\zeta|}{\sqrt{1-|\zeta|^{2}}}\right)^{k} \mathrm{P}_{k}\left(\frac{\mathrm{i}|\zeta|}{\sqrt{1-|\zeta|^{2}}}\right),
\end{aligned}
$$

where $\mathrm{P}_{n}(u) \equiv \mathrm{P}_{n}^{(0,0)}(u)$ are the Legendre polynomials as special case of the Jacobi polynomials $\mathrm{P}_{n}^{(\alpha, \beta)}(u)$. More explicitly this provides

$$
\overline{a^{\dagger k} a^{k}}=k !\left(\frac{|\zeta|}{2\left(1-|\zeta|^{2}\right)}\right)^{k}\left[\sum_{j=0}^{\left.\frac{k}{2}\right]} \frac{k !}{j !^{2}(k-2 j) !}(2|\zeta|)^{k-2 j} \geq 0 .\right.
$$

Remarkable in these transformations is that we could split an essential factor $\left(\frac{|\zeta|}{1-|\zeta|^{2}}\right)^{k}$ multiplied by a polynomial in comparison to the infinite sums in (5.1).

The expectation values of symmetrically ordered power operators $\overline{\mathcal{S}\left\{a^{\dagger k} a^{k}\right\}}$ are connected with $\overline{a^{\dagger k} a^{k}}$ by (see, e.g., Equation (7.6) in [22])

$$
\overline{\mathcal{S}\left\{a^{\dagger k} a^{k}\right\}}=\sum_{l=0}^{k} \frac{k !^{2}}{l !(k-l) !^{2}}\left(\frac{1}{2}\right)^{l} \overline{a^{\dagger k-l} a^{k-l}} .
$$

Inserting for $\overline{a^{\dagger k-l} a^{k-l}}$ the result (5.3) one may transform the arising double sum by reordering to

$$
\begin{aligned}
\overline{\mathcal{S}\left\{a^{+k} a^{k}\right\}} & =\frac{k !}{\left(2\left(1-|\zeta|^{2}\right)\right)^{k}} \sum_{j=0}^{\left[\frac{k}{2}\right]} \frac{k !}{j !^{2}(k-2 j) !}|\zeta|^{2 j}\left(1+|\zeta|^{2}\right)^{k-2 j} \\
& =\frac{k !}{\left(2\left(1-|\zeta|^{2}\right)\right)^{k}} \sum_{l=0}^{k}\left(\frac{k !}{l !(k-l) !}\right)^{2}|\zeta|^{2 l} .
\end{aligned}
$$

The transition from the first line to the second line is possible after Taylor series expansion of $\left(1+|\zeta|^{2}\right)^{k-2 j}$ and applying then Vandermonde's convolution identity which provides a particularly interesting representation with the squared binomial coefficients involved. One may also directly make the transition to representations of the result by the Jacobi polynomials and by its special case of Legendre polynomials using their explicit representations and transformation formulae as follows 


$$
\begin{aligned}
\overline{\mathcal{S}\left\{a^{\dagger k} a^{k}\right\}} & =\frac{k !}{2^{k}} \mathrm{P}_{k}\left(\frac{1+|\zeta|^{2}}{1-|\zeta|^{2}}\right)=\frac{k !}{\left(2\left(1-|\zeta|^{2}\right)\right)^{k}} \mathrm{P}_{k}^{(0,-2 k-1)}\left(1-2|\zeta|^{2}\right) \\
& =k !\left(\frac{|\zeta|^{2}}{2\left(1-|\zeta|^{2}\right)}\right)^{k} \mathrm{P}_{k}^{(0,-2 k-1)}\left(1-\frac{2}{|\zeta|^{2}}\right) .
\end{aligned}
$$

In Appendix E we give some first members of the explicit representation of the sequence $\overline{\mathcal{S}\left\{a^{\dagger k} a^{k}\right\}}$.

The expectation values $\overline{N^{k}}$ of powers of the number operator can be calculated from the expectation values $\overline{a^{\dagger k} a^{k}}$ by the relation

$$
\overline{N^{k}}=\sum_{l=0}^{k} \mathrm{~S}(k, l) \overline{a^{\dagger l} a^{l}},
$$

where $\mathrm{S}(k, l)$ denotes the Stirling numbers of second kind. We could not find up to now a closed representation of the coefficients in front of powers of $|\zeta|^{2}$ in the polynomial in the numerator over the denominator $\left(1-|\zeta|^{2}\right)^{k}$ in $\overline{N^{k}}$ and we give explicit results in Appendix E up to $k=5$.

From the calculated expectation values we find in first order

$$
\overline{a^{\dagger} a}=\bar{N}=\frac{|\zeta|^{2}}{1-|\zeta|^{2}}, \quad \overline{\mathcal{S}\left\{a^{\dagger} a\right\}}=\frac{1+|\zeta|^{2}}{2\left(1-|\zeta|^{2}\right)}=\bar{N}+\frac{1}{2},
$$

and in second order (see also Appendix $\mathrm{E}$ for more and higher-order expectation values)

$$
\begin{gathered}
\overline{a^{\dagger 2} a^{2}}=\frac{|\zeta|^{2}\left(1+2|\zeta|^{2}\right)}{\left(1-|\zeta|^{2}\right)^{2}}=\bar{N}(3 \bar{N}+1), \quad \overline{N^{2}}=\frac{|\zeta|^{2}\left(2+|\zeta|^{2}\right)}{\left(1-|\zeta|^{2}\right)^{2}}=\bar{N}(3 \bar{N}+2), \\
\overline{\mathcal{S}\left\{a^{\dagger 2} a^{2}\right\}}=\frac{1+4|\zeta|^{2}+|\zeta|^{4}}{2\left(1-|\zeta|^{2}\right)^{2}}=3 \bar{N}(\bar{N}+1)+\frac{1}{2} .
\end{gathered}
$$

From this follows for squeezed vacuum states using $|\zeta|^{2}=\frac{\bar{N}}{\bar{N}+1}<1$

$$
\overline{(\Delta N)^{2}} \equiv \overline{N^{2}}-(\bar{N})^{2}=\frac{2|\zeta|^{2}}{\left(1-|\zeta|^{2}\right)^{2}}=2 \bar{N}(\bar{N}+1),
$$

that means the number uncertainty for squeezed vacuum states is larger than, for example, for coherent states $\left(\overline{(\Delta N)^{2}}=\bar{N}\right)$ and furthermore

$$
\begin{gathered}
\overline{a^{\dagger 2} a^{2}}-\left(\overline{a^{\dagger} a}\right)^{2}=\frac{|\zeta|^{2}\left(1+|\zeta|^{2}\right)}{\left(1-|\zeta|^{2}\right)^{2}}=\overline{(\Delta N)^{2}}-\bar{N}=\bar{N}(2 \bar{N}+1), \\
\overline{\mathcal{S}\left\{a^{\dagger 2} a^{2}\right\}}-\left(\overline{\mathcal{S}\left\{a^{\dagger} a\right\}}\right)^{2}=\frac{1+6|\zeta|^{2}+|\zeta|^{4}}{4\left(1-|\zeta|^{2}\right)^{2}}=\frac{1}{4}+2 \bar{N}(\bar{N}+1) \geq \frac{1}{4} .
\end{gathered}
$$


This shows that sub-Poissonian statistics $\overline{a^{\dagger 2} a^{2}}-\left(\overline{a^{\dagger} a}\right)^{2}<0$ does not exist for squeezed vacuum states and that $\overline{\mathcal{S}\left\{a^{\dagger 2} a^{2}\right\}}-\left(\overline{\mathcal{S}\left\{a^{\dagger} a\right\}}\right)^{2} \geq \frac{1}{4}$ satisfies the general inequality for this quantity [22].

\section{Further Expectation Values for Squeezed Vacuum States}

In this Section we calculate more general expectation values for the squeezed vacuum states of the form $\overline{a^{\dagger k} a^{l}}={\overline{a^{\dagger l} a^{k}}}^{*}$ than in the preceding Section. They are only non-vanishing if the difference $k-l$ is an even number $2 m$. This follows from symmetry considerations of the squeezed vacuum states or from their number-state representation which contains only even number states $|2 m\rangle$ Therefore, we now calculate separately the expectation values $\overline{a^{\dagger 2 k} a^{2 l}}$ and $\overline{a^{\dagger 2 k+1} a^{2 l+1}}$. They depend on $\zeta$ and $\zeta^{*}$ separately and, therefore, we use here the pair of variables $\left(\zeta, \zeta^{*}\right)$ for the representation of the results.

For $\overline{a^{\dagger 2 k} a^{2 l}}$ using (2.2) and doubling relations for the Gamma function applied to $(2 x)$ ! we arrive at the intermediate result

$$
\begin{aligned}
& \overline{a^{\dagger 2 k} a^{2 l}} \equiv\left\langle 0, \zeta\left|a^{\dagger 2 k} a^{2 l}\right| 0, \zeta\right\rangle \\
& =\sqrt{\frac{1-\zeta \zeta^{*}}{\pi}}(-2)^{k+l} \zeta^{* k} \zeta^{l} \sum_{j=0}^{\infty} \frac{\left(j+k-\frac{1}{2}\right) !\left(j+l-\frac{1}{2}\right) !}{j !\left(j-\frac{1}{2}\right) !}\left(\zeta \zeta^{*}\right)^{j}, \\
& \left(\left(-\frac{1}{2}\right) ! \equiv \sqrt{\pi}\right) .
\end{aligned}
$$

By comparison of this expression with explicit expressions for the Hypergeometric function ${ }_{2} \mathrm{~F}_{1}(\alpha, \beta ; \gamma ; z)$ we see that this is a polynomial case with the Jacobi polynomials $\mathrm{P}_{n}^{(\alpha, \beta)}(u)$ involved. The main polynomial case of the Hypergeometric function is

$$
\begin{aligned}
{ }_{2} \mathrm{~F}_{1}(-n, \beta ; \gamma ; z) & =\frac{k !(\gamma-1) !}{(n+\gamma-1) !} \mathrm{P}_{n}^{(\gamma-1, \beta-\gamma-n)}(1-2 z) \\
& =\frac{n !(\gamma-1) !}{(n+\gamma-1) !} z^{n} \mathrm{P}_{n}^{(-\beta-n, \beta-\gamma-n)}\left(1-\frac{2}{z}\right) \\
& ={ }_{2} \mathrm{~F}_{1}(\beta,-n ; \gamma ; z),
\end{aligned}
$$

and the other possible polynomial case is ( see [32] Section 5 there)

$$
\mathrm{z}^{n}{ }_{2} \mathrm{~F}_{1}\left(-\frac{n}{2},-\frac{n-1}{2} ; \alpha+1 ;-\frac{1}{\mathrm{z}^{2}}\right)=\frac{n ! \alpha !}{(n+\alpha) !}\left(\sqrt{1+\mathrm{z}^{2}}\right)^{n} \mathrm{P}_{n}^{(\alpha, \alpha)}\left(\frac{\mathrm{z}}{\sqrt{1+z^{2}}}\right)
$$

By transformation relations of the Hypergeometric function, in general, and in possible special cases we find the relation between the Jacobi polynomials

$$
(1-z)^{n} \mathrm{P}_{n}^{(\alpha, \beta)}\left(\frac{1+z}{1-z}\right)=\mathrm{P}_{n}^{(\alpha,-2 n-\alpha-\beta-1)}(1-2 z),
$$

and in special case if one of the upper indices is an integer 


$$
\frac{n !}{(n+\beta) !}\left(\frac{2}{u-1}\right)^{n} \mathrm{P}_{n}^{(m-n, \beta)}(u)=\frac{m !}{(m+\beta) !}\left(\frac{2}{u-1}\right)^{m} \mathrm{P}_{m}^{(n-m, \beta)}(u) .
$$

Together with the more trivial relation

$$
\mathrm{P}_{n}^{(\alpha, \beta)}(u)=(-1)^{n} \mathrm{P}_{n}^{(\beta, \alpha)}(-u),
$$

this provides many possibilities to represent our final results.

Our final result admits the following two equivalent representations showing some symmetry with respect to interchanging $k \leftrightarrow l$

$$
\begin{aligned}
\overline{a^{\dagger 2 k} a^{2 l}} & =(-2)^{k+l} \frac{k !\left(l-\frac{1}{2}\right) !}{\left(-\frac{1}{2}\right) !} \frac{\zeta^{* k} \zeta^{l}}{\left(1-\zeta \zeta^{*}\right)^{l}} \mathrm{P}_{k}^{\left(-\frac{1}{2}, l-k\right)}\left(\frac{1+\zeta \zeta^{*}}{1-\zeta \zeta^{*}}\right) \\
& =(-2)^{k+l} \frac{1 !\left(k-\frac{1}{2}\right) !}{\left(-\frac{1}{2}\right) !} \frac{\zeta^{* k} \zeta^{l}}{\left(1-\zeta \zeta^{*}\right)^{k}} \mathrm{P}_{l}^{\left(-\frac{1}{2}, k-l\right)}\left(\frac{1+\zeta \zeta^{*}}{1-\zeta \zeta^{*}}\right),
\end{aligned}
$$

or alternatively

$$
\begin{aligned}
\overline{a^{\dagger 2 k} a^{2 l}} & =(-2)^{k+l} \frac{k !\left(l-\frac{1}{2}\right) !}{\left(-\frac{1}{2}\right) !} \frac{\zeta^{* k} \zeta^{l}}{\left(1-\zeta \zeta^{*}\right)^{k+l}} \mathrm{P}_{k}^{\left(-\frac{1}{2},-k-l-\frac{1}{2}\right)}\left(1-2 \zeta \zeta^{*}\right) \\
& =(-2)^{k+l} \frac{l !\left(k-\frac{1}{2}\right) !}{\left(-\frac{1}{2}\right) !} \frac{\zeta^{* k} \zeta^{l}}{\left(1-\zeta \zeta^{*}\right)^{k+l}} \mathrm{P}_{l}^{\left(-\frac{1}{2},-k-l-\frac{1}{2}\right)}\left(1-2 \zeta \zeta^{*}\right) .
\end{aligned}
$$

In case of the expectation values $\overline{a^{\dagger 2 k+1} a^{2 l+1}}$ the analogous intermediate result to $(6.1)$ is

$$
\overline{a^{\dagger 2 k+1} a^{2 l+1}}=\sqrt{\frac{1-\zeta \zeta^{*}}{\pi}}(-1)^{k+l} 2^{k+l+1} \zeta^{* k} \zeta^{l} \sum_{j=1}^{\infty} \frac{\left(j+k-\frac{1}{2}\right) !\left(j+l-\frac{1}{2}\right) !}{\left(j-\frac{1}{2}\right) !(j-1) !}\left(\zeta \zeta^{*}\right)^{j} .
$$

Using the relation to the Hypergeometric function and the Jacobi polynomials from this follows in analogy to (6.7)

$$
\begin{aligned}
\overline{a^{\dagger 2 k+1} a^{2 l+1}} & \left.=(-2)^{k+l} \frac{k !\left(l+\frac{1}{2}\right) !}{\left(\frac{1}{2}\right) !} \frac{\zeta^{* k+1} \zeta^{l+1}}{\left(1-\zeta \zeta^{*}\right)^{l+1}} \mathrm{P}_{k}^{\left(\frac{1}{2}, l-k\right.}\right)\left(\frac{1+\zeta \zeta^{*}}{1-\zeta \zeta^{*}}\right) \\
& =(-2)^{k+l} \frac{l !\left(k+\frac{1}{2}\right) !}{\left(\frac{1}{2}\right) !} \frac{\zeta^{* k+1} \zeta^{l+1}}{\left(1-\zeta \zeta^{*}\right)^{k+1}} \mathrm{P}_{l}^{\left(\frac{1}{2}, k-l\right)}\left(\frac{1+\zeta \zeta^{*}}{1-\zeta \zeta^{*}}\right),
\end{aligned}
$$

or, alternatively, in analogy to (6.8) 


$$
\begin{aligned}
\overline{a^{\dagger 2 k+1} a^{2 l+1}} & =(-2)^{k+l} \frac{k !\left(l+\frac{1}{2}\right) !}{\left(\frac{1}{2}\right) !} \frac{\zeta^{* k+1} \zeta^{l+1}}{\left(1-\zeta \zeta^{*}\right)^{k+l+1}} \mathrm{P}_{k}^{\left(\frac{1}{2},-k-l-\frac{3}{2}\right)}\left(1-2 \zeta \zeta^{*}\right) \\
& =(-2)^{k+l} \frac{l !\left(k+\frac{1}{2}\right) !}{\left(\frac{1}{2}\right) !} \frac{\zeta^{* k+1} \zeta^{l+1}}{\left(1-\zeta \zeta^{*}\right)^{k+l+1}} \mathrm{P}_{l}^{\left(\frac{1}{2},-k-l-\frac{3}{2}\right)}\left(1-2 \zeta \zeta^{*}\right) .
\end{aligned}
$$

We checked the special cases $k=l$ in comparison with the representation (5.2) by the Legendre polynomials. We checked too that the right-hand sides of this formula gives for all the four different representations the same result.

There is yet an interesting mathematical aspect. In (5.2) Section 5 we calculated the expectation values $\overline{a^{\dagger k} a^{l}}$ in the special case $k=l$ by formulae which involve Jacobi polynomials (or their special case Legendre polynomials) without distinction of even and odd $k$, whereas in present Section we calculated the more general cases $k \neq l$ and had to distinguish the cases of even $k$ and $l$ and of odd $k$ and $l$ and in the specialization $k=l$ there are involved Jacobi polynomials which are different from that for $k=l$ and it is not possible (or simple) to join these polynomials for $k=l$ to one common formula. The manifold of different transformation relations for Jacobi polynomials is very astonishing (see Appendix C and [27]).

As alternative to the calculation of expectation values by the number representation of squeezed vacuum states one may calculate them from the quasiprobabilities that, however, is also not very simple. With the Wigner quasiprobability $W\left(\alpha, \alpha^{*}\right)$ one may calculate basically the expectation values of symmetrically (Weyl) ordered operators, for example, by integration over the function $\alpha \alpha^{*}$ for the expectation value of the operator $\frac{1}{2}\left(a a^{\dagger}+a^{\dagger} a\right)=N+\frac{1}{2} I$. With the Husimi-Kano quasiprobability $Q\left(\alpha, \alpha^{*}\right)$ one may calculate basically the expectation values of anti-normally operators which one has then to transform to the more interesting expectation values of normally ordered operators. The expectation values of $a^{\dagger k}$ and of $a^{l}$ can be calculated with an arbitrary quasiprobability with the parameter $r$ considered in Section 4 .

\section{Displaced Squeezed Vacuum States or Squeezed Coherent States in Non-Unitary Approach}

As a generalization of squeezed vacuum states we derive here shortly their representation in the basis of number state and discuss a very interesting aspect. It is difficult to deal with squeezing in full generality and one may find in literature many approaches which are special ones (squeezing only in directions of coordinate axes $(q, p))$ or with absent calculation of basic functions connected with them.

We define displaced squeezed vacuum states in the non-unitary approach by applying the displacement operator $D\left(\beta, \beta^{*}\right)$ to squeezed vacuum states 
$|0, \zeta\rangle$ according to

$$
\begin{aligned}
& |\beta, \zeta\rangle \equiv D\left(\beta, \beta^{*}\right)|0, \zeta\rangle=D\left(\beta, \beta^{*}\right) S\left(\zeta^{\prime *}, 0, \zeta^{\prime}\right)|0\rangle, \\
& \left(\zeta^{\prime}, \zeta^{\prime *}\right)=\frac{\operatorname{Arth}(|\zeta|)}{|\zeta|}\left(\zeta, \zeta^{*}\right)
\end{aligned}
$$

where $S\left(\zeta^{\prime *}, 0, \zeta^{\prime}\right)$ is the squeezing operator in unitary approach. We may change the order of operations of displacement and squeezing where the squeezing operator remains the stable part and the displacement operator has to be change. The basic relations for this provides the fundamental representation (A.7) setting there $\eta=0$ and substituting $\zeta \rightarrow-\zeta^{\prime}$

$$
\begin{aligned}
& \left(S\left(\zeta^{\prime^{*}}, 0, \zeta^{\prime}\right)\right)^{\dagger}\left(a, a^{\dagger}\right) S\left(\zeta^{\prime^{*}}, 0, \zeta^{\prime}\right) \\
& =S\left(-\zeta^{\prime^{*}}, 0,-\zeta^{\prime}\right)\left(a, a^{\dagger}\right)\left(S\left(-\zeta^{\prime^{*}}, 0,-\zeta^{\prime}\right)\right)^{\dagger} \\
& =\left(a, a^{\dagger}\right)\left(\begin{array}{cc}
\frac{1}{\sqrt{1-|\zeta|^{2}}}, & -\frac{\zeta^{*}}{\sqrt{1-|\zeta|^{2}}} \\
-\frac{\zeta}{\sqrt{1-|\zeta|^{2}}}, & \frac{1}{\sqrt{1-|\zeta|^{2}}}
\end{array}\right) .
\end{aligned}
$$

These transformations of $\left(a, a^{\dagger}\right)$ after transition to the Hermitean basis of operators $(Q, P)$ are very similar to Special Lorentz transformations of $(x, c t)$ with $x$ one space coordinate and $t$ the time and this is not incidental since it is for real parameter $\zeta$ the same one-parameter Lie group. From (7.2) follows

$$
\begin{aligned}
& \left(S\left(\zeta^{\prime^{*}}, 0, \zeta^{\prime}\right)\right)^{\dagger} D\left(\beta, \beta^{*}\right) S\left(\zeta^{\prime^{*}}, 0, \zeta^{\prime}\right) \\
& =\left(S\left(\zeta^{\prime^{*}}, 0, \zeta^{\prime}\right)\right)^{\dagger} \exp \left(\beta a^{\dagger}-\beta^{*} a\right) S\left(\zeta^{\prime^{*}}, 0, \zeta^{\prime}\right) \\
& =\exp \left(\beta \frac{a^{\dagger}-\zeta^{*} a}{\sqrt{1-|\zeta|^{2}}}-\beta^{*} \frac{a-\zeta a^{\dagger}}{\sqrt{1-|\zeta|^{2}}}\right) \\
& =\exp \left(\frac{\beta+\zeta \beta^{*}}{\sqrt{1-|\zeta|^{2}}} a^{\dagger}-\frac{\beta^{*}+\zeta^{*} \beta}{\sqrt{1-|\zeta|^{2}}} a\right) \\
& =D\left(\frac{\beta+\zeta \beta^{*}}{\sqrt{1-|\zeta|^{2}}}, \frac{\beta^{*}+\zeta^{*} \beta}{\sqrt{1-|\zeta|^{2}}}\right) .
\end{aligned}
$$

Applied to (7.1) we find (see also Schleich [13], p. 125)

$$
\begin{aligned}
& |\beta, \zeta\rangle=D\left(\beta, \beta^{*}\right)|0, \zeta\rangle=D\left(\beta, \beta^{*}\right) S\left(\zeta^{\prime *}, 0, \zeta^{\prime}\right)|0\rangle \\
& =S\left(\zeta^{\prime^{*}}, 0, \zeta^{\prime}\right) D\left(\frac{\beta+\zeta \beta^{*}}{\sqrt{1-|\zeta|^{2}}}, \frac{\beta^{*}+\zeta^{*} \beta}{\sqrt{1-|\zeta|^{2}}}\right)|0\rangle \\
& =S\left(\zeta^{\prime *}, 0, \zeta^{\prime}\right)\left|\beta^{\prime}, 0\right\rangle, \quad\left(\beta^{\prime}, \beta^{\prime^{*}}\right) \equiv\left(\frac{\beta+\zeta \beta^{*}}{\sqrt{1-|\zeta|^{2}}}, \frac{\beta^{*}+\zeta^{*} \beta}{\sqrt{1-|\zeta|^{2}}}\right),
\end{aligned}
$$


as alternative representation of squeezed vacuum states. This means that we make first a displacement of the vacuum state $|0\rangle$ to a coherent state $\left|\beta^{\prime}, 0\right\rangle$ with the changed displacement parameter $\beta^{\prime}$ and after this the squeezing of the coherent state with the same squeezing operator $S\left(\zeta^{\prime *}, 0, \zeta^{\prime}\right)$ as in the first variant. Therefore, displaced squeezed vacuum states are fully equivalent to squeezed coherent states with the changed displacement parameters as seen from (7.1) and (7.4). For $\zeta=0$ one obtains from $|\beta, \zeta\rangle$ the coherent states and for $\beta=0$ the squeezed vacuum states

$$
\begin{aligned}
& |\beta, 0\rangle=D\left(\beta, \beta^{*}\right)|0,0\rangle \equiv|\beta\rangle, \\
& |0, \zeta\rangle=S\left(\zeta^{\prime^{*}}, 0, \zeta^{\prime}\right)|0,0\rangle, \quad|0,0\rangle \equiv|0\rangle .
\end{aligned}
$$

The squeezing operator is the stable part in these two alternative representations. Figure 3 shows schematically the displacement of squeezed vacuum states under fixed complex squeezing parameters $\zeta$ in different directions of the complex phase plane described by the complex parameter $\beta$ $(|\beta|=$ const in the four particular pictures).

In generalization of the well-known eigenvalue equation of coherent states $|\beta, 0\rangle \equiv|\beta\rangle$ the displaced squeezed vacuum states $|\beta, \zeta\rangle$ are right-hand eigenstates of the operator $a+\zeta a^{\dagger}$ to eigenvalues $\beta+\zeta \beta^{*}$ according to

$$
\left(a+\zeta a^{\dagger}\right)|\beta, \zeta\rangle=\left(\beta+\zeta \beta^{*}\right)|\beta, \zeta\rangle \text {. }
$$

This follows from the relation

$$
\begin{aligned}
& \left(a+\zeta a^{\dagger}\right) \exp \left(\beta a^{\dagger}-\beta^{*} a\right)|0, \zeta\rangle \\
& =\exp \left(\beta a^{\dagger}-\beta^{*} a\right)\left(a+\beta I+\zeta\left(a^{\dagger}+\beta^{*} I\right)\right)|0, \zeta\rangle \\
& =\left(\beta+\zeta \beta^{*}\right) \exp \left(\beta a^{\dagger}-\beta^{*} a\right)|0, \zeta\rangle,
\end{aligned}
$$

using that $|0, \zeta\rangle$ are right-hand eigenstates of $a+\zeta a^{\dagger}$ to eigenvalue zero (see (2.12)).

We now derive the number representation of displaced squeezed vacuum states. Using the normally order form (2.7) of the squeezing operator and the following normally ordered form of the displacement operator

$$
D\left(\beta, \beta^{*}\right)=\exp \left(-\frac{\beta \beta^{*}}{2}\right) \exp \left(\beta a^{\dagger}\right) \exp \left(-\beta^{*} a\right),
$$

from the definition (7.1) follows

$$
\begin{aligned}
& |\beta, \zeta\rangle \\
& =\exp \left(-\frac{\beta \beta^{*}}{2}\right)\left(1-\zeta \zeta^{*}\right)^{\frac{1}{4}} \exp \left(\beta a^{\dagger}\right) \exp \left(-\beta^{*} a\right) \exp \left(-\frac{\zeta}{2} a^{\dagger 2}\right)|0\rangle \\
& =\left(1-\zeta \zeta^{*}\right)^{\frac{1}{4}} \exp \left(-\frac{\beta \beta^{*}}{2}\right) \exp \left(\beta a^{\dagger}\right) \exp \left(-\frac{\zeta}{2}\left(a^{\dagger}-\beta^{*} I\right)^{2}\right) \exp \left(-\beta^{*} a\right)|0\rangle \\
& =\left(1-\zeta \zeta^{*}\right)^{\frac{1}{4}} \exp \left(-\frac{\beta \beta^{*}+\zeta \beta^{* 2}}{2}\right) \exp \left(\left(\beta+\zeta \beta^{*}\right) a^{\dagger}-\frac{\zeta}{2} a^{\dagger 2}\right)|0\rangle
\end{aligned}
$$


Squeezing ellipses to different displacements, $\zeta=+|\zeta|$

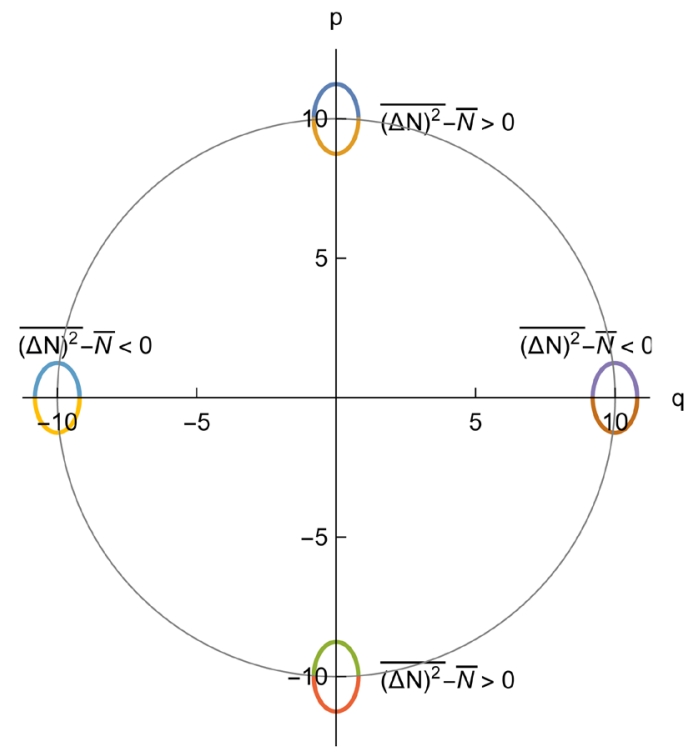

Squeezing ellipses to different displacements, $\zeta=-|\zeta|$

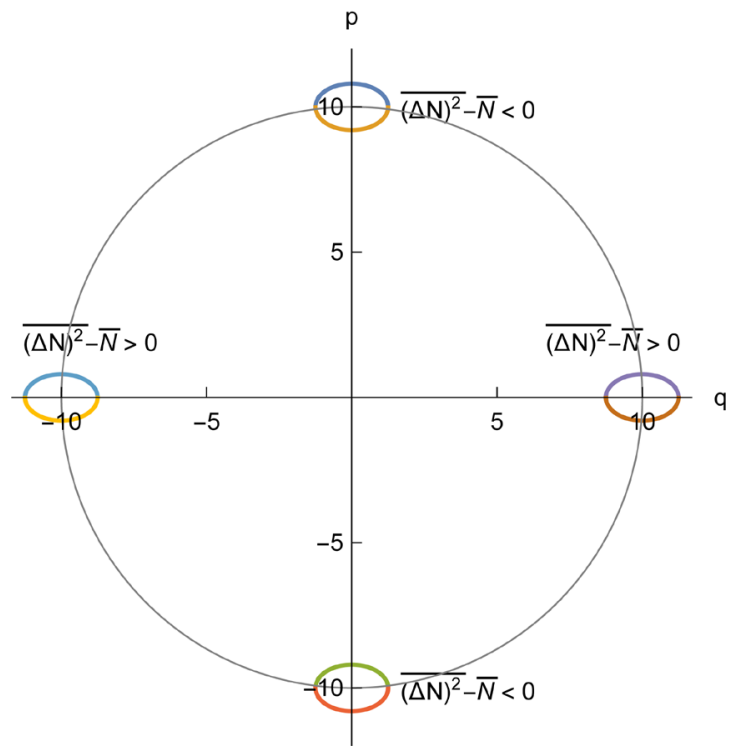

Squeezing ellipses to different displacements, $\zeta=+i|\zeta|$

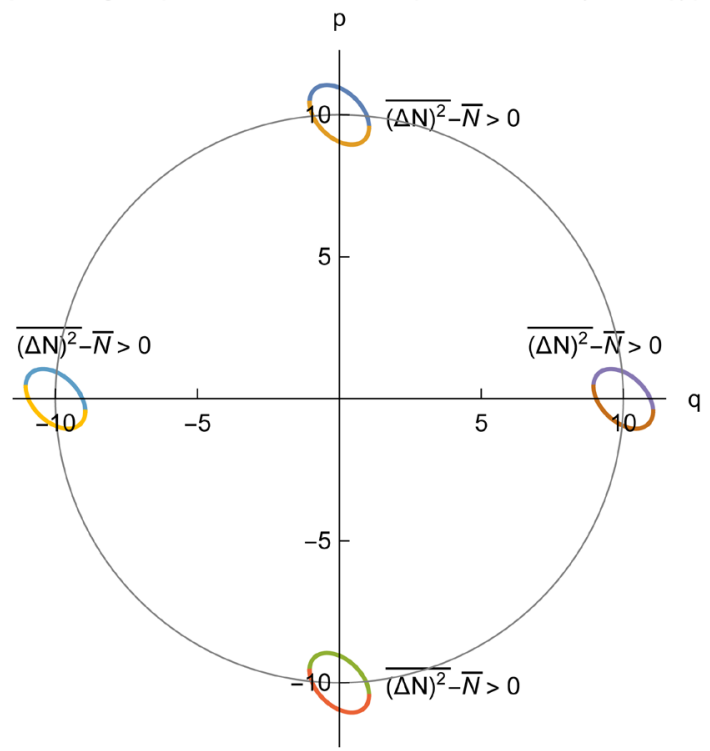

Squeezing ellipses to different displacements, $\zeta=-i|\zeta|$

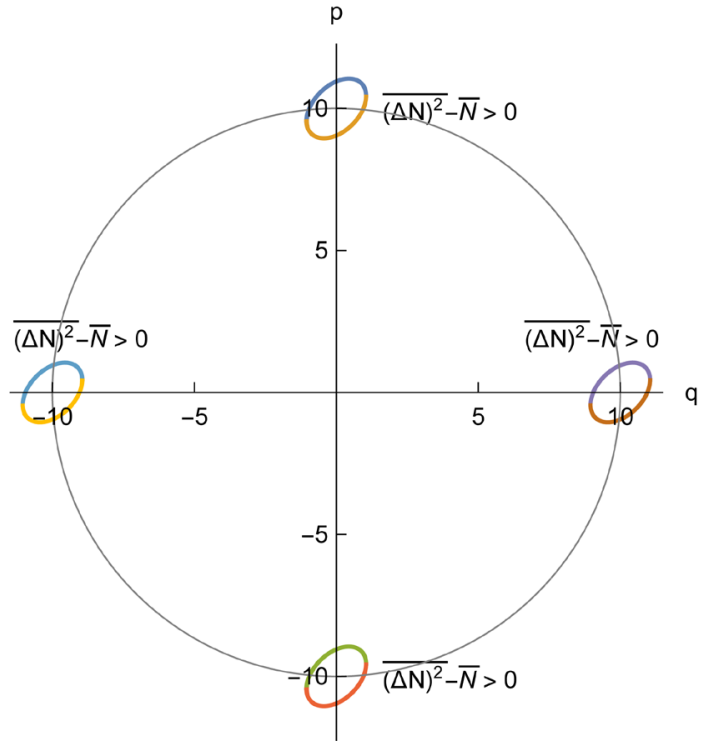

Figure 3. Squeezing ellipses in relation to the displacement parameter of squeezed coherent states (schematically). The mean value $|\bar{N}|$ of the number operator $N$ depends for squeezed coherent states only on the modulus $|\zeta|$ of the squeezing parameter $\zeta$ left constant here and on the modulus $|\beta|$ of the complex displacement parameter $\beta$ and, therefore, is the same on the shown circles in all four partial figures. The case of squeezing in direction of the displacement parameter $\beta$ is also called "amplitude squeezing" (in $q$-direction in first figure) and in opposite direction to the displacement parameter $\beta$ "phase squeezing" (in p-direction in first figure).

If we now apply the generating function (D.1) for Hermite polynomials to the factor in front of $|0\rangle$ we obtain the following form of the representation of displaced squeezed vacuum states

$$
|\beta, \zeta\rangle=\left(1-\zeta \zeta^{*}\right)^{\frac{1}{4}} \exp \left(-\frac{\beta \beta^{*}+\zeta \beta^{* 2}}{2}\right) \sum_{n=0}^{\infty} \frac{1}{n !}\left(\frac{\sqrt{2 \zeta}}{2} a^{\dagger}\right)^{n} \mathrm{H}_{n}\left(\frac{\beta+\zeta \beta^{*}}{\sqrt{2 \zeta}}\right)|0\rangle,
$$


and using the generation of number states $|n\rangle$ from the vacuum state $|0\rangle$ (see (2.1)) one finds the final form of the number-state representation ${ }^{2}$

$$
|\beta, \zeta\rangle=\left(1-\zeta \zeta^{*}\right)^{\frac{1}{4}} \exp \left(-\frac{\left(\beta+\zeta \beta^{*}\right) \beta^{*}}{2}\right) \sum_{n=0}^{\infty} \frac{1}{\sqrt{n !}}\left(\frac{\sqrt{2 \zeta}}{2}\right)^{n} \mathrm{H}_{n}\left(\frac{\beta+\zeta \beta^{*}}{\sqrt{2 \zeta}}\right)|n\rangle \text {. }
$$

It is easy to see that for $\beta=0$ using $\mathrm{H}_{2 m}(0)=\frac{(-1)^{m}(2 m) !}{m !}, \mathrm{H}_{2 m+1}(0)=0$ one obtains the number representation (2.3) of squeezed vacuum states $|0, \zeta\rangle$ and for $\zeta=0$ using $\lim _{z \rightarrow \infty} \frac{1}{(2 z)^{n}} H_{n}(z)=1$ the number representation of coherent states $|\beta\rangle$.

From (7.10) one finds the probabilities $p_{n}$ of the photon statistics

$$
\begin{aligned}
p_{n} \equiv & \langle n \mid \beta, \zeta\rangle\langle\beta, \zeta \mid n\rangle \\
= & \sqrt{1-\zeta \zeta^{*}} \exp \left(-\frac{2 \beta \beta^{*}+\zeta^{*} \beta^{2}+\zeta \beta^{* 2}}{2}\right) \frac{1}{n !}\left(\frac{\sqrt{\zeta \zeta^{*}}}{2}\right)^{n} \\
& \times \mathrm{H}_{n}\left(\frac{\beta+\zeta \beta^{*}}{\sqrt{2 \zeta}}\right) \mathrm{H}_{n}\left(\frac{\beta^{*}+\zeta^{*} \beta}{\sqrt{2 \zeta^{*}}}\right) .
\end{aligned}
$$

By means of the generating function (D.2) for products of two Hermite polynomials it can be affirmed that the states $|\beta, \zeta\rangle$ are normalized and that the probabilities $p_{n}$ satisfy the necessary relation

$$
\sum_{n=0}^{\infty} p_{n}=1
$$

In contrast to the photon distributions of coherent states $|\beta\rangle \equiv|\beta, 0\rangle$ which depends only on $|\beta|^{2} \equiv \beta \beta^{*}$ and of squeezed vacuum states $|0, \zeta\rangle$ which depends only on $|\zeta|^{2} \equiv \zeta \zeta^{*}<1$ the photon distribution (7.12) depends in addition to the moduli also on the phases of $\zeta$ and $\beta$ in the complex plane.

The nearest coherent state to the state $|\beta, \zeta\rangle$ is the state $|\beta\rangle$ and for this distance $d(|\beta, \zeta\rangle\langle\beta, \zeta|,| \beta\rangle\langle\beta|)$ one obtains the same value as on the right-hand side of (2.16). This means that it does not depend on $\beta$.

\section{Wave Functions of Displaced Squeezed Vacuum States or Squeezed Coherent States}

As important characteristics of the displaced squeezed vacuum states $|\beta, \zeta\rangle$ we now calculate their wave functions $\langle q \mid \beta, \zeta\rangle$ and $\langle p \mid \beta, \zeta\rangle$ in the eigenstates $|q\rangle$ and $|p\rangle$ of the canonical operators $Q$ and $P$ (in the usual standardizations $\left\langle q \mid q^{\prime}\right\rangle=\delta\left(q-q^{\prime}\right), \int_{-\infty}^{+\infty} \mathrm{d} q|q\rangle\langle q|=I$ and similar for $\left.|p\rangle\right)$. From the number representation (7.11) of the states and the well-known number representation of $|q\rangle$ and $|p\rangle$ follows as the first step in the calculation

${ }^{2}$ In both parts with $\sqrt{2 \zeta}$ one has to choose the same sign of the root but which sign does not matter. 


$$
\begin{aligned}
\langle q \mid \beta, \zeta\rangle= & \left(\frac{1-\zeta \zeta^{*}}{\hbar \pi}\right)^{\frac{1}{4}} \exp \left(-\frac{q^{2}}{2 \hbar}-\frac{\beta^{*}\left(\beta+\zeta \beta^{*}\right)}{2}\right) \\
& \times \sum_{n=0}^{\infty} \frac{(\sqrt{\zeta})^{n}}{2^{n} n !} \mathrm{H}_{n}\left(\frac{q}{\sqrt{\hbar}}\right) \mathrm{H}_{n}\left(\frac{\beta+\zeta \beta^{*}}{\sqrt{2 \zeta}),}\right. \\
\langle p \mid \beta, \zeta\rangle= & \left(\frac{1-\zeta \zeta^{*}}{\hbar \pi}\right)^{\frac{1}{4}} \exp \left(-\frac{p^{2}}{2 \hbar}-\frac{\beta^{*}\left(\beta+\zeta \beta^{*}\right)}{2}\right) \\
& \times \sum_{n=0}^{\infty} \frac{(-\mathrm{i} \sqrt{\zeta})^{n}}{2^{n} n !} \mathrm{H}_{n}\left(\frac{p}{\sqrt{\hbar}}\right) \mathrm{H}_{n}\left(\frac{\beta+\zeta \beta^{*}}{\sqrt{2 \zeta}}\right) .
\end{aligned}
$$

The infinite sums can be calculated in closed form using the generating function (D.2) for the product of two Hermite polynomials. We represent the result by the mean values $\bar{Q} \equiv\langle\beta, \zeta|Q| \beta, \zeta\rangle$ and $\bar{P} \equiv\langle\beta, \zeta|P| \beta, \zeta\rangle$ of the canonical operators $Q$ and $P$. For squeezed vacuum states $|0, \zeta\rangle$ these mean values vanish already due to the symmetry and for the displaced squeezed vacuum states they are simply connected with the complex displacement parameter $\left(\beta, \beta^{*}\right)$ according to

$$
\bar{Q}=\sqrt{\frac{\hbar}{2}}\left(\beta+\beta^{*}\right), \quad \bar{P}=-\mathrm{i} \sqrt{\frac{\hbar}{2}}\left(\beta-\beta^{*}\right) .
$$

With these parameters the result of the evaluation of the sums in (8.1) can be represented in the form

$$
\begin{aligned}
& \langle q \mid \beta, \zeta\rangle \\
= & \left(\frac{1-\zeta \zeta^{*}}{\hbar \pi}\right)^{\frac{1}{4}} \frac{1}{\sqrt{1-\zeta}} \exp \left(\mathrm{i} \frac{\bar{P}(2 q-\bar{Q})}{2 \hbar}\right) \exp \left\{-\frac{(1+\zeta)(q-\bar{Q})^{2}}{2(1-\zeta) \hbar}\right\}, \\
\langle p \mid \beta, \zeta\rangle & \left(\frac{1-\zeta \zeta^{*}}{\hbar \pi}\right)^{\frac{1}{4}} \frac{1}{\sqrt{1+\zeta}} \exp \left(-\mathrm{i} \frac{\bar{Q}(2 p-\bar{P})}{2 \hbar}\right) \exp \left\{-\frac{(1-\zeta)(p-\bar{P})^{2}}{2(1+\zeta) \hbar}\right\} .
\end{aligned}
$$

One may make cross checks of these relations using the pair of Fourier transformations

$$
\begin{aligned}
& \langle p \mid \beta, \zeta\rangle=\frac{1}{\sqrt{2 \hbar \pi}} \int_{-\infty}^{+\infty} \mathrm{d} q \exp \left(-\mathrm{i} \frac{p q}{\hbar}\right)\langle q \mid \beta, \zeta\rangle, \\
& \langle q \mid \beta, \zeta\rangle=\frac{1}{\sqrt{2 \hbar \pi}} \int_{-\infty}^{+\infty} \mathrm{d} p \exp \left(\mathrm{i} \frac{q p}{\hbar}\right)\langle p \mid \beta, \zeta\rangle .
\end{aligned}
$$

From (8.3) one finds the Gaussian distributions

$$
\begin{aligned}
W(q) & \equiv\langle q \mid \beta, \zeta\rangle\langle\beta, \zeta \mid q\rangle \\
& =\sqrt{\frac{1-\zeta \zeta^{*}}{(1-\zeta)\left(1-\zeta^{*}\right) \hbar \pi}} \exp \left\{-\frac{1-\zeta \zeta^{*}}{(1-\zeta)\left(1-\zeta^{*}\right)} \frac{(q-\bar{Q})^{2}}{\hbar}\right\},
\end{aligned}
$$




$$
\begin{aligned}
W(p) & \equiv\langle p \mid \beta, \zeta\rangle\langle\beta, \zeta \mid p\rangle \\
& =\sqrt{\frac{1-\zeta \zeta^{*}}{(1+\zeta)\left(1+\zeta^{*}\right) \hbar \pi}} \exp \left\{-\frac{1-\zeta \zeta^{*}}{(1+\zeta)\left(1+\zeta^{*}\right)} \frac{(p-\bar{P})^{2}}{\hbar}\right\},
\end{aligned}
$$

with the normalization

$$
\int_{-\infty}^{+\infty} \mathrm{d} q W(q)=\int_{-\infty}^{+\infty} \mathrm{d} p W(p)=1 .
$$

The functions $W(q)$ and $W(p)$ are equal to the Wigner quasiprobability $W(q, p)$ integrated over one of the canonical variables $p$ or $q$ and nothing speaks as known against an interpretation of genuine one-dimensional probability densities. They remain invariant with interchanging $\zeta \leftrightarrow \zeta^{*}$ and the states are not uniquely reconstructible from $W(q)$ and $W(p)$ alone. In our case of (8.5) they are one-dimensional normalized Gaussian distributions around $\bar{Q}$ and $\bar{P}$, respectively, with the variances of $\bar{Q}$ and $\bar{P}$

$$
\begin{aligned}
& \overline{(\Delta Q)^{2}}=\frac{(1-\zeta)\left(1-\zeta^{*}\right)}{1-\zeta \zeta^{*}} \frac{\hbar}{2}, \\
& \overline{(\Delta P)^{2}}=\frac{(1+\zeta)\left(1+\zeta^{*}\right)}{1-\zeta \zeta^{*}} \frac{\hbar}{2},
\end{aligned}
$$

The uncertainty product (see also (3.7))

$$
\overline{(\Delta Q)^{2}} \overline{(\Delta P)^{2}}=\frac{\left(1-\zeta^{2}\right)\left(1-\zeta^{* 2}\right)}{\left(1-\zeta \zeta^{*}\right)^{2}} \frac{\hbar^{2}}{4} \geq \frac{\hbar^{2}}{4},
$$

is only for real squeezing parameter $\zeta$ but not for complex $\zeta$ the minimal possible one.

In case of real squeezing parameter $\zeta=\zeta^{*}$ one obtains from (8.3)

$$
\begin{aligned}
& \langle q \mid \beta, \zeta\rangle=\frac{1}{\left(2 \sqrt{\overline{(\Delta Q)^{2}}} \pi\right)^{\frac{1}{4}}} \exp \left(\mathrm{i} \frac{\bar{P}(2 q-\bar{Q})}{2 \hbar}\right) \exp \left\{-\frac{(q-\bar{Q})^{2}}{4(\Delta Q)^{2}}\right\}, \\
& \langle p \mid \beta, \zeta\rangle=\frac{1}{\left(2 \sqrt{(\Delta P)^{2}} \pi\right)^{\frac{1}{4}}} \exp \left(-\mathrm{i} \frac{\bar{Q}(2 p-\bar{P})}{2 \hbar}\right) \exp \left\{-\frac{(p-\bar{P})^{2}}{4 \overline{(\Delta P)^{2}}}\right\},
\end{aligned}
$$

with the variances of $Q$ and $P$

$$
\overline{(\Delta Q)^{2}}=\frac{1-\zeta}{1+\zeta} \frac{\hbar}{2}, \quad \overline{(\Delta P)^{2}}=\frac{1+\zeta}{1-\zeta} \frac{\hbar}{2}, \quad \overline{(\Delta Q)^{2}} \overline{(\Delta P)^{2}}=\frac{\hbar^{2}}{4}, \quad\left(\zeta=\zeta^{*}\right),
$$

As already explained in Section 3 only in case of real $\zeta=\zeta^{*}$ the squeezing axes coincide with the axes of $(q, p)$ and the uncertainty product becomes the minimal one but taking into account the uncertainty correlation the Gaussian states with other positions of the squeezing axes can be included into the minimum uncertainty states (see Section 3). 
By differentiation of relations (8.9) with respect to variables $q$ and $p$ follows

$$
\begin{aligned}
-\mathrm{i} \hbar\left(\frac{\partial}{\partial q}+\frac{q}{2(\Delta Q)^{2}}\right)\langle q \mid \beta, \zeta\rangle & \equiv\left\langle q\left|\left(P-\frac{\mathrm{i} \hbar}{2(\Delta Q)^{2}} Q\right)\right| \beta, \zeta\right\rangle \\
& =\left(\bar{P}-\frac{\mathrm{i} \hbar \bar{Q}}{2\left(\overline{Q Q)^{2}}\right.}\right)\langle q \mid \beta, \zeta\rangle, \\
\mathrm{i} \hbar\left(\frac{\partial}{\partial p}+\frac{p}{2(\Delta P)^{2}}\right)\langle p \mid \beta, \zeta\rangle & \equiv\left\langle p\left|\left(Q+\frac{\mathrm{i} \hbar}{2(\Delta Q)^{2}} P\right)\right| \beta, \zeta\right\rangle \\
& =\left(\bar{Q}+\frac{\mathrm{i} \hbar \bar{P}}{2\left(\overline{(\Delta P)^{2}}\right)\langle p \mid \beta, \zeta\rangle,}\right.
\end{aligned}
$$

where we used $\langle q| P=-\mathrm{i} \hbar \frac{\partial}{\partial q}\langle q|$ and $\langle p| Q=\mathrm{i} \hbar \frac{\partial}{\partial p}\langle p|$. The normalization of the states in (8.10) and the pure phase factors $\exp \left(\mp \mathrm{i} \frac{\bar{P} \bar{Q}}{2 \hbar}\right)$ do not follow from the differential equations (8.11) and must be determined where the mentioned phase factors must be present to get full agreement with the usual definitions of the phases of the states $|q\rangle$ and $|p\rangle$.

Pauli in his hand-book article [2] (pp. 20, 21) derives the first of the differential equations (8.11), however, with the right-hand side equal to zero that means he does not take into account the possible displacements $\bar{Q}$ and $\bar{P}$ that corresponds to squeezed vacuum states (displaced squeezed vacuum states were not known or discussed at that time but Schrödinger considered already coherent states although not under this name in [1]). More general and complete are the derivations in the monographs of Louisell [3] (p. 50, Equation (1.12.23)) and of Leonhardt [14] (p. 32, Equation (2.85)). The derivations of Pauli are the usual ones in the theory of elements (states) and operators in a Hilbert space known already at that time. Starting from the axiom of positive definiteness of the norm (square root of scalar product of an element with itself) of non-zero elements they consider the superpositions of two arbitrary states and from their norms they derive first the Cauchy-Bunyakovski-Schwarz inequality and second from the vanishing of the superpositions of the two states that they have to be linearly dependent [33]. In the derivations of uncertainty relations this is applied to two states which are generated from one state $|\psi\rangle$ by applying two different operators to this one state (here of $Q-\bar{Q} I$ and $P-\bar{P} I$ ). For the minimum of the norm (equal to zero) this superposition has to be linearly dependent as seen in (8.11). This leads then to the superposition of the two states $(Q-\bar{Q} I)|\beta, \zeta\rangle$ and $(P-\bar{P} I)|\beta, \zeta\rangle$ (see also (2.13)) with real $\zeta$ and $\beta$ in which Pauli in foresight of the result introduced $(\Delta Q)^{2}$ instead of a more indifferent real parameter $\lambda$. Thus the result is connected with the basic assumption that all states in quantum optics may be considered as states with positive norm in a Hilbert space. 
The displaced squeezed vacuum states or squeezed coherent states $|\beta, \zeta\rangle$ are the most general pure states with Gaussian distributions.

\section{Bargmann Representation and Wigner Quasiprobability for Displaced Squeezed Vacuum States}

The Bargmann representation of states is the representation by an analytic function of $\alpha$ obtain by forming the scalar product of the state with the analytic but non-normalized coherent state $\| \alpha\rangle \equiv \sum_{n=0}^{\infty} \frac{\alpha^{n}}{\sqrt{n !}}|0\rangle$ (see Section 4). From the number representation of displaced squeezed vacuum states (7.11) using the generating function for Hermite polynomials (9.1) we find

$$
\begin{aligned}
\exp \left(\frac{\alpha \alpha^{*}}{2}\right)\langle 0, \zeta \mid \alpha\rangle & =\left(1-\zeta \zeta^{*}\right)^{\frac{1}{4}} \exp \left(-\frac{\beta \beta^{*}}{2}+\alpha \beta^{*}-\frac{\zeta^{*}}{2}(\alpha-\beta)^{2}\right) \\
& =\left(\exp \left(\frac{\alpha \alpha^{*}}{2}\right)\langle\alpha \mid 0, \zeta\rangle\right)^{*}
\end{aligned}
$$

The Bargmann representation of a state contains the full information about the state. From (9.1) one finds the Husimi-Kano quasiprobability

$$
\begin{aligned}
& Q\left(\alpha, \alpha^{*}\right) \equiv \frac{\langle\alpha \mid \beta, \zeta\rangle\langle\beta, \zeta \mid \alpha\rangle}{\pi} \\
& =\frac{\sqrt{1-\zeta \zeta^{*}}}{\pi} \exp \left\{-\left((\alpha-\beta)\left(\alpha^{*}-\beta^{*}\right)+\frac{\zeta^{*}}{2}(\alpha-\beta)^{2}+\frac{\zeta}{2}\left(\alpha^{*}-\beta^{*}\right)^{2}\right)\right\}, \\
& \int \frac{\mathrm{i}}{2} \mathrm{~d} \alpha \wedge \mathrm{d} \alpha^{*} Q\left(\alpha, \alpha^{*}\right)=1 .
\end{aligned}
$$

This is the corresponding Husimi-Kano quasiprobability (4.2) for squeezed vacuum states with argument displacement.

In Appendix B it is shown by a very simple transformation that the Wigner quasiprobability $W\left(\alpha, \alpha^{*}\right)$ of a displaced state can be obtained from Wigner quasiprobability $W_{0}\left(\alpha, \alpha^{*}\right)$ of the corresponding undisplaced state by a simple argument displacement $\left(\alpha, \alpha^{*}\right) \rightarrow\left(\alpha-\beta, \alpha^{*}-\beta^{*}\right)$. This is also true for other quasiprobabilities such as for example the Husimi-Kano quasiprobability $Q\left(\alpha, \alpha^{*}\right)$ as we saw and for the Glauber-Sudarshan quasiprobability $P\left(\alpha, \alpha^{*}\right)$. In this way one obtains from (4.6) for the Wigner quasiprobability of displaced squeezed vacuum states

$$
\begin{aligned}
& W\left(\alpha, \alpha^{*}\right)=\frac{2}{\pi} \exp \left\{-2 \frac{\left(\alpha-\beta+\zeta\left(\alpha^{*}-\beta^{*}\right)\right)\left(\alpha^{*}-\beta^{*}+\zeta(\alpha-\beta)\right)}{1-\zeta \zeta^{*}}\right\}, \\
& \int \frac{\mathrm{i}}{2} \mathrm{~d} \alpha \wedge \mathrm{d} \alpha^{*} W\left(\alpha, \alpha^{*}\right)=1 .
\end{aligned}
$$

As discussed in Section 7 as alternative we may first make a displacement of the vacuum state to a coherent state with the displacement parameter $\left(\frac{\beta+\zeta \beta^{*}}{\sqrt{1-\zeta \zeta^{*}}}, \frac{\beta^{*}+\zeta^{*} \beta}{\sqrt{1-\zeta \zeta^{*}}}\right)$ that provides the Wigner quasiprobability 


$$
W_{0}\left(\alpha, \alpha^{*}\right)=\frac{2}{\pi} \exp \left\{-2\left(\alpha-\frac{\beta+\zeta \beta^{*}}{\sqrt{1-\zeta \zeta^{*}}}\right)\left(\alpha^{*}-\frac{\beta^{*}+\zeta^{*} \beta}{\sqrt{1-\zeta \zeta^{*}}}\right)\right\}
$$

If we now make the squeezing of coherent state with the same squeezing operator as in the other variant (see (7.4)) we have to transform the arguments in (9.4) according to $\left(\alpha, \alpha^{*}\right) \rightarrow\left(\frac{\alpha+\zeta \alpha^{*}}{\sqrt{1-\zeta \zeta^{*}}}, \frac{\alpha^{*}+\zeta^{*} \alpha}{\sqrt{1-\zeta \zeta^{*}}}\right)$ (see also (4.4)) and we obtain again the Wigner quasiprobability (9.3) that affirms the interchangeability of squeezing and displacement according to (7.4).

The Wigner quasiprobability for displaced squeezed vacuum states in the representation by canonical variables $(q, p)$ can be obtained from (4.7) by the following substitution of the canonical variables $(q, p)$

$$
(q, p) \rightarrow(q-\bar{Q}, p-\bar{P}),\left(\bar{a}, \bar{a}^{*}\right) \equiv\left(\frac{\bar{Q}+\mathrm{i} \bar{P}}{\sqrt{2 \hbar}}, \frac{\bar{Q}-\mathrm{i} \bar{P}}{\sqrt{2 \hbar}}\right)=\left(\beta, \beta^{*}\right),\left(\overline{a^{\dagger}}=\bar{a}^{*}\right) .
$$

We do not write down this. In analogous way we may find the other quasiprobabilities in the representation by canonical variables.

The given quasiprobabilities can be used for the calculation of expectation values for displaced squeezed vacuum states or squeezed coherent states but in next Section we present an alternative for such calculations.

\section{Calculation of Expectation Values for Displaced States from the Expectation Values of the Undisplaced States}

An alternative for the calculation of expectation values of ordered powers of the annihilation and creation operator for displaced states from that for the undisplaced states is the following possibility presented here for normal ordering. Using the unitary displacement operator $D\left(\beta, \beta^{*}\right)$ one finds applied to displaced squeezed vacuum states

$$
\begin{aligned}
\left(\overline{a^{\dagger k} a^{l}}\right)_{\beta} & \equiv\left\langle\beta, \zeta\left|a^{\dagger k} a^{l}\right| \beta, \zeta\right\rangle \\
& =\langle 0, \zeta|\left(D\left(\beta, \beta^{*}\right)^{\dagger} a^{\dagger k} a^{l} D\left(\beta, \beta^{*}\right)|0, \zeta\rangle\right. \\
& =\left\langle 0, \zeta|\left(a^{\dagger}+\beta^{*} I\right)^{k} \underbrace{\left(D\left(\beta, \beta^{*}\right)\right)^{\dagger} D\left(\beta, \beta^{*}\right)}_{=I}(a+\beta I)^{l}| 0, \zeta\right\rangle
\end{aligned}
$$

that after Taylor series expansion of the binomials can be written

$$
\left(\overline{a^{\dagger k} a^{l}}\right)_{\beta}=\sum_{i=0}^{k} \sum_{j=0}^{l} \frac{k ! l !}{i !(k-i) ! j !(l-j) !} \beta^{*_{i}} \beta^{j}\left(\overline{a^{\dagger k-i} a^{l-j}}\right)_{0},
$$

where index 0 at expectation values means the expectation values before the displacement and with index $\beta$ after the displacement with the complex parameter $\beta$.

For squeezed vacuum states the expectation values $\left(\overline{a^{\dagger k} a^{l}}\right)_{0}$ are only nonvanishing if the difference $|k-l|$ is an even number. Taking this into account 
we find for displaced squeezed vacuum states the expectation values of the operators $a$ and $a^{\dagger}$

$$
(\bar{a})_{\beta}=\beta(\bar{I})_{0}=\beta, \quad\left(\overline{a^{\dagger}}\right)_{\beta}=\beta^{*}(\bar{I})_{0}=\beta^{*},
$$

and for the expectation values of the operators $a^{2}$ and $a^{\dagger 2}$

$$
\begin{aligned}
& \left(\overline{a^{2}}\right)_{\beta}=\left(\overline{a^{2}}\right)_{0}+\beta^{2}(\bar{I})_{0}=-\frac{\zeta}{1-\zeta \zeta^{*}}+\beta^{2}, \\
& \left(\overline{a^{\dagger 2}}\right)_{\beta}=\left(\overline{a^{\dagger 2}}\right)_{0}+\beta^{* 2}(\bar{I})_{0}=-\frac{\zeta^{*}}{1-\zeta \zeta^{*}}+\beta^{* 2} .
\end{aligned}
$$

Next we calculate expectation values of operators which are important for the photon statistics. The expectation value of the number operator $N=a^{\dagger} a$ depends only on the squared moduli $|\zeta|^{2} \equiv \zeta \zeta^{*}$ and $|\beta|^{2} \equiv \beta \beta^{*}$ of the complex $\zeta$ and $\beta$ and we find

$$
(\bar{N})_{\beta}=\left(\overline{a^{\dagger} a}\right)_{\beta}=\left(\overline{a^{\dagger} a}\right)_{0}+\beta \beta^{*}(\bar{I})_{0}=\frac{\zeta \zeta^{*}}{1-\zeta \zeta^{*}}+\beta \beta^{*}=\frac{|\zeta|^{2}}{1-|\zeta|^{2}}+|\beta|^{2} .
$$

For the discussion of sub- and super-Poissonian statistics of displaced squeezed vacuum states we need in addition the expectation value of the operator $a^{\dagger 2} a^{2}$. It does not only depend on the moduli of $\zeta$ and $\beta$ but also on their phases and from (10.2) we find

$$
\begin{aligned}
\left(\overline{a^{\dagger 2} a^{2}}\right)_{\beta}= & \left(\overline{a^{\dagger 2} a^{2}}\right)_{0}+4 \beta \beta^{*}\left(\overline{a^{\dagger} a}\right)_{0}+\left(\beta \beta^{*}\right)^{2}(\bar{I})_{0}+\beta^{2}\left(\overline{a^{\dagger 2}}\right)_{0}+\beta^{* 2}\left(\overline{a^{2}}\right)_{0} \\
= & \frac{\zeta \zeta^{*}\left(1+2 \zeta \zeta^{*}\right)}{\left(1-\zeta \zeta^{*}\right)^{2}}+4 \beta \beta^{*} \frac{\zeta \zeta^{*}}{1-\zeta \zeta^{*}}+\left(\beta \beta^{*}\right)^{2} \\
& -\beta^{2} \frac{\zeta^{*}}{1-\zeta \zeta^{*}}-\beta^{* 2} \frac{\zeta}{1-\zeta \zeta^{*}} .
\end{aligned}
$$

The expectation values for the squeezed vacuum states can be taken from Appendix E where they are collected. From this follows for the expectation value of the operator $N^{2}=a^{\dagger 2} a^{2}+a^{\dagger} a$

$$
\left(\overline{N^{2}}\right)_{\beta}=\frac{\zeta \zeta^{*}\left(2+\zeta \zeta^{*}\right)}{\left(1-\zeta \zeta^{*}\right)^{2}}+\beta \beta^{*} \frac{1+3 \zeta \zeta^{*}}{1-\zeta \zeta^{*}}+\left(\beta \beta^{*}\right)^{2}-\beta^{2} \frac{\zeta^{*}}{1-\zeta \zeta^{*}}-\beta^{* 2} \frac{\zeta}{1-\zeta \zeta^{*}},
$$

and for the variance of the number operator

$$
\begin{aligned}
\left(\overline{(\Delta N)^{2}}\right)_{\beta} & =\left(\overline{N^{2}}\right)_{\beta}-(\bar{N})_{\beta}^{2} \\
& =\frac{2 \zeta \zeta^{*}}{\left(1-\zeta \zeta^{*}\right)^{2}}+\beta \beta^{*} \frac{1+\zeta \zeta^{*}}{1-\zeta \zeta^{*}}-\beta^{2} \frac{\zeta^{*}}{1-\zeta \zeta^{*}}-\beta^{* 2} \frac{\zeta}{1-\zeta \zeta^{*}} \\
& =\frac{2 \zeta \zeta^{*}}{\left(1-\zeta \zeta^{*}\right)^{2}}+\frac{\left(\beta-\zeta \beta^{*}\right)\left(\beta^{*}-\zeta^{*} \beta\right)}{1-\zeta \zeta^{*}} \geq 0 .
\end{aligned}
$$


Finally we calculate the expectation value which plays a role for the definition of sub- and super-Poissonian statistics by its sign

$$
\begin{aligned}
& \left(\overline{a^{\dagger 2} a^{2}}\right)_{\beta}-\left(\overline{a^{\dagger} a}\right)_{\beta}^{2}=\left(\overline{(\Delta N)^{2}}\right)_{\beta}-(\bar{N})_{\beta} \\
& =\frac{\zeta \zeta^{*}\left(1+\zeta \zeta^{*}\right)}{\left(1-\zeta \zeta^{*}\right)^{2}}+2 \beta \beta^{*} \frac{\zeta \zeta^{*}}{1-\zeta \zeta^{*}}-\beta^{2} \frac{\zeta^{*}}{1-\zeta \zeta^{*}}-\beta^{* 2} \frac{\zeta}{1-\zeta \zeta^{*}} \\
& =\frac{\zeta \zeta^{*}\left(1+\zeta \zeta^{*}\right)}{\left(1-\zeta \zeta^{*}\right)^{2}}-\beta \beta^{*}+\frac{\left(\beta-\zeta \beta^{*}\right)\left(\beta^{*}-\zeta^{*} \beta\right)}{1-\zeta \zeta^{*}} .
\end{aligned}
$$

The expectation values (10.6)-(10.9) depend not only on the moduli of $\beta$ and $\zeta$ but also on their phases $\mathrm{e}^{\mathrm{i} \varphi}$ and $\mathrm{e}^{\mathrm{i} \chi}$. For comparison with (10.9) one finds for the corresponding symmetrically (Weyl) ordered quantity

$$
\begin{aligned}
& \left(\overline{\mathcal{S}\left\{a^{\dagger 2} a^{2}\right\}}\right)_{\beta}-\left(\overline{\mathcal{S}\left\{a^{\dagger} a\right\}}\right)_{\beta}^{2} \\
& =\left(\overline{a^{\dagger 2} a^{2}}\right)_{\beta}-\left(\overline{a^{\dagger} a}\right)_{\beta}^{2}+\left(\overline{a^{\dagger} a}\right)_{\beta}+\frac{1}{4} \\
& =\frac{2 \zeta \zeta^{*}}{\left(1-\zeta \zeta^{*}\right)^{2}}+\frac{\left(\beta-\zeta \beta^{*}\right)\left(\beta^{*}-\zeta^{*} \beta\right)}{1-\zeta \zeta^{*}}+\frac{1}{4} \geq \frac{1}{4} .
\end{aligned}
$$

This quantity is greater than or equal to $1 / 4$ in every case but depends also on the phases of $\beta$ and $\zeta$.

\section{Sub- and Super-Poissonian Photon Statistics for Displaced Squeezed Vacuum States or Squeezed Coherent States}

The sign of the quantity (10.9) was taken by Mandel to define of sub- and super-Poissonian statistics as follows [11]

$$
\begin{aligned}
& \overline{a^{\dagger 2} a^{2}}-\left(\overline{a^{\dagger} a}\right)^{2}=\overline{(\Delta N)^{2}}-\bar{N}<0, \quad \text { (sub-Poissonian), } \\
& \overline{a^{\dagger 2} a^{2}}-\left(\overline{a^{\dagger} a}\right)^{2}=\overline{(\Delta N)^{2}}-\bar{N}>0, \quad \text { (super-Poissonian). }
\end{aligned}
$$

We now investigate the photon statistics of this quantity for displaced squeezed vacuum states starting from (10.9). First we denote the phases of $\beta$ and $\zeta$ in the the complex plane as follows

$$
\beta=|\beta| \mathrm{e}^{\mathrm{i} \varphi}, \quad \zeta=|\zeta| \mathrm{e}^{\mathrm{i} \chi} .
$$

The mean value $\bar{N}$ of the number operator $N$ according to (10.5) does not depend on the angle between $\beta$ and $\zeta$ and if we change only the angle $\varphi$ of $\beta$ leaving $|\beta|$ and $\zeta$ constant the mean value $\bar{N}$ remains constant and the squeezing ellipses change in their position in comparison to the displacement $\beta$. This is shown in Figure 2. In the following we show that the photon statistics may change from sub- to super-Poissonian statistics if the displacement $\beta$ moves around the circles shown schematically in Figure 2 and 
if $|\beta|$ is sufficiently large and somewhere between this has to be a position where it possesses the same values of $\bar{N}=\overline{a^{\dagger} a}$ and $\overline{a^{\dagger 2} a^{2}}-\left(\overline{a^{\dagger} a}\right)^{2}$ as the Poisson statistics of coherent states with the same $\bar{N}$.

From (10.9) and from definition (11.1) follows as condition for subPoissonian statistics of squeezed coherent states $|\beta, \zeta\rangle$

$$
\frac{|\zeta|^{2}\left(1+|\zeta|^{2}\right)}{\left(1-|\zeta|^{2}\right)^{2}}-2|\beta|^{2} \frac{|\zeta|\left(\cos \left(\chi_{0}\right)-|\zeta|\right)}{1-|\zeta|^{2}}<0, \quad\left(\chi_{0} \equiv \chi-2 \varphi\right)
$$

or resolved to $|\beta|^{2}$ for $\cos \left(\chi_{0}\right)-|\zeta|>0$ (pay attention that in case of $\cos \left(\chi_{0}\right)-|\zeta|<0$ the inequality (11.3) cannot be satisfied or changes "> into " $<$ " if divided by $\cos \left(\chi_{0}\right)-|\zeta|<0$ !)

$$
|\beta|^{2}>\frac{|\zeta|\left(1+|\zeta|^{2}\right)}{2\left(1-|\zeta|^{2}\right)\left(\cos \left(\chi_{0}\right)-|\zeta|\right)}>0, \quad\left(\cos \left(\chi_{0}\right)>|\zeta|\right) .
$$

For possible sub-Poissonian statistics it is necessary that $\cos \left(\chi_{0}\right)>|\zeta|$ whereas in case of $\cos \left(\chi_{0}\right)<|\zeta|$ we have super-Poissonian statistics. For $\chi_{0}=0$ or $\cos \left(\chi_{0}\right)-|\zeta|=1-|\zeta|>0$ that means for squeezing in direction of the displacement parameter $\beta$ (amplitude squeezing, see Figure 3 ) the necessary condition for sub-Poissonian statistics is satisfied but according to (11.4) the modulus $|\beta|$ of the displacement parameter has to be greater than a minimal value defined by the equality sign in (11.4). For $\chi_{0}=\pi$ or $\cos \left(\chi_{0}\right)-|\zeta|=-1-|\zeta|<0$ the left-hand side of (11.3) is positive and we have super-Poissonian statistics. Thus one of the necessary conditions for sub-Poissonian statistics is (see Figure 4, inner circle)

$$
\cos \left(\chi_{0}\right)-|\zeta|>0
$$

with the limiting curve by substitution of " $>$ " by the equality sign "=" in (11.5). In the other necessary condition (11.4) it is better to substitute the displacement parameter $|\beta|$ by the expectation value $\bar{N}$ of the number operator since it is then easier to compare the results with coherent states with the same $\bar{N}$.

We express now the displacement parameter $\beta$ in (10.9) by the expectation value $\bar{N}$ of the number operator $N$ using (10.5)

$$
|\beta|^{2}=\bar{N}-\frac{|\zeta|^{2}}{1-|\zeta|^{2}} \geq 0
$$

where we have omitted the index $\beta$ in $(\bar{N})_{\beta}$ since we use it in the following also for coherent states. If we do so then we obtain from (10.9) with abbreviation $\chi_{0} \equiv \chi-2 \varphi$

$$
\begin{aligned}
& \left(\overline{a^{\dagger 2} a^{2}}\right)_{\beta}-\left(\overline{a^{\dagger} a}\right)_{\beta}^{2}=\frac{|\zeta|^{2}\left(1+|\zeta|^{2}\right)}{\left(1+|\zeta|^{2}\right)^{2}}-2\left(\bar{N}-\frac{|\zeta|^{2}}{1-|\zeta|^{2}}\right) \frac{|\zeta|\left(\cos \left(\chi_{0}\right)-|\zeta|\right)}{1-|\zeta|^{2}} \\
& =\frac{|\zeta|}{\left(1-|\zeta|^{2}\right)^{2}}\left\{|\zeta|\left(1+|\zeta|^{2}\right)-2\left(\bar{N}-(\bar{N}+1)|\zeta|^{2}\right)\left(\cos \left(\chi_{0}\right)-|\zeta|\right)\right\} .
\end{aligned}
$$




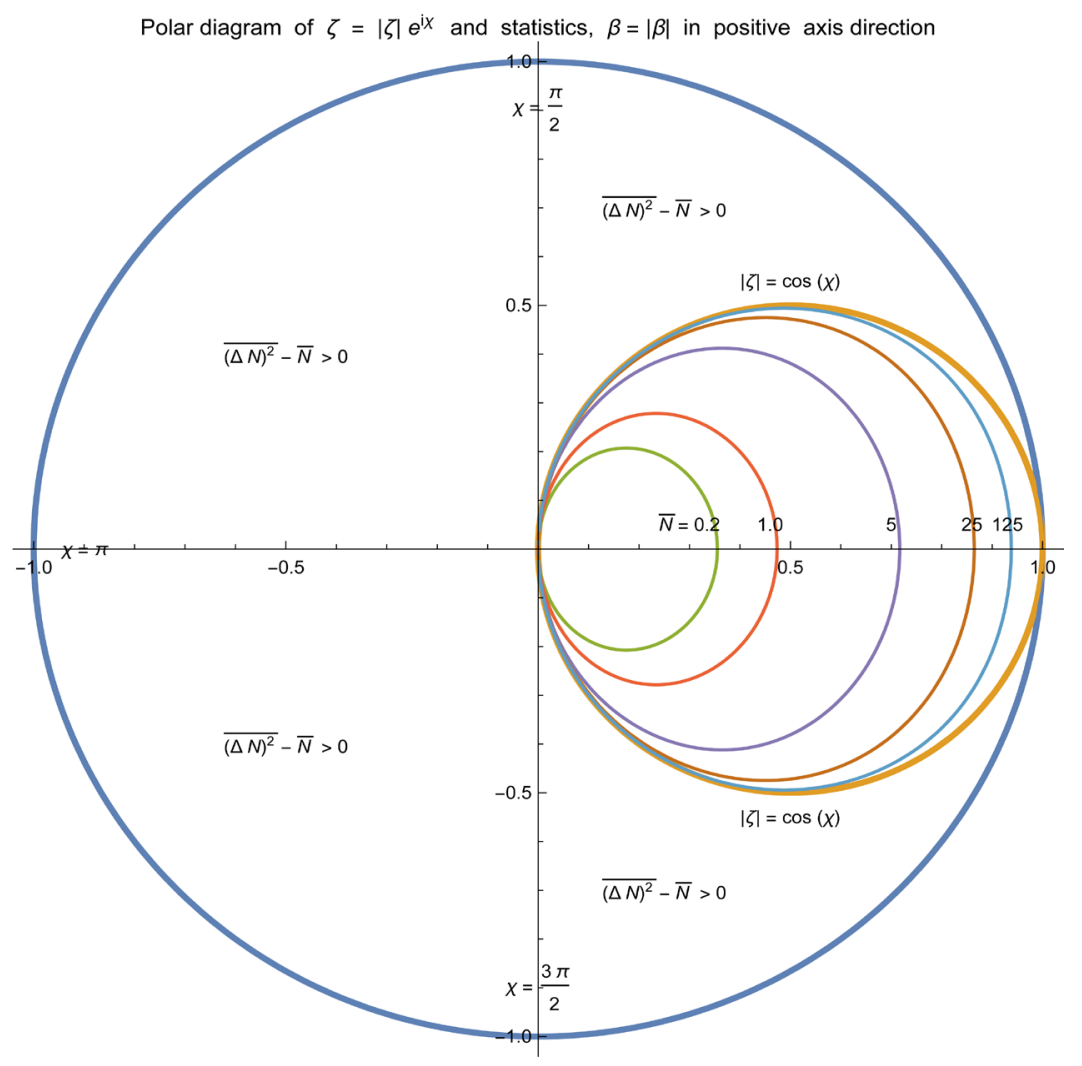

Figure 4. Polar diagram $|\zeta|=f(\chi)$ of sub- and super-Poissonian statistics in dependence on the complex squeezing parameter in non-unitary approach $\zeta=|\zeta| \mathrm{e}^{\mathrm{i} x}$ and with displacement parameter $\beta=|\beta| \mathrm{e}^{\mathrm{i} \varphi}$ with fixed $\varphi=0$ (in positive axis direction) and therefore $\chi_{0} \equiv \chi-2 \varphi=\chi$. Sub-Poissonian statistics is only possible within the inner circle $|\zeta|=\cos \left(\chi_{0}\right)$. Within this circle the ellipse-like curves with constant $\bar{N}$ separate regions of sub-Poissonian statistics from regions of super-Poissonian statistics where sub-Poissonian statistics is within the ellipse-like inner regions to the crescent-like outer regions with super-Poissonian statistics. The center $\zeta=0 \quad(\chi$ arbitrary) corresponds to coherent states of arbitrary $\bar{N}$. Constant $|\zeta|$ (circles around coordinate origin) correspond for fixed $\beta=|\beta|$ to constant $\bar{N}$ touching the separatrices with the same $\bar{N}$ at $\chi=0$. Using the squeezing parameter $\zeta^{\prime}$ in the unitary approach (see (5) and (6)) this scheme would take on the whole complex plane and, in particular, for parameters $\zeta$ with $|\zeta| \rightarrow 1$ one has a large stretching of corresponding parameters $\zeta^{\prime}$ with $\left|\zeta^{\prime}\right| \rightarrow \infty$ and the inner circle $|\zeta|=\cos (\chi)$ becomes $\left|\zeta^{\prime}\right|=\operatorname{Arth}(\cos (\chi))$.

Setting this expression equal to zero one obtains an equation for states which belong neither to sub- nor to super-Poissonian statistics. This is a third-order equation for $|\zeta|$ in dependence on $\bar{N}$ and on the angle $\chi_{0}$ as follows

$$
|\zeta|^{3}-\frac{2(\bar{N}+1) \cos \left(\chi_{0}\right)}{2 \bar{N}+1}|\zeta|^{2}-|\zeta|+\frac{2 \bar{N} \cos \left(\chi_{0}\right)}{2 \bar{N}+1}=0 .
$$

As a third-order equation for $|\zeta|$ with real coefficients in dependence on $\bar{N}$ and $\chi_{0}$ it may possess, in principal, three real or one real and two complex 
conjugate solutions but for our purpose the real solutions have to be positive ones and have to be restricted to $|\zeta|<1$. The results are presented in Figure 4 as polar diagram of $\zeta=|\zeta| \mathrm{e}^{\mathrm{i} x}$. The ellipse-like regions of the inner smaller circle (11.5) in Figure 4 belong to sub-Poissonian and the outer crescent-like regions to super-Poissonian statistics in dependence on $\bar{N}$ shown as separatrices.

Figure 5 shows that between sub- and super-Poissonian statistics may lie statistics which are very far from a Poisson statistics, in case of $|\zeta| \rightarrow 1$ even maximally far (distance $d$ to nearest coherent states goes to $\sqrt{2}$ ). Alternatively to Figure 5 one may demonstrate the existence of displaced squeezed vacuum states with $\overline{(\Delta N)^{2}}-\bar{N}=0$ also in the following way. One begins with an arbitrarily chosen displacement parameter $\zeta$ and looks according to condition (11.4) for displacement parameters $\beta$ above the minimal possible one for

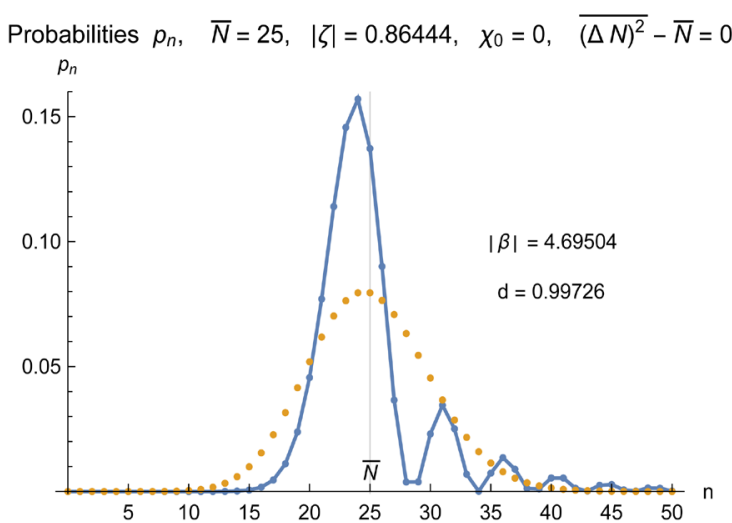

Probabilities $p_{n}, \quad \bar{N}=25, \quad|\zeta|=0.67058, \quad \chi_{0}= \pm \frac{\pi}{4}, \quad \overline{(\Delta N)^{2}}-\bar{N}=0$

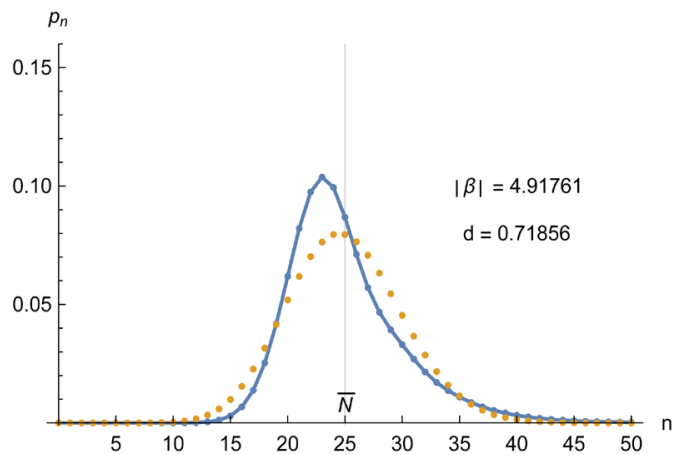

Probabilities $p_{n}, \quad \bar{N}=25, \quad|\zeta|=0.82723, \quad \chi_{0}= \pm \frac{\pi}{8}, \quad \overline{(\Delta N)^{2}}-\bar{N}=0$

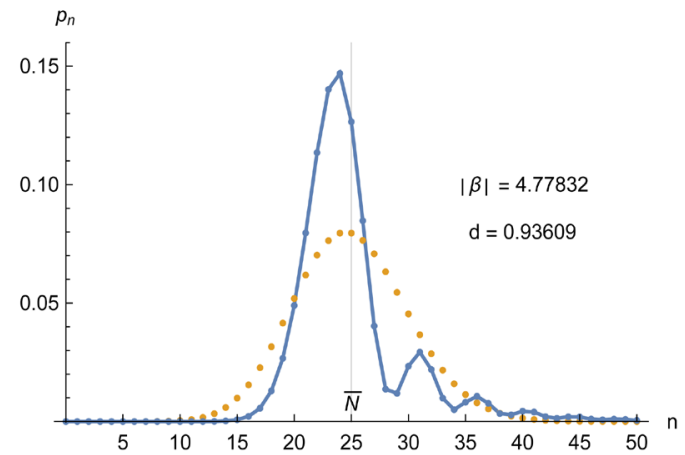

Probabilities $p_{n}, \quad \bar{N}=25, \quad|\zeta|=0.37276, \quad \chi_{0}= \pm \frac{3 \pi}{8}, \quad \overline{(\Delta N)^{2}}-\bar{N}=0$

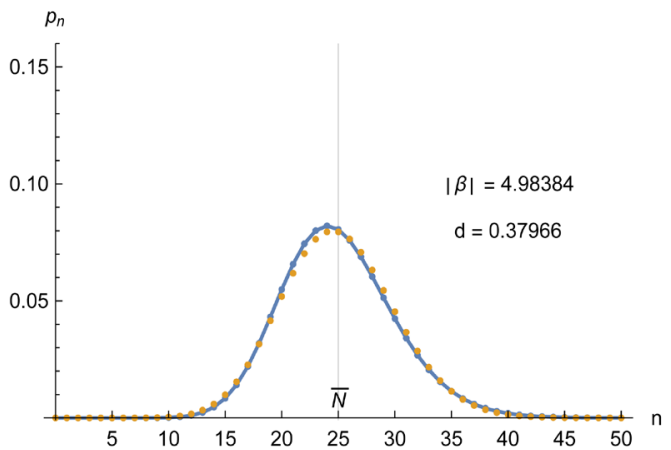

Figure 5. Probabilities $p_{n}$ for squeezed coherent states (joined points) with $\bar{N}=25$ and $\overline{(\Delta N)^{2}}-\bar{N}=0$ such as for a coherent state (orange points) with $\bar{N}=25$ and nearest distance $d$ to coherent states $|\beta\rangle$ with $\bar{N}=\beta \beta^{*}$ or $\bar{a}=\beta$. Additionally, the displacement parameter $|\beta|$ is given. The last partial figure with small squeezing parameter $|\zeta|$ is in its photon statistics already "visibly" near to a coherent state with $\bar{N}=25$ but the nearest distance to a coherent state (with $\bar{N}=\beta \beta^{*}=24.8387$ ) is not yet very small and shows that this measure is very sensible. All four partial figures belong to states with photon statistics which is neither sub- nor super-Poissonian but also not a Poisson statistics. For $|\zeta|=0, \zeta=0, \chi=\chi_{0}= \pm \frac{\pi}{2}$ (but, in principle, arbitrary) one has exactly a coherent state (see also Figure 4, center of diagram). 
which one has sub-Poissonian statistics if $\beta$ possesses the same or the opposite direction of $\zeta$ (amplitude squeezing). Then by rotating the phase $\mathrm{e}^{\mathrm{i} \chi}$ of $\zeta$ from $\chi=0$ (amplitude squeezing) up to $\chi=\pi$ (phase squeezing) leaving constant the modulus $|\zeta|$ (see Figure 1, right partial figure) the photon statistics makes the transition from sub- to super-Poissonian statistics and one comes unavoidable to a value of the phase $\chi$ where $\overline{(\Delta N)^{2}}-\bar{N}=0$ but which does not belong to Poisson statistics. The same effect one has if we rotate the displacement parameter $\beta$ leaving constant the squeezing parameter $\zeta$ in modulus and phase (see Figure 3). If the time evolution of a squeezed state is determined by a Hamiltonian $H$ which is a linear combination of $a^{2}, a^{2}$ and

of $\frac{1}{2}\left(a a^{\dagger}+a^{\dagger} a\right)$ then there may appear a complicated picture of changing with time from sub- to super-Poissonian statistics or from amplitude to phase squeezing since then also the modulus of the squeezing parameter changes with time.

Clearly, one may make the division of photon statistics in sub- and superPoissonian ones but both categories are very inhomogeneous concerning the comprised states and the set of states which are neither sub- nor superPoissonian ones is also very large and inhomogeneous and the prefixes "sub" and "super" are here problematic. There are hardly to expect clear differences and correlations in experiments with states of both statistics or, moreover, even qualitatively different behavior. In general, a photon statistics is determined by a countable infinite number of parameters (e.g., $p_{n}$ or moments of the distribution) and for states which belong neither to sub- nor to super-Poissonian statistics only one from this countable infinite set is fixed $\left((\Delta N)^{2}-\bar{N}=0\right)$ and this can be considered in dependence on arbitrary $\bar{N}$. Therefore, also the classification of states with sub-Poissonian photon statistics as nonclassical states is highly problematic. In the same way it is also problematic to define the states with no regions of negativity of the Wigner quasiprobability as the classical states since then the set of these states is too large and inhomogeneous to be useful for comparative purpose (all squeezed coherent states belong then to them). Better seems to be for this purpose to use the nearest distance to a coherent state as quantitative measure but this measure cannot change its sign and is in every case positive or zero [20] [21]. In this definition it is evident that the category of states with large distance to the nearest coherent states is very inhomogeneous and large.

\section{Conclusions}

A main purpose of this article was to discuss the distinction of cases of sub- and super-Poissonian statistics within the displaced squeezed vacuum states where the non-unitary approach is preferable. For this case it was necessary to calculate the expectation values of powers of the number operator for these states. We have chosen for this purpose mainly its calculation from the number-state 
representation and posed this in a more general connection to the calculation of properties of these states. For squeezed coherent states, practically, all interesting parameters can be calculated in exact and not very difficult way and, therefore, this category of states is very suited to demonstrate in examples more principal definitions for all states.

The means developed in this article can be applied without substantial changes to squeezing of the number state $|1\rangle$ and then to its displacement since the operator $\exp \left(-\frac{\zeta^{*}}{2} a^{2}\right)$ in its disentanglement (2.7)) acts on $|1\rangle$ by reproducing it (case $k=\frac{3}{4}$ in (A.4)). This becomes more complicated if we apply it to the number states $|n\rangle$ with $n \geq 2$ and to extend the theory to these cases.

\section{References}

[1] Schrödinger, E. (1926) Der stetige Übergang von der Mikro zur Makromechanik. Naturwiss. 14, 664-666. https://doi.org/10.1007/BF01507634

[2] Pauli, W. (1958) Die allgemeinen Prinzipien der Wellenmechanik. Handbuch der Physik, Band V, Teil 1, Herausgegeben von S. Flügge. Springer, Berlin, 1-168. (This Is a Republication of the Same Article with Small Changes in Old "Handbuch der Physik", Band XXIV, Teil 1, herausgegeben von Geiger und Scheel, 1933). https://doi.org/10.1007/978-3-642-80539-4_1

[3] Louisell, W.H. (1973) Quantum Statistical Properties of Radiation. John Wiley \& Sons, New York.

[4] Walls, D.F. (1983) Squeezed States of Light. Nature, 306, 141. https://doi.org/10.1038/306141a0

[5] Kimble, J. and Walls, D.F. (1987) Squeezed States of the Electromagnetic Field. Journal of the Optical Society of America (JOSA), B4, 10.

[6] Loudon, R. and Knight, P.L. (1987) Squeezed Light. Journal of Modern Optics, 34, 709. https://doi.org/10.1080/09500348714550721

[7] Perelomov, A.M. (1977) Generalized Coherent States and Some of Their Applications. Uspekhi Fizicheskih Nauk, 123, 23-55 (1977) (In Russian). Soviet Physics Uspekhi, 20, 703 (In English). https://doi.org/10.3367/UFNr.0123.197709b.0023

[8] Perelomov, A. (1986) Generalized Coherent States and Their Application. Springer, Berlin. https://doi.org/10.1007/978-3-642-61629-7

[9] Perelomov, A.M. (1987) Obobstschonnyje kogerentnyje sostoyanya i ikh primenyenyje. Nauka, Moskva (Is Not Fully Identical with [8]).

[10] Walls, D.F. and Milburn, G.J. (1994) Quantum Optics. Springer, Berlin. https://doi.org/10.1007/978-3-642-79504-6

[11] Mandel, L. and Wolf, E. (1995) Optical Coherence and Quantum Optics. Cambridge University Press, Cambridge. https://doi.org/10.1017/CBO9781139644105

[12] Scully, M.O. and Zubairy, M.S. (1997) Quantum Optics. Cambridge University Press, Cambridge. https://doi.org/10.1017/CBO9780511813993

[13] Schleich, W. (2001) Quantum Optics in Phase Space. Wiley-VCH, Berlin. https://doi.org/10.1002/3527602976

[14] Leonhardt, U. (1997) Measuring the Quantum State of Light. Cambridge University 
Press, Cambridge.

[15] Fan, H.-Y. (1990) Squeezed States for Two Types of One- and Two-Mode Squeezing Transformations. Physical Review A, 41, 1526-1532. https://doi.org/10.1103/PhysRevA.41.1526

[16] Bužek, V. (1989) Time Evolution of an Anharmonic Oscillator in an Initial Holstein-Primakoff SU(1,1) Coherent State. Physical Review A, 39, 5432. https://doi.org/10.1103/PhysRevA.39.5432

[17] Vourdas, A. (1993) Phase States: An Analytic Approach in the Unit Disc. Physica Scripta T, 48, 84. https://doi.org/10.1088/0031-8949/1993/T48/012

[18] Wünsche, A. (2003) Squeezed States. In: Dodonov, V.V., Man'ko, V.I., Taylor and Francis, Eds., Theory of Nonclassical States of Light, London and New York, 95-152.

[19] Dodonov, V.V. (2002) Nonclassical States in Quantum Optics: A “Squeezed” Review of First 75 Years. Journal of Optics B: Quantum and Semiclassical Optics, 4, No. 1. https://doi.org/10.1088/1464-4266/4/1/201

[20] Wünsche, A. (1995) The Distance to Poissonian Statistics as a Supplementary Measure in Quantum Optics. Journal of Applied Physics, 60, 119-122.

[21] Dodonov, V.V., Man'ko, O.V., Man'ko, V.I. and Wünsche, A. (2000) Hilbert-Schmidt Distance and Nonclassicality of States in Quantum Optics. Journal of Modern Optics, 47, 633-654. https://doi.org/10.1080/09500340008233385

[22] Wünsche, A. (2017) About Classical to Quantum Weyl Correspondence. Applied Mathematics, 07, No. 10. https://doi.org/10.4236/apm.2017.710034

[23] Dodonov, V.V., Man'ko, O.V. and Man'ko, V.I. (1994) Photon Distribution for One-Mode Mixed Light with a Generic Gaussian Wigner Function. Physical Review $A$, 49, 2993. https://doi.org/10.1103/PhysRevA.49.2993

[24] Wünsche, A. (2015) Quantum-Mechanical Cumulant Expansions and Their Application to Phase-Space and to Phase Distributions. Physica Scripta, 90, Article ID: 074063. https://doi.org/10.1088/0031-8949/90/7/074063

[25] Klauder, J.R. and Sudarshan, E.C.G. (1968) Fundamentals of Quantum Optics. W.A. Benjamin, Inc., New York.

[26] Klauder, J.R. and Skagerstam, B.-S. (1985) Coherent States, Chapter I: A Coherent-State Primer. World Scientific, Singapore.

[27] Wigner, E. (1932) On the Quantum Correction For Thermodynamic Equilibrium. Physical Review, 40, 749 (Republished in [28]). https://doi.org/10.1103/PhysRev.40.749

[28] Kim, Y.S. and Noz, M.E. (1991) Phase Space Picture of Quantum Mechanics. World Scientific, Singapore (With Republication of Article [27]).

https://doi.org/10.1142/1197

[29] Wünsche, A. (1996) The Complete Gaussian Class of Quasiprobabilities and Its Relation to Squeezed States and Their Excitations, Quantum Semiclass. Optical, 8, 343-379.

[30] Szegö, G. (1959) Orthogonal Polynomials. 2nd Edition, American Mathematical Society, New York (1st Edition 1939).

[31] Bateman, H. and Erdélyi, A. (1953) Higher Transcendental Functions, Vol. II. McGraw-Hill, New York.

[32] Wünsche, A. (2015) Operator Methods and SU(1,1) Symmetry in the Theory of Jacobi and of Ultraspherical Polynomials. Applied Mathematics, 7, 213-261. 
[33] Akhiezer, N.I. and Glazman, I.M. (2013) Teoriya lineinykh operatorov v gilbertovym prostranstve, Nauka, Moskva 1966. English Translation: Theory of Linear Operators in Hilbert Space, Dover, New York.

[34] Vourdas, A. and Wünsche, A. (1998) Resolutions of the Identity in Terms of Line Integrals of $S U(1,1)$ Coherent States. Journal of Physics A: Mathematical and General, 31, 9341-9352.

https://doi.org/10.1088/0305-4470/31/46/024

[35] Wünsche, A. (1997) Radon Transform and Pattern Functions in Quantum Tomography. Journal of Modern Optics, 44, 2293-2331.

https://doi.org/10.1080/09500349708231885 


\section{Appendix A}

\section{Squeezing Operators and Their Disentanglement}

Squeezing of states in the narrow sense is connected with the Lie group $S U(1,1) \cong S p(2, \mathbb{R}) \cong S L(2, \mathbb{R})$ with 3 real parameters (or one complex and one real in our representation) of squeezing operators [7] [8] [9]. Its complex extension $S p(2, \mathbb{C}) \cong S L(2, \mathbb{C})$ contains 3 complex parameters (or 6 real ones) and comprises also the $S U(2)$ group in the right specialization. In this Appendix we give a short but general and systematic representation of the action of $S U(1,1)$ squeezing operators in quantum optics. We cannot take care without making too clumsy notations in some cases to the notations in the main text but this concerns mostly the notations of the squeezing parameters with and without prime (or in non-unitary and unitary approaches) and one must pay attention to this when going from formulae of this Appendix to that of the main text.

We define operators $S(\xi, \eta, \zeta)$ with 3 complex parameters which are operators in $S L(2, \mathbb{C})$ as follows

$$
S(\xi, \eta, \zeta) \equiv \exp \left\{\xi K_{-}+\mathrm{i} 2 \eta K_{0}-\zeta K_{+}\right\}
$$

where $\left(K_{-}, K_{0}, K_{+}\right)$are three abstract operators of the Lie algebra $\operatorname{sl}(2, \mathbb{C})$ to the Lie group $S L(2, \mathbb{C})$ satisfying the commutation relation [7] [8] [9] [34]

$$
\left[K_{-}, K_{+}\right]=2 K_{0},\left[K_{0}, K_{-}\right]=-K_{-},\left[K_{0}, K_{+}\right]=+K_{+},
$$

with Casimir operator $C$

$$
C \equiv K_{0}^{2}-K_{1}^{2}-K_{2}^{2}=K_{0}^{2}-\frac{1}{2}\left(K_{-} K_{+}+K_{+} K_{-}\right), \quad\left[C, K_{i}\right]=0 .
$$

One basic discrete realization of these operators in quantum optics of a single mode is

$$
\begin{gathered}
K_{-} \equiv \frac{1}{2} a^{2}=\frac{1}{4 \hbar}\left(Q^{2}-P^{2}+\mathrm{i}(Q P+P Q)\right), \\
K_{0} \equiv \frac{1}{4}\left(a a^{\dagger}+a^{\dagger} a\right)=\frac{1}{4 \hbar}\left(Q^{2}+P^{2}\right), \\
K_{+} \equiv \frac{1}{2} a^{\dagger 2}=\frac{1}{4 \hbar}\left(Q^{2}-P^{2}-\mathrm{i}(Q P+P Q)\right), \\
C \equiv k(k-1) I=-\frac{3}{16}, \quad k=\frac{1}{4}, \quad\left(\text { or } k=\frac{3}{4}\right),
\end{gathered}
$$

where $\left(a, a^{\dagger}\right)$ is a pair of boson annihilation and creation operators (A.1) and $k=\frac{3}{4}$ belongs to squeezed number states $|1\rangle$ (and their displacement) not dealt with in this article. Each realization of the commutation relations (A.2) is appropriate for the following derivations but for the two-dimensional fundamental representation it is important to know two basic operators for which $\left(a, a^{\dagger}\right)$ are particularly convenient in combination with the realization (A.4). Squeezing with $S U(1,1)$ operators within two modes and genuinely 
different from single-mode squeezing is also possible [8] [15]. A further realization of $S U(1,1)$ for a single mode is connected with coherent phase states.

The transition from $S L(2, \mathbb{C})$ to $S L(2, \mathbb{R}) \cong S U(1,1)$ can be made by specializing the parameters $(\xi, \eta, \zeta)$ in (A.1) according to

$$
\begin{aligned}
S\left(\zeta^{*}, \eta=\eta^{*}, \zeta\right) & =\exp \left\{\frac{\zeta^{*}}{2} a^{2}+\mathrm{i} \frac{\eta}{2}\left(a a^{\dagger}+a^{\dagger} a\right)-\frac{\zeta}{2} a^{\dagger 2}\right\} \\
& =\left(S\left(-\zeta^{*},-\eta=-\eta^{*},-\zeta\right)\right)^{\dagger}
\end{aligned}
$$

with complex parameter $\zeta$ and real parameter $\eta$. The operators $S\left(\zeta^{*}, \eta=\eta^{*}, \zeta\right)$ are then unitary operators $S^{-1}=S^{\dagger}$ in the infinite-dimensional unitary representation in Hilbert space and we have $\left(K_{-}^{\dagger}, K_{0}^{\dagger}, K_{+}^{\dagger}\right)=\left(K_{+}, K_{0}, K_{-}\right)$. Instead of $\left(K_{-}, K_{+}\right)$one may introduce Hermitean operators $\left(K_{1}, K_{2}\right)$ by $K_{\mp} \equiv K_{1} \mp \mathrm{i} K_{2}$ but we do not write down all relations for these new operators that is easy to make and is diligent work.

We now consider the generation of the two-dimensional fundamental representation of the group $S L(2, \mathbb{C})$ by calculating the matrix with elements $(\kappa, \lambda, \mu, v)$ in the following relation

$$
S(\xi, \eta, \zeta)\left(a, a^{\dagger}\right)(S(\xi, \eta, \zeta))^{-1}=\left(a, a^{\dagger}\right)\left(\begin{array}{ll}
\kappa & \lambda \\
\mu & v
\end{array}\right)=\left(\kappa a+\mu a^{\dagger}, \lambda a+v a^{\dagger}\right) .
$$

This step is essentially a Bogolyubov transformation. It can be represented by (e.g., [18])

$$
\begin{aligned}
\left(\begin{array}{ll}
\kappa & \lambda \\
\mu & \nu
\end{array}\right) & =\left(\begin{array}{cc}
\operatorname{ch}(\varepsilon)-\mathrm{i} \eta \frac{\operatorname{sh}(\varepsilon)}{\varepsilon} & \xi \frac{\operatorname{sh}(\varepsilon)}{\varepsilon} \\
\zeta \frac{\operatorname{sh}(\varepsilon)}{\varepsilon} & \operatorname{ch}(\varepsilon)+\mathrm{i} \eta \frac{\operatorname{sh}(\varepsilon)}{\varepsilon}
\end{array}\right) . \\
\varepsilon & \equiv \sqrt{\xi \zeta-\eta^{2}} .
\end{aligned}
$$

For $\varepsilon^{2}>0,(\varepsilon$ real) we call this transformation squeezing-like and for $\varepsilon^{2}<0$, ( $\varepsilon$ imaginary) rotation-like. In last case it is better to write the hyperbolic functions by trigonometric functions. The special case $\varepsilon=0$

$$
\left(\begin{array}{ll}
\kappa & \lambda \\
\mu & v
\end{array}\right)=\left(\begin{array}{cc}
1-\mathrm{i} \eta, & \xi \\
\zeta, & 1+\mathrm{i} \eta
\end{array}\right), \quad \xi \zeta=\eta^{2}
$$

could be called cone-like. The matrices (A.7) are unimodular

$$
\left|\left(\begin{array}{ll}
\kappa & \lambda \\
\mu & v
\end{array}\right)\right|=\kappa v-\lambda \mu=1
$$

but in general, not unitary.

The inversion of (A.7) which is unique can be simply written

$$
\xi=\lambda \frac{\operatorname{Arsh}(\vartheta)}{\vartheta}, \quad \eta=\mathrm{i} \frac{\kappa-\nu}{2} \frac{\operatorname{Arsh}(\vartheta)}{\vartheta}, \quad \zeta=\mu \frac{\operatorname{Arsh}(\vartheta)}{\vartheta},
$$

with the following relations between the abbreviations $\varepsilon$ and $\vartheta$ 


$$
\begin{gathered}
\varepsilon=\operatorname{Arch}\left(\frac{\kappa+v}{2}\right)=\operatorname{Arsh}\left(\sqrt{\left(\frac{\kappa+v}{2}\right)^{2}-1}\right)=\operatorname{Arsh}\left(\sqrt{\left(\frac{\kappa-v}{2}\right)^{2}+\lambda \mu}\right) \equiv \operatorname{Arsh}(\vartheta) \\
\vartheta \equiv \operatorname{sh}(\varepsilon)=\sqrt{\left(\frac{\kappa+v}{2}\right)^{2}-1}=\sqrt{\left(\frac{\kappa-v}{2}\right)^{2}+\lambda \mu}
\end{gathered}
$$

From $\operatorname{Arch}(z)$ the sign of $\operatorname{Arsh}\left(\sqrt{z^{2}-1}\right)$ does not follow uniquely but $\frac{\operatorname{Arsh}\left(\sqrt{z^{2}-1}\right)}{\sqrt{z^{2}-1}}$ is then uniquely determined if one chooses the same sign of $\sqrt{z^{2}-1}$ in numerator and denominator.

We now write down some special correspondences between the operators $S(\xi, \eta, \zeta)$ and its two-dimensional matrices in the fundamental representation

$$
\begin{gathered}
S(\xi, 0,0)=\exp \left(\xi K_{-}\right) \rightarrow \exp \left(\frac{\xi}{2} a^{2}\right), \Leftrightarrow\left(\begin{array}{cc}
1, & \xi \\
0, & 1
\end{array}\right), \\
S(0, \eta, 0)=\exp \left(\mathrm{i} \eta 2 K_{0}\right) \rightarrow \exp \left\{\mathrm{i} \frac{\eta}{2}\left(a a^{\dagger}+a^{\dagger} a\right)\right\} \Leftrightarrow\left(\begin{array}{cc}
\mathrm{e}^{-\mathrm{i} \eta}, & 0 \\
0, & \mathrm{e}^{\mathrm{i} \eta}
\end{array}\right), \\
S(0,0, \zeta)=\exp \left(-\zeta K_{+}\right) \rightarrow \exp \left(-\frac{\zeta}{2} a^{\dagger 2}\right), \Leftrightarrow\left(\begin{array}{cc}
1, & 0 \\
\zeta, & 1
\end{array}\right) .
\end{gathered}
$$

The triangular operators $S(\xi, 0,0)$ and $S(0,0, \zeta)$ and their correspondent two-dimensional matrices form a group for themselves and in the same way the operators $S(0, \eta, 0)$ together with its matrices. However, the operators $S(\xi, 0, \zeta)$ do not form a group its extension to a group needs all operators $S(\xi, \eta, \zeta)$.

We may decompose the matrices in (A.7) into products of special matrices, in particular, in the following for us important ways

$$
\begin{aligned}
\left(\begin{array}{ll}
\kappa & \lambda \\
\mu & v
\end{array}\right) & =\left(\begin{array}{cc}
1 & 0 \\
\frac{\mu}{\kappa} & 1
\end{array}\right)\left(\begin{array}{cc}
1 & \lambda \kappa \\
0 & 1
\end{array}\right)\left(\begin{array}{ll}
\kappa & 0 \\
0 & \frac{1}{\kappa}
\end{array}\right) \\
& =\left(\begin{array}{ll}
1 & 0 \\
\frac{\mu}{\kappa} & 1
\end{array}\right)\left(\begin{array}{ll}
\kappa & 0 \\
0 & \frac{1}{\kappa}
\end{array}\right)\left(\begin{array}{cc}
1 & \frac{\lambda}{\kappa} \\
0 & 1
\end{array}\right) \\
& =\left(\begin{array}{ll}
\kappa & 0 \\
0 & \frac{1}{\kappa}
\end{array}\right)\left(\begin{array}{cc}
1 & 0 \\
\mu \kappa & 1
\end{array}\right)\left(\begin{array}{cc}
1 & \frac{\lambda}{\kappa} \\
0 & 1
\end{array}\right) \\
& =\left(\begin{array}{ll}
\frac{1}{v} & 0 \\
0 & v
\end{array}\right)\left(\begin{array}{cc}
1 & \lambda v \\
0 & 1
\end{array}\right)\left(\begin{array}{cc}
1 & 0 \\
\frac{\mu}{v} & 1
\end{array}\right) \\
& =\left(\begin{array}{ll}
1 & \frac{\lambda}{v} \\
0 & 1
\end{array}\right)\left(\begin{array}{cc}
\frac{1}{v} & 0 \\
0 & v
\end{array}\right)\left(\begin{array}{cc}
1 & 0 \\
\frac{\mu}{v} & 1
\end{array}\right) \\
& =\left(\begin{array}{ll}
1 & \frac{\lambda}{v} \\
0 & 1
\end{array}\right)\left(\begin{array}{cc}
1 & 0 \\
\mu \nu & 1
\end{array}\right)\left(\begin{array}{ll}
\frac{1}{v} & 0 \\
0 & v
\end{array}\right), \quad \kappa v-\lambda \mu=1 .
\end{aligned}
$$


As consequences we obtain the following disentanglements of the general operator $S(\xi, \eta, \zeta)$ into products of partial operators

$$
\begin{aligned}
S(\xi, \eta, \zeta) & \equiv \exp \left(\xi K_{-}+\mathrm{i} 2 \eta K_{0}-\zeta K_{+}\right) \\
& =\exp \left(-\frac{\mu}{\kappa} K_{+}\right) \exp \left(\lambda \kappa K_{-}\right) \exp \left(-2 \log (\kappa) K_{0}\right) \\
& =\exp \left(-\frac{\mu}{\kappa} K_{+}\right) \exp \left(-2 \log (\kappa) K_{0}\right) \exp \left(\frac{\lambda}{\kappa} K_{-}\right) \\
& =\exp \left(-2 \log (\kappa) K_{0}\right) \exp \left(-\mu \kappa K_{+}\right) \exp \left(\frac{\lambda}{\kappa} K_{-}\right) \\
& =\exp \left(2 \log (v) K_{0}\right) \exp \left(\lambda v K_{-}\right) \exp \left(-\frac{\mu}{v} K_{+}\right) \\
& =\exp \left(\frac{\lambda}{v} K_{-}\right) \exp \left(2 \log (v) K_{0}\right) \exp \left(-\frac{\mu}{v} K_{+}\right) \\
& =\exp \left(\frac{\lambda}{v} K_{-}\right) \exp \left(-\mu \nu K_{+}\right) \exp \left(2 \log (v) K_{0}\right) .
\end{aligned}
$$

For the operators $\left(K_{-}, K_{0}, K_{+}\right)$we may insert the realization (A.4) of $S U(1,1)$ but every other realization of $S L(2, \mathbb{C})$ is also appropriate.

In the special case $\eta=0$ of operators $S(\xi, \eta, \zeta)$ the matrices (A.7) specialize

$$
S(\xi, 0, \zeta) \Leftrightarrow\left(\begin{array}{ll}
\kappa & \lambda \\
\mu & \nu
\end{array}\right)=\left(\begin{array}{cc}
\operatorname{ch}(\sqrt{\xi \zeta}) & \xi \frac{\operatorname{sh}(\sqrt{\xi \zeta})}{\sqrt{\xi \zeta}} \\
\operatorname{sh}(\sqrt{\xi \zeta}) & \operatorname{ch}(\sqrt{\xi \zeta})
\end{array}\right),
$$

that leads to the disentanglement relations

$$
\begin{aligned}
S(\xi, 0, \zeta) & =\exp \left(\xi K_{-}-\zeta K_{+}\right) \\
& =\exp \left(-\zeta \frac{\operatorname{th}(\sqrt{\xi \zeta})}{\sqrt{\xi \zeta}} K_{+}\right) \exp \left(\xi \frac{\operatorname{sh}(2 \sqrt{\xi \zeta})}{2 \sqrt{\xi \zeta}} K_{-}\right)(\operatorname{ch}(\sqrt{\xi \zeta}))^{-2 K_{0}} \\
& =\exp \left(-\zeta \frac{\operatorname{th}(\sqrt{\xi \zeta})}{\sqrt{\xi \zeta}} K_{+}\right)(\operatorname{ch}(\sqrt{\xi \zeta}))^{-2 K_{0}} \exp \left(\xi \frac{\operatorname{th}(\sqrt{\xi \zeta})}{\sqrt{\xi \zeta}} K_{-}\right) \\
& =(\operatorname{ch}(\sqrt{\xi \zeta}))^{-2 K_{0}} \exp \left(-\zeta \frac{\operatorname{sh}(2 \sqrt{\xi \zeta})}{2 \sqrt{\xi \zeta}} K_{+}\right) \exp \left(\xi \frac{\operatorname{th}(\sqrt{\xi \zeta})}{\sqrt{\xi \zeta}} K_{-}\right) \\
& =(\operatorname{ch}(\sqrt{\xi \zeta}))^{2 K_{0}} \exp \left(\xi \frac{\operatorname{sh}(2 \sqrt{\xi \zeta})}{2 \sqrt{\xi \zeta}} K_{-}\right) \exp \left(-\zeta \frac{\operatorname{th}(\sqrt{\xi \zeta})}{\sqrt{\xi \zeta}} K_{+}\right) \\
& =\exp \left(\xi \frac{\operatorname{th}(\sqrt{\xi \zeta})}{\sqrt{\xi \zeta}} K_{-}\right)(\operatorname{ch}(\sqrt{\xi \zeta}))^{2 K_{0}} \exp \left(-\zeta \frac{\operatorname{th}(\sqrt{\xi \zeta})}{\sqrt{\xi \zeta}} K_{+}\right) \\
& =\exp \left(\xi \frac{\operatorname{th}(\sqrt{\xi \zeta})}{\sqrt{\xi \zeta}} K_{-}\right) \exp \left(-\zeta \frac{\operatorname{sh}(2 \sqrt{\xi \zeta})}{2 \sqrt{\xi \zeta}} K_{+}\right)(\operatorname{ch}(\sqrt{\xi \zeta}))^{2 K_{0}},
\end{aligned}
$$


where again we may insert the realization (A.4). In addition to the operators $K_{-}$and $K_{+}$we have involved then on the right-hand side also the operator $K_{0}$.

If we make in (A.15) the transition to the variables in the non-unitary approach

$$
S(\xi, 0, \zeta) \rightarrow S\left(\frac{\operatorname{Arth}(\sqrt{\xi \zeta})}{\sqrt{\xi \zeta}} \xi, 0, \frac{\operatorname{Arth}(\sqrt{\xi \zeta})}{\sqrt{\xi \zeta}} \zeta\right),
$$

then the matrix in (A.15) makes the transition to

$$
\left(\begin{array}{ll}
\kappa, & \lambda \\
\mu, & v
\end{array}\right) \rightarrow\left(\begin{array}{cc}
\frac{1}{\sqrt{1-\xi \zeta}} & \frac{\xi}{\sqrt{1-\xi \zeta}} \\
\frac{\zeta}{\sqrt{1-\xi \zeta}} & \frac{1}{\sqrt{1-\xi \zeta}}
\end{array}\right),
$$

as it is easily to see.

The next considerations are the effort to split the general operator $S(\xi, \eta, \zeta)$ into products of a proper squeezing operator $S\left(\xi^{\prime}, 0, \zeta^{\prime}\right)$ and a proper rotation operator $S\left(0, \eta^{\prime}, 0\right)$. The general two-dimensional matrix (A.7) can be split also in the following two ways

$$
\begin{aligned}
\left(\begin{array}{ll}
\kappa, & \lambda \\
\mu, & v
\end{array}\right) & =\left(\begin{array}{cc}
\sqrt{\kappa \nu} & \lambda \sqrt{\frac{\kappa}{v}} \\
\mu \sqrt{\frac{v}{\kappa}} & \sqrt{\kappa v}
\end{array}\right)\left(\begin{array}{cc}
\sqrt{\frac{\kappa}{v}} & 0 \\
0 & \sqrt{\frac{v}{\kappa}}
\end{array}\right) \\
& =\left(\begin{array}{cc}
\sqrt{\frac{\kappa}{v}} & 0 \\
0 & \sqrt{\frac{v}{\kappa}}
\end{array}\right)\left(\begin{array}{cc}
\sqrt{\kappa \nu} & \lambda \sqrt{\frac{v}{\kappa}} \\
\mu \sqrt{\frac{\kappa}{v}} & \sqrt{\kappa v}
\end{array}\right) .
\end{aligned}
$$

This corresponds explicitly to the two possibilities $\left(\varepsilon \equiv \sqrt{\xi \zeta-\eta^{2}}\right)$

$$
\begin{aligned}
& =\left(\begin{array}{ll}
\kappa & \lambda \\
\mu & v
\end{array}\right)=\left(\begin{array}{cc}
\sqrt{\operatorname{ch}^{2}(\varepsilon)+\eta^{2} \frac{\operatorname{sh}^{2}(\varepsilon)}{\varepsilon^{2}}} & \xi \frac{\operatorname{sh}(\varepsilon)}{\varepsilon} \sqrt{\frac{\operatorname{ch}(\varepsilon)-\mathrm{i} \eta \frac{\operatorname{sh}(\varepsilon)}{\varepsilon}}{\operatorname{ch}(\varepsilon)+\mathrm{i} \eta \frac{\operatorname{sh}(\varepsilon)}{\varepsilon}}} \\
\zeta \frac{\operatorname{sh}(\varepsilon)}{\varepsilon} \sqrt{\frac{\operatorname{ch}(\varepsilon)+\mathrm{i} \eta \frac{\operatorname{sh}(\varepsilon)}{\varepsilon}}{\operatorname{ch}(\varepsilon)-\mathrm{i} \eta \frac{\operatorname{sh}(\varepsilon)}{\varepsilon}}} & \sqrt{\operatorname{ch}^{2}(\varepsilon)+\eta^{2} \frac{\operatorname{sh}^{2}(\varepsilon)}{\varepsilon^{2}}}
\end{array}\right) \\
& \times\left(\begin{array}{cc}
\sqrt{\frac{\operatorname{ch}(\varepsilon)-\mathrm{i} \eta \frac{\operatorname{sh}(\varepsilon)}{\varepsilon}}{\operatorname{ch}(\varepsilon)+\mathrm{i} \eta \frac{\operatorname{sh}(\varepsilon)}{\varepsilon}}} & 0 \\
0 & \sqrt{\frac{\operatorname{ch}(\varepsilon)+\mathrm{i} \eta \frac{\operatorname{sh}(\varepsilon)}{\varepsilon}}{\operatorname{ch}(\varepsilon)-\mathrm{i} \eta \frac{\operatorname{sh}(\varepsilon)}{\varepsilon}}}
\end{array}\right)
\end{aligned}
$$




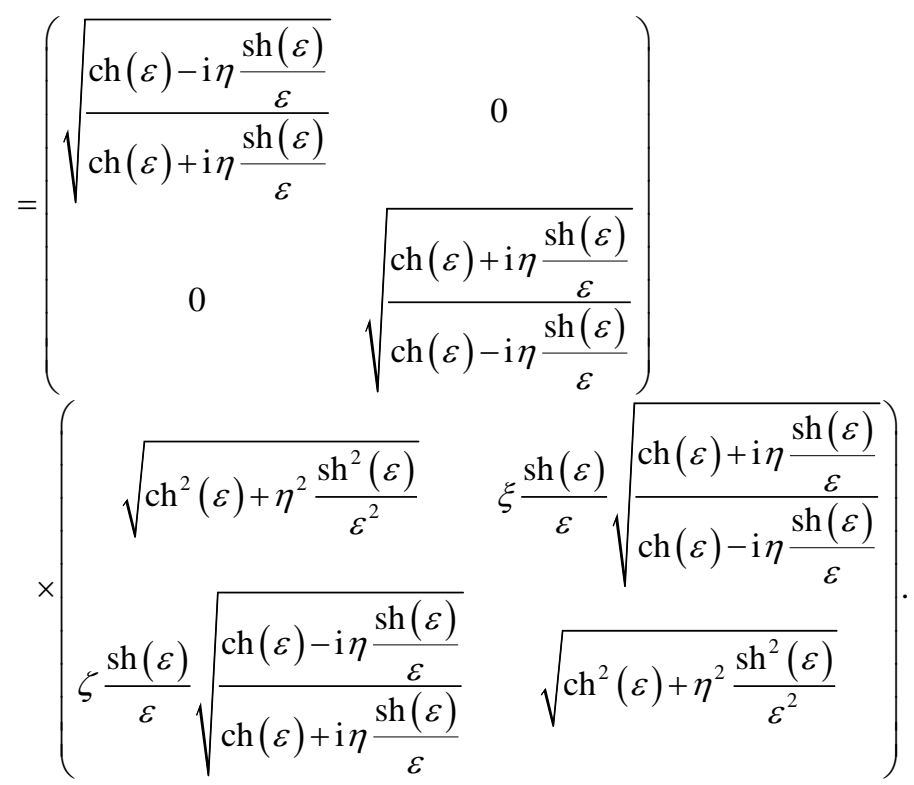

and if we introduce abbreviations

$$
\begin{aligned}
\left(\begin{array}{ll}
\kappa & \lambda \\
\mu & v
\end{array}\right) & =\left(\begin{array}{cc}
\operatorname{ch}\left(\sqrt{\xi^{\prime} \zeta^{\prime}}\right) & \xi^{\prime} \frac{\operatorname{sh}\left(\sqrt{\zeta^{\prime} \zeta^{\prime}}\right)}{\sqrt{\xi^{\prime} \zeta^{\prime}}} \\
\zeta^{\prime} \frac{\operatorname{sh}\left(\sqrt{\zeta^{\prime} \zeta^{\prime}}\right)}{\sqrt{\xi^{\prime} \zeta^{\prime}}} & \operatorname{ch}\left(\sqrt{\xi^{\prime} \zeta^{\prime}}\right)
\end{array}\right)\left(\begin{array}{cc}
\mathrm{e}^{-\mathrm{i} \eta_{0}} & 0 \\
0 & \mathrm{e}^{\mathrm{i} \eta_{0}}
\end{array}\right) \\
& =\left(\begin{array}{cc}
\mathrm{e}^{-\mathrm{i} \eta_{0}} & 0 \\
0 & \mathrm{e}^{\mathrm{i} \eta_{0}}
\end{array}\right)\left(\begin{array}{cc}
\operatorname{ch}\left(\sqrt{\xi^{\prime \prime} \zeta^{\prime \prime}}\right) & \xi^{\prime \prime} \frac{\operatorname{sh}\left(\sqrt{\zeta^{\prime \prime} \zeta^{\prime \prime}}\right)}{\sqrt{\xi^{\prime \prime} \zeta^{\prime \prime}}} \\
\zeta^{\prime \prime} \frac{\operatorname{sh}\left(\sqrt{\zeta^{\prime \prime} \zeta^{\prime \prime}}\right)}{\sqrt{\xi^{\prime \prime} \zeta^{\prime \prime}}} & \operatorname{ch}\left(\sqrt{\xi^{\prime \prime} \zeta^{\prime \prime}}\right)
\end{array}\right) .
\end{aligned}
$$

The rotation operators are the same in both cases and the squeezing operators distinguish themselves only by phase factors in the non-diagonal matrix elements. We have

$$
\left(\begin{array}{cc}
\mathrm{e}^{-\mathrm{i} \eta_{0}} & 0 \\
0 & \mathrm{e}^{\mathrm{i} \eta_{0}}
\end{array}\right), \Leftrightarrow S\left(0, \eta_{0}, 0\right)=\mathrm{e}^{\mathrm{i} \frac{\eta_{0}}{2}\left(a a^{\dagger}+a^{\dagger} a\right)} .
$$

The first possibility of the splitting in a squeezing and a rotation part is

$$
\begin{aligned}
\left(\begin{array}{ll}
\kappa & \lambda \\
\mu & v
\end{array}\right) & =\left(\begin{array}{cc}
\operatorname{ch}(\varepsilon)-\mathrm{i} \eta \frac{\operatorname{sh}(\varepsilon)}{\varepsilon} & \xi \frac{\operatorname{sh}(\varepsilon)}{\varepsilon} \\
\zeta \frac{\operatorname{sh}(\varepsilon)}{\varepsilon} & \operatorname{ch}(\varepsilon)+\mathrm{i} \eta \frac{\operatorname{sh}(\varepsilon)}{\varepsilon}
\end{array}\right) \\
& =\left(\begin{array}{cc}
\sqrt{\operatorname{ch}^{2}(\varepsilon)+\eta^{2} \frac{\operatorname{sh}^{2}(\varepsilon)}{\varepsilon^{2}}} & \xi \frac{\operatorname{sh}(\varepsilon)}{\varepsilon} \sqrt{\frac{\operatorname{ch}(\varepsilon)-\mathrm{i} \eta \frac{\operatorname{sh}(\varepsilon)}{\varepsilon}}{\operatorname{ch}(\varepsilon)+\mathrm{i} \eta \frac{\operatorname{sh}(\varepsilon)}{\varepsilon}}} \\
\zeta \frac{\operatorname{sh}(\varepsilon)}{\varepsilon} \sqrt{\frac{\operatorname{ch}(\varepsilon)+\mathrm{i} \eta \frac{\operatorname{sh}(\varepsilon)}{\varepsilon}}{\operatorname{ch}(\varepsilon)-\mathrm{i} \eta \frac{\operatorname{sh}(\varepsilon)}{\varepsilon}}} & \sqrt{\operatorname{ch}^{2}(\varepsilon)+\eta^{2} \frac{\operatorname{sh}^{2}(\varepsilon)}{\varepsilon^{2}}}
\end{array}\right)
\end{aligned}
$$




$$
\begin{aligned}
& \times\left(\begin{array}{cc}
\sqrt{\frac{\operatorname{ch}(\varepsilon)-\mathrm{i} \eta \frac{\operatorname{sh}(\varepsilon)}{\varepsilon}}{\operatorname{ch}(\varepsilon)+\mathrm{i} \eta \frac{\operatorname{sh}(\varepsilon)}{\varepsilon}}} & 0 \\
0 & \sqrt{\frac{\operatorname{ch}(\varepsilon)+\mathrm{i} \eta \frac{\operatorname{sh}(\varepsilon)}{\varepsilon}}{\operatorname{ch}(\varepsilon)-\mathrm{i} \eta \frac{\operatorname{sh}(\varepsilon)}{\varepsilon}}}
\end{array}\right) \\
& =\left(\begin{array}{cc}
\operatorname{ch}\left(\sqrt{\xi^{\prime} \zeta^{\prime}}\right), & \xi^{\prime} \frac{\operatorname{sh}\left(\sqrt{\zeta^{\prime} \zeta^{\prime}}\right)}{\sqrt{\xi^{\prime} \zeta^{\prime}}} \\
\zeta^{\prime} \frac{\operatorname{sh}\left(\sqrt{\zeta^{\prime} \zeta^{\prime}}\right)}{\sqrt{\xi^{\prime} \zeta^{\prime}}}, & \operatorname{ch}\left(\sqrt{\xi^{\prime} \zeta^{\prime}}\right)
\end{array}\right)\left(\begin{array}{cc}
\mathrm{e}^{-i} \eta_{0}, & 0 \\
0, & \mathrm{e}^{\mathrm{i} \eta_{0}}
\end{array}\right) \\
& =\left(\begin{array}{cc}
\sqrt{\kappa \nu} & \sqrt{\frac{\kappa}{v}} \lambda \\
\sqrt{\frac{\nu}{\kappa}} \mu & \sqrt{\kappa \nu}
\end{array}\right)\left(\begin{array}{cc}
\sqrt{\frac{\kappa}{v}} & 0 \\
0 & \sqrt{\frac{\nu}{\kappa}}
\end{array}\right),
\end{aligned}
$$

with correspondences

$$
\begin{aligned}
& \operatorname{ch}\left(\sqrt{\xi^{\prime} \zeta^{\prime}}\right)=\sqrt{\operatorname{ch}^{2}(\varepsilon)+\eta^{2} \frac{\operatorname{sh}^{2}(\varepsilon)}{\varepsilon^{2}}}=\sqrt{1+\xi \zeta \frac{\operatorname{sh}^{2}(\varepsilon)}{\varepsilon^{2}}}, \\
& \operatorname{sh}\left(\sqrt{\xi^{\prime} \zeta^{\prime}}\right)=\sqrt{\xi \zeta} \frac{\operatorname{sh}(\varepsilon)}{\varepsilon}, \operatorname{th}\left(\sqrt{\xi^{\prime} \zeta^{\prime}}\right)=\frac{\sqrt{\xi \zeta} \operatorname{sh}(\varepsilon)}{\sqrt{1+\xi \zeta \operatorname{sh}^{2}(\varepsilon)}}, \\
& \frac{\operatorname{sh}\left(\sqrt{\xi^{\prime} \zeta^{\prime}}\right)}{\sqrt{\xi^{\prime} \zeta^{\prime}}}=\frac{\sqrt{\xi \zeta} \frac{\operatorname{sh}(\varepsilon)}{\varepsilon}}{\operatorname{Arsh}\left(\sqrt{\xi \zeta} \frac{\operatorname{sh}(\varepsilon)}{\varepsilon}\right)}, \\
& \mathrm{e}^{\mathrm{i} \mathrm{i} \eta_{0}}=\sqrt{\frac{\operatorname{ch}(\varepsilon) \pm \mathrm{i} \eta \frac{\operatorname{sh}(\varepsilon)}{\varepsilon}}{\operatorname{ch}(\varepsilon) \mp \mathrm{i} \eta \frac{\operatorname{sh}(\varepsilon)}{\varepsilon}}}=\frac{\operatorname{ch}(\varepsilon) \pm \mathrm{i} \eta \frac{\operatorname{sh}(\varepsilon)}{\varepsilon}}{\sqrt{\operatorname{ch}^{2}(\varepsilon)+\eta^{2} \frac{\operatorname{sh}^{2}(\varepsilon)}{\varepsilon^{2}}}}=\frac{\sqrt{\operatorname{ch}^{2}(\varepsilon)+\eta^{2} \frac{\operatorname{sh}^{2}(\varepsilon)}{\varepsilon^{2}}}}{\operatorname{ch}(\varepsilon) \mp \mathrm{i} \eta \frac{\operatorname{sh}(\varepsilon)}{\varepsilon}}, \\
& \operatorname{tg}\left(\eta_{0}\right)=\eta \frac{\operatorname{th}(\varepsilon)}{\varepsilon}, \quad \cos \left(\eta_{0}\right)=\frac{\operatorname{ch}(\varepsilon)}{\sqrt{\operatorname{ch}^{2}(\varepsilon)+\eta^{2} \frac{\operatorname{sh}^{2}(\varepsilon)}{\varepsilon^{2}}}}, \\
& \sin \left(\eta_{0}\right)=\frac{\eta \frac{\operatorname{sh}(\varepsilon)}{\varepsilon}}{\sqrt{\operatorname{ch}^{2}(\varepsilon)+\eta^{2} \frac{\operatorname{sh}^{2}(\varepsilon)}{\varepsilon^{2}}}} \\
& \xi^{\prime}=\frac{\operatorname{Arsh}\left(\sqrt{\xi \zeta} \frac{\operatorname{sh}(\varepsilon)}{\varepsilon}\right)}{\sqrt{\xi \zeta} \frac{\operatorname{sh}(\varepsilon)}{\varepsilon}} \xi \mathrm{e}^{-\mathrm{i} \eta}, \quad \zeta^{\prime}=\frac{\operatorname{Arsh}\left(\sqrt{\xi \zeta} \frac{\operatorname{sh}(\varepsilon)}{\varepsilon}\right)}{\sqrt{\xi \zeta} \frac{\operatorname{sh}(\varepsilon)}{\varepsilon}} \zeta \mathrm{e}^{\mathrm{i} \eta} .
\end{aligned}
$$


The parameters in the factorized matrices are $(\kappa, \lambda, \mu, v$ are such as in (A.7)) $S(\xi, \eta, \zeta)$

$$
\begin{aligned}
= & S\left(\frac{\operatorname{Arsh}(\sqrt{\lambda \mu})}{\sqrt{\lambda \mu}} \sqrt{\frac{\kappa}{v}} \lambda, 0, \frac{\operatorname{Arsh}(\sqrt{\lambda \mu})}{\sqrt{\lambda \mu}} \sqrt{\frac{\nu}{\kappa}} \mu\right) S\left(0, \mathrm{iArsh}\left(\frac{1}{2}\left(\sqrt{\frac{\kappa}{v}}-\sqrt{\frac{v}{\kappa}}\right)\right), 0\right) \\
= & S\left(\frac{\operatorname{Arsh}\left(\sqrt{\xi \zeta} \frac{\operatorname{sh}(\varepsilon)}{\varepsilon}\right)}{\sqrt{\xi \zeta}} \frac{\operatorname{ch}(\varepsilon)-\mathrm{i} \eta \frac{\operatorname{sh}(\varepsilon)}{\varepsilon}}{\sqrt{\operatorname{ch}^{2}(\varepsilon)+\eta^{2} \frac{\operatorname{sh}^{2}(\varepsilon)}{\varepsilon^{2}}}} \xi, 0,\right. \\
& \left.\frac{\operatorname{Arsh}\left(\sqrt{\xi \zeta} \frac{\operatorname{sh}(\varepsilon)}{\varepsilon}\right)}{\sqrt{\xi \zeta}} \frac{\operatorname{ch}(\varepsilon)+\mathrm{i} \eta \frac{\operatorname{sh}(\varepsilon)}{\varepsilon}}{\sqrt{\operatorname{ch}^{2}(\varepsilon)+\eta^{2} \frac{\operatorname{sh}^{2}(\varepsilon)}{\varepsilon^{2}}}} \zeta\right) \\
& \cdot S\left(0, \mathrm{i} \operatorname{Arsh}\left(-\frac{\mathrm{i} \eta \operatorname{sh}^{2}(\varepsilon)}{\left.\left.\sqrt{\varepsilon^{2}+\xi \zeta \operatorname{sh}^{2}(\varepsilon)}\right), 0\right) .}\right.\right.
\end{aligned}
$$

The second possibility with interchanged order of the splitting in a squeezing and a rotation part is

$$
\begin{aligned}
& \left(\begin{array}{ll}
\kappa & \lambda \\
\mu & v
\end{array}\right)=\left(\begin{array}{cc}
\operatorname{ch}(\varepsilon)-\mathrm{i} \eta \frac{\operatorname{sh}(\varepsilon)}{\varepsilon} & \xi \frac{\operatorname{sh}(\varepsilon)}{\varepsilon} \\
\zeta \frac{\operatorname{sh}(\varepsilon)}{\varepsilon} & \operatorname{ch}(\varepsilon)+\mathrm{i} \eta \frac{\operatorname{sh}(\varepsilon)}{\varepsilon}
\end{array}\right) \\
& =\left(\begin{array}{cc}
\sqrt{\frac{\operatorname{ch}(\varepsilon)-\mathrm{i} \eta \frac{\operatorname{sh}(\varepsilon)}{\varepsilon}}{\operatorname{ch}(\varepsilon)+\mathrm{i} \eta \frac{\operatorname{sh}(\varepsilon)}{\varepsilon}}} & 0 \\
0 & \sqrt{\frac{\operatorname{ch}(\varepsilon)+\mathrm{i} \eta \frac{\operatorname{sh}(\varepsilon)}{\varepsilon}}{\operatorname{ch}(\varepsilon)-\mathrm{i} \eta \frac{\operatorname{sh}(\varepsilon)}{\varepsilon}}}
\end{array}\right) \\
& \times\left(\begin{array}{cc}
\sqrt{\operatorname{ch}^{2}(\varepsilon)+\eta^{2} \frac{\operatorname{sh}^{2}(\varepsilon)}{\varepsilon^{2}}} & \xi \frac{\operatorname{sh}(\varepsilon)}{\varepsilon} \sqrt{\frac{\operatorname{ch}(\varepsilon)+\mathrm{i} \eta \frac{\operatorname{sh}(\varepsilon)}{\varepsilon}}{\operatorname{ch}(\varepsilon)-\mathrm{i} \eta \frac{\operatorname{sh}(\varepsilon)}{\varepsilon}}} \\
\zeta \frac{\operatorname{sh}(\varepsilon)}{\varepsilon} \sqrt{\frac{\operatorname{ch}(\varepsilon)-\mathrm{i} \eta \frac{\operatorname{sh}(\varepsilon)}{\varepsilon}}{\operatorname{ch}(\varepsilon)+\mathrm{i} \eta \frac{\operatorname{sh}(\varepsilon)}{\varepsilon}}} & \sqrt{\operatorname{ch}^{2}(\varepsilon)+\eta^{2} \frac{\operatorname{sh}^{2}(\varepsilon)}{\varepsilon^{2}}}
\end{array}\right) \\
& =\left(\begin{array}{cc}
\mathrm{e}^{-\mathrm{i} \eta_{0}} & 0 \\
0 & \mathrm{e}^{\mathrm{i} \eta_{0}}
\end{array}\right)\left(\begin{array}{cc}
\operatorname{ch}\left(\sqrt{\xi^{\prime \prime} \zeta^{\prime \prime}}\right) & \xi^{\prime \prime} \frac{\operatorname{sh}\left(\sqrt{\zeta^{\prime \prime} \zeta^{\prime \prime}}\right)}{\sqrt{\xi^{\prime \prime} \zeta^{\prime \prime}}} \\
\zeta^{\prime \prime} \frac{\operatorname{sh}\left(\sqrt{\zeta^{\prime \prime} \zeta^{\prime \prime}}\right)}{\sqrt{\xi^{\prime \prime} \zeta^{\prime \prime}}} & \operatorname{ch}\left(\sqrt{\xi^{\prime \prime} \zeta^{\prime \prime}}\right)
\end{array}\right)
\end{aligned}
$$




$$
=\left(\begin{array}{cc}
\sqrt{\frac{\kappa}{v}} & 0 \\
0 & \sqrt{\frac{v}{\kappa}}
\end{array}\right)\left(\begin{array}{cc}
\sqrt{\kappa v} & \sqrt{\frac{v}{\kappa}} \lambda \\
\sqrt{\frac{\kappa}{v}} \mu & \sqrt{\kappa v}
\end{array}\right) .
$$

The diagonal matrix of a rotation with parameter $\eta_{0}$ is the stable part in the two factorizations (A.23) and (A.26). These considerations show that without disadvantage for the generality we may use the special squeezing operators $S\left(\xi^{\prime}, 0, \zeta^{\prime}\right)$ which are equivalent to the general operators $S(\xi, \eta, \zeta)$ after splitting rotation factors. However, these special squeezing operators alone do not form a group.

\section{Appendix B}

\section{Influence of Displacement and Squeezing of States onto the Wigner Quasi-Probability}

The displacement of states and the squeezing make transformations of the variables in the Wigner quasiprobability which can be given in a general form. For the derivation we use the representation of the Wigner quasiprobability by complex variables $\left(\alpha, \alpha^{*}\right)$ in the following form [22] [29] which is equivalent to the definition given by Wigner [27] [28]

$$
W\left(\alpha, \alpha^{*}\right)=\left\langle\rho \exp \left(-a \frac{\partial}{\partial \alpha}-a^{\dagger} \frac{\partial}{\partial \alpha^{*}}\right)\right\rangle \delta\left(\alpha, \alpha^{*}\right),
$$

where $\rho$ is the density operator of the state. First we investigate the displacement of a state with the density operator $\rho_{0}$ and the Wigner quasiprobability $W_{0}\left(\alpha, \alpha^{*}\right)$ according to

$$
\rho=D\left(\beta, \beta^{*}\right) \rho_{0}\left(D\left(\beta, \beta^{*}\right)\right)^{\dagger} .
$$

Then one finds for $W\left(\alpha, \alpha^{*}\right)$

$$
\begin{aligned}
W\left(\alpha, \alpha^{*}\right) & =\left\langle D\left(\beta, \beta^{*}\right) \rho_{0}\left(D\left(\beta, \beta^{*}\right)\right)^{\dagger} \exp \left(-a \frac{\partial}{\partial \alpha}-a^{\dagger} \frac{\partial}{\partial \alpha^{*}}\right)\right\rangle \delta\left(\alpha, \alpha^{*}\right) \\
& =\left\langle\rho_{0}\left(D\left(\beta, \beta^{*}\right)\right)^{\dagger} \exp \left(-a \frac{\partial}{\partial \alpha}-a^{\dagger} \frac{\partial}{\partial \alpha^{*}}\right) D\left(\beta, \beta^{*}\right)\right\rangle \delta\left(\alpha, \alpha^{*}\right) \\
& =\left\langle\rho_{0}\left(D\left(\beta, \beta^{*}\right)\right)^{\dagger} \exp \left(-(a+\beta I) \frac{\partial}{\partial \alpha}-\left(a^{\dagger}+\beta^{*} I\right) \frac{\partial}{\partial \alpha^{*}}\right)\right\rangle \delta\left(\alpha, \alpha^{*}\right) \\
& =\left\langle\rho_{0} \exp \left(-a \frac{\partial}{\partial \alpha}-a^{\dagger} \frac{\partial}{\partial \alpha^{*}}\right)\right\rangle \exp \left(-\beta \frac{\partial}{\partial \alpha}-\beta^{*} \frac{\partial}{\partial \alpha^{*}}\right) \delta\left(\alpha, \alpha^{*}\right) \\
& =\left\langle\rho_{0} \exp \left(-a \frac{\partial}{\partial \alpha}-a^{\dagger} \frac{\partial}{\partial \alpha^{*}}\right)\right\rangle \delta\left(\alpha-\beta, \alpha^{*}-\beta^{*}\right) .
\end{aligned}
$$

Therefore we obtained

$$
W\left(\alpha, \alpha^{*}\right)=W_{0}\left(\alpha-\beta, \alpha^{*}-\beta^{*}\right) .
$$

This means that the Wigner quasiprobability $W\left(\alpha, \alpha^{*}\right)$ for the displaced 
state is equal to the Wigner quasiprobability $W_{0}\left(\alpha, \alpha^{*}\right)$ for the undisplaced state with displaced arguments corresponding to the displacement parameters $\left(\beta, \beta^{*}\right)$. This displacement property is one of the minimal requirements for a phase-space function $F\left(\alpha, \alpha^{*}\right)$ in quantum theory to be called a quasiprobability.

In widely analogous way one may treat the squeezing of a state with density operator $\rho_{0}$ according to

$$
\rho=S\left(\zeta^{*}, \eta=\eta^{*}, \zeta\right) \rho_{0}\left(S\left(\zeta^{*}, \eta=\eta^{*}, \zeta\right)\right)^{\dagger}
$$

Instead of the unitary squeezing operators $S\left(\zeta^{*}, \eta=\eta^{*}, \zeta\right)$ we will calculate the transformations first a little more general with the operators $S(\xi, \eta, \zeta)$ and at the end we will specialize the result to $\xi=\zeta^{*}$ and $\eta=\eta^{*}$. Therefore, we have first to substitute $\left(S\left(\zeta^{*}, \eta=\eta^{*}, \zeta\right)\right)^{\dagger} \rightarrow(S(\xi, \eta, \zeta))^{-1}=S(-\xi,-\eta,-\zeta)$ and find

$$
\begin{aligned}
W\left(\alpha, \alpha^{*}\right) & =\left\langle S(\xi, \eta, \zeta) \rho_{0}(S(\xi, \eta, \zeta))^{-1} \exp \left(-a \frac{\partial}{\partial \alpha}-a^{?} \frac{\partial}{\partial \alpha^{*}}\right)\right\rangle \delta(\alpha, \alpha) \\
& =\left\langle\rho_{0}(S(\xi, \eta, \zeta))^{-1} \exp \left(-a \frac{\partial}{\partial \alpha}-a^{\dagger} \frac{\partial}{\partial \alpha^{*}}\right) S(\xi, \eta, \zeta)\right\rangle \delta\left(\alpha, \alpha^{*}\right) \\
& =\left\langle\rho_{0} \exp \left(-\left(v a-\mu a^{\dagger}\right) \frac{\partial}{\partial \alpha}-\left(-\lambda a+\kappa a^{\dagger}\right) \frac{\partial}{\partial \alpha^{*}}\right)\right\rangle \delta\left(\alpha, \alpha^{*}\right) \\
& =\left\langle\rho_{0} \exp \left(-a\left(v \frac{\partial}{\partial \alpha}-\lambda \frac{\partial}{\partial \alpha^{*}}\right)-a^{\dagger}\left(-\mu \frac{\partial}{\partial \alpha}+\kappa \frac{\partial}{\partial \alpha^{*}}\right)\right)\right\rangle \delta\left(\alpha, \alpha^{*}\right) \\
& =\left\langle\rho_{0} \exp \left(-a \frac{\partial}{\partial \gamma}-a^{\dagger} \frac{\partial}{\partial \gamma^{\prime}}\right)\right\rangle \delta\left(v \gamma-\mu \gamma^{\prime},-\lambda \gamma+\kappa \gamma^{\prime}\right) \\
& =\left\langle\rho_{0} \exp \left(-a \frac{\partial}{\partial \gamma}-a^{\dagger} \frac{\partial}{\partial \gamma^{\prime}}\right)\right\rangle \delta\left(\gamma, \gamma^{\prime}\right) .
\end{aligned}
$$

Formally this can be written

$$
W\left(\alpha, \alpha^{*}\right) \equiv W_{0}\left(\gamma, \gamma^{\prime}\right)
$$

with the matrix elements in explicit form given in (A.7). This corresponds to the transformation

$$
\begin{aligned}
& \gamma=\kappa \alpha+\mu \alpha^{*}, \quad \alpha=v \gamma-\mu \gamma^{\prime}, \quad \frac{\partial}{\partial \gamma}=v \frac{\partial}{\partial \alpha}-\lambda \frac{\partial}{\partial \alpha^{*}}, \\
& \gamma^{\prime}=\lambda \alpha+v \alpha^{*}, \quad \alpha^{*}=-\lambda \gamma+\kappa \gamma^{\prime}, \quad \frac{\partial}{\partial \gamma^{\prime}}=-\mu \frac{\partial}{\partial \alpha}+\kappa \frac{\partial}{\partial \alpha^{*}} .
\end{aligned}
$$

In the last step in (B.6) was used that the two-dimensional delta function $\delta\left(\gamma, \gamma^{\prime}\right)$ is invariant with respect to a (complex) unimodular transformation of the variables. This can be proved, for example, by transition to real variables and transformation to principal axes (see below).

In the special case of unitary transformations with the operator $S\left(\zeta^{*}, \eta=\eta^{*}, \zeta\right)$ we can substitute $\kappa \rightarrow v^{*}, \lambda \rightarrow \mu^{*}$ and $\gamma^{\prime} \rightarrow \gamma^{*}$ and (B.7) 
using (B.8) can be specialized to [35]

$$
W\left(\alpha, \alpha^{*}\right)=W_{0}\left(v^{*} \alpha+\mu \alpha^{*}, \mu^{*} \alpha+v \alpha^{*}\right) \equiv W_{0}\left(\alpha^{\prime}, \alpha^{\prime *}\right) .
$$

We mention that a displacement relation of the form (B.4) is true for all quasiprobabilities (e.g., $Q\left(\alpha, \alpha^{*}\right)$ and $P\left(\alpha, \alpha^{*}\right)$ ) whereas for squeezing of states the form (B.9) is only true for the Wigner quasiprobability. The reason is that we do not have in other cases the operator $-a \frac{\partial}{\partial \alpha}-a^{\dagger} \frac{\partial}{\partial \alpha^{*}}$ as a whole in the exponent of only one exponential function.

The transformation of the variables $\left(\alpha^{\prime}, \alpha^{\prime *}\right) \leftrightarrow\left(\alpha, \alpha^{*}\right)$ possesses the property

$$
\begin{aligned}
\mathrm{d} \alpha \wedge \mathrm{d} \alpha^{*} & =\left(v^{*} \mathrm{~d} \alpha^{\prime}+\mu \mathrm{d} \alpha^{\prime *}\right) \wedge\left(\mu^{*} \mathrm{~d} \alpha+v \mathrm{~d} \alpha^{\prime *}\right) \\
& =\left(v v^{*}-\mu \mu^{*}\right) \mathrm{d} \alpha^{\prime} \wedge \mathrm{d} \alpha^{\prime *}=\mathrm{d} \alpha^{\prime} \wedge \mathrm{d} \alpha^{\prime *}
\end{aligned}
$$

and therefore

$$
\frac{\mathrm{d} q \wedge \mathrm{d} p}{2 \hbar}=\frac{\mathrm{i}}{2} \mathrm{~d} \alpha \wedge \mathrm{d} \alpha^{*}=\frac{\mathrm{i}}{2} \mathrm{~d} \alpha^{\prime} \wedge \mathrm{d} \alpha^{\prime *}=\frac{\mathrm{d} q^{\prime} \wedge \mathrm{d} p^{\prime}}{2 \hbar} .
$$

This means that each area element of the Wigner quasiprobability $W_{0}\left(\alpha, \alpha^{*}\right)$ is mapped in a new area element of $W\left(\alpha, \alpha^{*}\right)$ of the same area with preservation of the topology but without preservation of angles such as for transformations in classical mechanics. Therefore, for example, if the Wigner quasiprobability possesses regions of negativity (squeezed coherent states do not possess such regions) then after the transformation the area of negativity remains the same as before the transformation. The similar property is true for displacements and is here obvious.

A simple proof of the invariance of the two-dimensional delta function under (in general, complex) unimodular transformations of the variables by a matrix $\left(\begin{array}{ll}a & b \\ c & d\end{array}\right)$ with $a d-b c=1$ can be given by extension of coordinates $(x, y)$ to their own complex planes according to

$$
\begin{aligned}
& \delta(a x+b y) \delta(c x+d y) \\
& =\delta\left(\frac{(a d-b c)}{d} x\right) \delta\left(d\left(\frac{c}{d} x+y\right)\right)=\delta\left(\frac{x}{d}\right) \delta\left(d\left(\frac{c}{d} x+y\right)\right) \\
& =\delta\left(\frac{x}{d}\right) \delta(d y)=\delta(x) \delta(y), \quad(a d-b c=1) .
\end{aligned}
$$

This is then also correct for the representation of the two-dimensional delta function by complex variables.

\section{Appendix C}

\section{An Identity between Jacobi Polynomials with Different Arguments}

We derive here an identity between Jacobi polynomials which we used in Section 5 in specialized form. 
Starting from the right-hand side of (5.1) we make a transformation of the following more general expression

$$
\begin{aligned}
& \left(1-y^{2}\right)^{\alpha+\frac{1}{2}} \frac{\partial^{n}}{\partial y^{n}} \frac{1}{\left(1-y^{2}\right)^{\alpha+\frac{1}{2}}} \\
& =\left(\frac{\partial}{\partial y}+\frac{(2 \alpha+1) y}{1-y^{2}}\right)^{n} \\
& =\left(\frac{\partial}{\partial y}-\frac{\alpha+\frac{1}{2}}{1-y}-\frac{\alpha+\frac{1}{2}}{1+y}\right)^{n} \\
& =\sum_{j=0}^{n}\left(-\frac{2}{1-y^{2}}\right)^{j} \mathrm{P}_{j}^{\left(-\alpha-\frac{1}{2}-j,-\alpha-\frac{1}{2}-j\right)}(y) \frac{n !}{(n-j) !} \frac{\partial^{n-j}}{\partial^{n-j}} .
\end{aligned}
$$

This is an operator identity which in the last line is written in the entangled form derived in [32] and was given there in the following here slightly changed form

$$
\begin{aligned}
& \left(\frac{\partial}{\partial y}-\frac{\alpha^{\prime}}{1-y}+\frac{\beta^{\prime}}{1+y}\right)^{n} \\
& =\sum_{j=0}^{j}\left(-\frac{2}{1-y^{2}}\right)^{j} \mathrm{P}_{j}^{\left(\alpha^{\prime}-j, \beta^{\prime}-j\right)}(y) \frac{n !}{(n-j) !} \frac{\partial^{n-j}}{\partial y^{n-j}},
\end{aligned}
$$

and can be proved by complete induction. With $\mathrm{P}_{n}^{(\alpha, \beta)}(u)$ are denoted the Jacobi polynomials.

The operator identity (C.1) can be applied to arbitrary functions of the variable $y$. If we apply it to the function $f(y)=1$ we find

$$
\begin{aligned}
& \left(1-y^{2}\right)^{\alpha+\frac{1}{2}} \frac{\partial^{n}}{\partial y^{n}} \frac{1}{\left(1-y^{2}\right)^{\alpha+\frac{1}{2}}} 1 \\
& =\sum_{j=0}^{n}\left(-\frac{2}{1-y^{2}}\right)^{j} \mathrm{P}_{j}^{\left(-\alpha-\frac{1}{2}-j,-\alpha-\frac{1}{2}-j\right)}(y) \frac{n !}{(n-j) !} \frac{\partial^{n-j}}{\partial y^{n-j}} 1 \\
& =n !\left(-\frac{2}{1-y^{2}}\right)^{n} \mathrm{P}_{n}^{\left(-\alpha-\frac{1}{2}-n,-\alpha-\frac{1}{2}-n\right)}(y) .
\end{aligned}
$$

Up to this point it may be considered as a transformation of the (Rodrigueskind) definition of specialized Jacobi polynomials given by Szegö [30] (see also [31]).

The special case of Jacobi polynomials with equal upper indices $\mathrm{P}_{n}^{(\beta, \beta)}(y)$ is called Ultraspherical polynomials and they are related to the Gegenbauer polynomials $\mathrm{C}_{n}^{v}(y)$ in the following simple way

$$
\mathrm{P}_{n}^{(\beta, \beta)}(y)=\frac{(2 \beta) !(n+\beta) !}{\beta !(n+2 \beta) !} \mathrm{C}_{n}^{\beta+\frac{1}{2}}(y) .
$$

They possess the following for us interesting expansions (Equations (4.5) and 
(5.2) in [32])

$$
\begin{aligned}
\mathrm{P}_{n}^{(\beta, \beta)}(y) & =\frac{2^{2 \beta}(n+\beta) !}{(n+2 \beta) !} \sum_{k=0}^{\left[\frac{n}{2}\right]} \frac{(-1)^{k}\left(n+\beta-k-\frac{1}{2}\right) !}{k !(n-2 k) !\left(-\frac{1}{2}\right) !}(2 y)^{n-2 k} \\
& =\sum_{j=0}^{\left[\frac{n}{2}\right]} \frac{(-1)^{j}(n+\beta) !}{j !(n-2 j) !(j+\beta) ! 2^{2 j}}\left(1-y^{2}\right)^{j} y^{n-2 j}
\end{aligned}
$$

Substituting in (C.5) $\beta=-\alpha-\frac{1}{2}-n$ and using transformation relations for the factorials one finds the following representation of the Ultraspherical polynomials involved in (C.3)

$$
\begin{aligned}
& \mathrm{P}_{n}^{\left(-\alpha-\frac{1}{2}-n,-\alpha-\frac{1}{2}-n\right)}(y) \\
& =\frac{(-1)^{n} \alpha !(n+2 \alpha) !}{2^{2 n}(2 \alpha) !(n+\alpha) !} \sum_{k=0}^{\left[\frac{n}{2}\right]} \frac{(n+\alpha) !}{k !(n-2 k) !(k+\alpha) !}(2 y)^{n-2 k} .
\end{aligned}
$$

In [32] (Equation (5.4)) it was derived an identity which can be written

$$
\left(2 \sqrt{1+x^{2}}\right)^{n} \mathrm{P}_{n}^{(\alpha, \alpha)}\left(\frac{x}{\sqrt{1+x^{2}}}\right)=\sum_{k=0}^{\left[\frac{n}{2}\right]} \frac{(-1)^{k}(n+\alpha) !}{k !(n-2 k) !(k+\alpha) !}(2 x)^{n-2 k},
$$

or after the substitution $x=\mathrm{i} y$

$$
\begin{aligned}
& \left(-\mathrm{i} 2 \sqrt{1-y^{2}}\right)^{n} \mathrm{P}_{n}^{(\alpha, \alpha)}\left(\frac{\mathrm{i} y}{\sqrt{1-y^{2}}}\right) \\
& =\sum_{k=0}^{\left[\frac{n}{2}\right.} \frac{(n+\alpha) !}{k !(n-2 k) !(k+\alpha) !}(2 y)^{n-2 k} .
\end{aligned}
$$

An identity for these polynomials and their explicit representation is

$$
\begin{aligned}
& \left(-\mathrm{i} 2 \sqrt{1-y^{2}}\right)^{n} \mathrm{P}_{n}^{(\alpha, \alpha)}\left(\frac{\mathrm{i} y}{\sqrt{1-y^{2}}}\right) \\
& =\frac{(-1)^{n} 2^{2 n}(2 \alpha) !(n+\alpha) !}{\alpha !(n+2 \alpha) !} \mathrm{P}_{n}^{\left(-\alpha-\frac{1}{2}-n,-\alpha-\frac{1}{2}-n\right)}(y) .
\end{aligned}
$$

These relations were applied in Section 5 to get the representation of $\overline{a^{\dagger k} a^{k}}$ by known polynomials.

\section{Appendix D}

\section{Generating Functions for Hermite Polynomials}

For easy use we collect here the most important generating functions for Hermite polynomials. The basic generating function for Hermite polynomials is

$$
\sum_{n=0}^{\infty} \frac{t^{n}}{n !} \mathrm{H}_{n}(z)=\exp \left(2 t z-t^{2}\right)
$$


The basic generating function for products of two Hermite polynomials with different arguments is the Mehler formula [31] (chap. 10.13, Equation (22))

$$
\sum_{n=0}^{\infty} \frac{1}{n !}\left(\frac{t}{2}\right)^{n} \mathrm{H}_{n}(x) \mathrm{H}_{n}(y)=\frac{1}{\left(1-t^{2}\right)^{\frac{1}{2}}} \exp \left(\frac{2 t x y-t^{2}\left(x^{2}+y^{2}\right)}{1-t^{2}}\right) .
$$

By setting $y=0$ and using $\mathrm{H}_{2 m}(0)=\frac{(-1)^{m}(2 m) !}{m !}$ and $\mathrm{H}_{2 m+1}(0)=0$ one obtains for even Hermite polynomials and by its differentiation with respect to variable $Z$ for odd Hermite polynomials the following non-trivial generating functions

$$
\begin{aligned}
& \sum_{m=0}^{\infty} \frac{(-1)^{m}}{m !}\left(\frac{t}{2}\right)^{2 m} \mathrm{H}_{2 m}(z)=\frac{1}{\left(1-t^{2}\right)^{\frac{1}{2}}} \exp \left(-\frac{(t z)^{2}}{1-t^{2}}\right), \\
& \sum_{m=0}^{\infty} \frac{(-1)^{m}}{m !}\left(\frac{t}{2}\right)^{2 m+1} \mathrm{H}_{2 m+1}(z)=\frac{t z}{\left(1-t^{2}\right)^{\frac{3}{2}}} \exp \left(-\frac{(t z)^{2}}{1-t^{2}}\right) .
\end{aligned}
$$

The generating functions for even and odd Hermite polynomials which may be easily derived from (D.1) are different from the generating functions (D.3).

\section{Appendix E}

\section{Expectation Values for Squeezed Vacuum States}

We compile here expectation values for ordered operators calculated from the formulae derived in the main text of Section 5.

For a few initial numbers of expectation values $\overline{a^{\dagger k} a^{k}}$ we calculated by (5.2) and (5.3) or from (6.7)

$$
\begin{gathered}
\overline{a^{\dagger 0} a^{0}}=1, \\
\overline{a^{\dagger 1} a^{1}}=\frac{|\zeta|^{2}}{1-|\zeta|^{2}}, \\
\overline{a^{\dagger 2} a^{2}}=\frac{|\zeta|^{2}\left(1+2|\zeta|^{2}\right)}{\left(1-|\zeta|^{2}\right)^{2}}, \\
\overline{a^{\dagger 3} a^{3}}=\frac{3|\zeta|^{4}\left(3+2|\zeta|^{2}\right)}{\left(1-|\zeta|^{2}\right)^{3}}, \\
\overline{a^{\dagger 4} a^{4}}=\frac{3|\zeta|^{4}\left(3+24|\zeta|^{2}+8|\zeta|^{4}\right)}{\left(1-|\zeta|^{2}\right)^{4}}, \\
\overline{a^{\dagger 5} a^{5}}=\frac{15|\zeta|^{6}\left(15+40|\zeta|^{2}+8|\zeta|^{4}\right)}{\left(1-|\zeta|^{2}\right)^{5}}
\end{gathered}
$$


The expectation values of symmetrically (Weyl) ordered operators calculated according to (5.4) are

$$
\begin{gathered}
\overline{\mathcal{S}\left\{a^{\dagger 0} a^{0}\right\}}=1, \\
\overline{\mathcal{S}\left\{a^{\dagger 1} a^{1}\right\}}=\frac{1}{2} \frac{1+|\zeta|^{2}}{1-|\zeta|^{2}}, \\
\overline{\mathcal{S}\left\{a^{\dagger 2} a^{2}\right\}}=\frac{1}{2} \frac{1+4|\zeta|^{2}+|\zeta|^{4}}{\left(1-|\zeta|^{2}\right)^{2}}, \\
\overline{\mathcal{S}\left\{a^{\dagger 3} a^{3}\right\}}=\frac{3}{4} \frac{1+9|\zeta|^{2}+9|\zeta|^{4}+|\zeta|^{6}}{\left(1-|\zeta|^{2}\right)^{3}}, \\
\overline{\mathcal{S}\left\{a^{\dagger 4} a^{4}\right\}}=\frac{3}{2} \frac{1+16|\zeta|^{2}+36|\zeta|^{4}+16|\zeta|^{6}+|\zeta|^{8}}{\left(1-|\zeta|^{2}\right)^{4}} \\
\overline{\mathcal{S}\left\{a^{+5} a^{5}\right\}}=\frac{15}{4} \frac{1+25|\zeta|^{2}+100|\zeta|^{4}+100|\zeta|^{6}+25|\zeta|^{8}+|\zeta|^{10}}{\left(1-|\zeta|^{2}\right)^{5}} .
\end{gathered}
$$

The coefficients in front of powers of $|\zeta|$ in the numerators can be obtained forming the squares of the numbers in the Pascal triangle and, therefore, the polynomials in the numerator are palindromic ones.

For the corresponding expectation values of powers $\overline{N^{l}}$ of the number operator $N$ we found from (5.7)

$$
\begin{aligned}
& \overline{N^{0}}=1 \text {, } \\
& \overline{N^{1}}=\frac{|\zeta|^{2}}{1-|\zeta|^{2}}, \quad \Leftrightarrow|\zeta|^{2}=\frac{\bar{N}}{1+\bar{N}}<1, \\
& \overline{N^{2}}=\frac{|\zeta|^{2}\left(2+|\zeta|^{2}\right)}{\left(1-|\zeta|^{2}\right)^{2}}, \Rightarrow \overline{(\Delta N)^{2}}=\frac{2|\zeta|^{2}}{\left(1-|\zeta|^{2}\right)^{2}}=2 \bar{N}(1+\bar{N}) \text {, } \\
& \overline{N^{3}}=\frac{|\zeta|^{2}\left(4+10|\zeta|^{2}+|\zeta|^{4}\right)}{\left(1-|\zeta|^{2}\right)^{3}} \\
& \Rightarrow \overline{(\Delta N)^{3}}=\frac{4|\zeta|^{2}\left(1+|\zeta|^{2}\right)}{\left(1-|\zeta|^{2}\right)^{3}}=4 \bar{N}(1+\bar{N})(1+2 \bar{N}), \\
& \overline{N^{4}}=\frac{|\zeta|^{2}\left(8+60|\zeta|^{2}+36|\zeta|^{4}+|\zeta|^{6}\right)}{\left(1-|\zeta|^{2}\right)^{4}}, \\
& \Rightarrow \overline{(\Delta N)^{4}}=\frac{4|\zeta|^{2}\left(2+11|\zeta|^{2}+2|\zeta|^{4}\right)}{\left(1-|\zeta|^{2}\right)^{4}} \text {, }
\end{aligned}
$$




$$
\begin{aligned}
\overline{N^{5}} & =\frac{|\zeta|^{2}\left(16+296|\zeta|^{2}+516|\zeta|^{4}+116|\zeta|^{6}+|\zeta|^{8}\right)}{\left(1-|\zeta|^{2}\right)^{5}} \\
& \Rightarrow \overline{(\Delta N)^{5}}=\frac{16|\zeta|^{2}\left(1+16|\zeta|^{2}+16|\zeta|^{4}+|\zeta|^{6}\right)}{\left(1-|\zeta|^{2}\right)^{5}}
\end{aligned}
$$

The photon distribution $p_{n}$ of squeezed vacuum states (2.8) is highly asymmetric to the mean value $\bar{N}$ with a longer tail to higher values of $n$ and, therefore, $\overline{(\Delta N)^{k}}$ is nonnegative for all odd $k$ (in addition to all even $k$ for which this is trivial).

We give yet the initial members of the sequences of expectation values $\overline{a^{2 l}}$ and $\overline{a^{\dagger} a^{2 l-1}}$ for squeezed vacuum states calculated by computer from the more general formulae in Section 6. We found

$$
\begin{aligned}
& \overline{a^{0}}=1, \\
& \overline{a^{2}}=-\frac{\zeta}{1-\zeta \zeta^{*}}, \quad \overline{a^{\dagger} a}=\frac{\zeta^{*} \zeta}{1-\zeta \zeta^{*}}, \\
& \overline{a^{4}}=\frac{3 \zeta^{2}}{\left(1-\zeta \zeta^{*}\right)^{2}}, \quad \overline{a^{\dagger} a^{3}}=-\frac{3 \zeta^{*} \zeta^{2}}{\left(1-\zeta \zeta^{*}\right)^{2}}, \\
& \overline{a^{6}}=-\frac{15 \zeta^{3}}{\left(1-\zeta \zeta^{*}\right)^{3}}, \quad \overline{a^{\dagger} a^{5}}=\frac{15 \zeta^{*} \zeta^{3}}{\left(1-\zeta \zeta^{*}\right)^{3}}, \\
& \overline{a^{8}}=\frac{105 \zeta^{4}}{\left(1-\zeta \zeta^{*}\right)^{4}}, \quad \overline{a^{\dagger} a^{7}}=-\frac{105 \zeta^{*} \zeta^{4}}{\left(1-\zeta \zeta^{*}\right)^{4}}, \\
& \overline{a^{10}}=-\frac{945 \zeta^{5}}{\left(1-\zeta \zeta^{*}\right)^{5}}, \quad \overline{a^{\dagger} a^{9}}=\frac{945 \zeta^{*} \zeta^{5}}{\left(1-\zeta \zeta^{*}\right)^{5}} .
\end{aligned}
$$

The expectation values $\overline{a^{+2 k}}$ and $\overline{a^{+2 k-1} a}$ follow from (E.4) using the general relation $\overline{a^{\dagger k} a^{l}}=\overline{a^{\dagger l} a^{k}}$. The obvious relation seen from (E.4)

$$
\begin{aligned}
& \overline{a^{\dagger} a^{2 l-1}} \equiv\left\langle 0, \zeta\left|a^{\dagger} a^{2 l-1}\right| 0, \zeta\right\rangle=-\zeta^{*}\left\langle 0, \zeta\left|a^{2 l}\right| 0, \zeta\right\rangle \equiv-\zeta^{*} \overline{a^{2 l}}, \\
& \overline{a^{2 l}}=\frac{(-1)^{l}(2 l-1) ! ! \zeta^{l}}{\left(1-\zeta \zeta^{*}\right)^{l}},
\end{aligned}
$$

results from the eigenvalue Equation (2.12) written for the left-hand squeezed vacuum states $\langle 0, \zeta|$ as follows

$$
\langle 0, \zeta|\left(a^{\dagger}+\zeta^{*} a\right)=0 .
$$

We calculated some of the members in (E.4) in independent alternative ways and did not find contradictions.

\section{Notations}

A pair of boson annihilation and creation operators $\left(a, a^{\dagger}\right)$ is connected with 
the Hermitean canonical operators $(Q, P)$ by $\left(\hbar \equiv \frac{h}{2 \pi}\right.$ with $h$ Planck's action quantum)

$$
\begin{aligned}
& \left(a, a^{\dagger}\right)=\left(\frac{Q+\mathrm{i} P}{\sqrt{2 \hbar}}, \frac{Q-\mathrm{i} P}{\sqrt{2 \hbar}}\right), \quad(Q, P)=\sqrt{\frac{\hbar}{2}}\left(a+a^{\dagger},-\mathrm{i}\left(a-a^{\dagger}\right)\right), \\
& {\left[a, a^{\dagger}\right]=I, \quad[Q, P]=\mathrm{i} \hbar I .}
\end{aligned}
$$

Corresponding pairs of complex conjugate variables $\left(\alpha, \alpha^{*}\right)$ and real canonical variables $(q, p)$ are related by

$$
\begin{aligned}
& \left(\alpha, \alpha^{*}\right)=\left(\frac{q+\mathrm{i} p}{\sqrt{2 \hbar}}, \frac{q-\mathrm{i} p}{\sqrt{2 \hbar}}\right), \quad(q, p)=\sqrt{\frac{\hbar}{2}}\left(\alpha+\alpha^{*},-\mathrm{i}\left(\alpha-\alpha^{*}\right)\right), \\
& \frac{\mathrm{i}}{2} \mathrm{~d} \alpha \wedge \mathrm{d} \alpha^{*}=\frac{\mathrm{d} q \wedge \mathrm{d} p}{2 \hbar} .
\end{aligned}
$$

Furthermore: $[A, B]$ denotes the commutator of operators $A$ and $B$ and $\langle A\rangle$ the trace of an operator $A$ (usually in Hilbert space) and $[A]$ the determinant of two-dimensional operators $A$. Expectation values of operators to pure states $|\psi\rangle$ or density operators $\rho$ are denoted by overlining, i.e. $\bar{A} \equiv\langle\psi|A| \psi\rangle=\langle\rho A\rangle$. 